\title{
An Efficient Robust Nonparametric Triple EWMA Wilcoxon Signed-Rank Control Chart for Process Location
}

\author{
Zahid Rasheed $\mathbb{D D}^{1,2}$ Hongying Zhang ${ }^{10},{ }^{1}$ Muhammad Arslan $\left(\mathbb{D},{ }^{3}\right.$ Babar Zaman $\left(\mathbb{D},{ }^{4}\right.$ \\ Syed Masroor Anwar $\left(\mathbb{D},{ }^{5}\right.$ Muhammad Abid $\mathbb{D}^{6},{ }^{6}$ and Saddam Akber Abbasi $\mathbb{D}^{7}$ \\ ${ }^{1}$ School of Mathematics and Statistics, Xi'an Jiaotong University, Xi'an, China \\ ${ }^{2}$ Department of Mathematics, Women University of Azad Jammu and Kashmir, Bagh, AJK, Pakistan \\ ${ }^{3}$ School of Statistics, Shanxi University of Finance and Economics, Taiyuan, China \\ ${ }^{4}$ Department of Mathematics, College of Science, University of Hafr Al Batin, Hafr Al Batin, Saudi Arabia \\ ${ }^{5}$ Department of Statistics, University of Azad Jammu and Kashmir, Muzaffarabad, Pakistan \\ ${ }^{6}$ Department of Statistics, Government College University Faisalabad, Faisalabad, Pakistan \\ ${ }^{7}$ Statistics Program, Department of Mathematics, Statistics and Physics, College of Arts and Science, \\ Qatar University, Doha 2713, Qatar
}

Correspondence should be addressed to Zahid Rasheed; zahidrasheed99@gmail.com

Received 1 July 2021; Accepted 1 October 2021; Published 26 October 2021

Academic Editor: Ishfaq Ahmad

Copyright (C) 2021 Zahid Rasheed et al. This is an open access article distributed under the Creative Commons Attribution License, which permits unrestricted use, distribution, and reproduction in any medium, provided the original work is properly cited.

The nonparametric (NP) control charts are famous for detecting a shift in the process parameters (location and/or dispersion) when the underlying process characteristic does not follow the distributional assumptions. Similarly, when the cost of estimations is very high and the ranking of observational is relatively simple, the ranked set sampling (RSS) technique is preferred over the simple random sampling (SRS) technique. On the other hand, the NP triple exponentially weighted moving average (EWMA) control chart based on SRS is superior to the NP EWMA and NP double EWMA (NP DEWMA) based on the SRS technique to detect a shift in the process location. This study designed an advanced form of NP TEWMA Wilcoxon signed-rank based on RSS, denoted as TEWMA - $\mathrm{SR}_{\mathrm{RSS}}$ control chart to identify a shift in the process location parameter. The Monte Carlo simulation

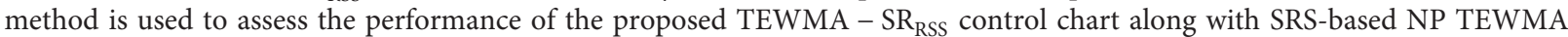
(TEWMA-SR), SRS-based NP TEWMA sign (TEWMA-SN), SRS-based TEWMA - $\bar{X}$, and RSS-based NP DEWMA-SR (DEWMA - $\mathrm{SR}_{\mathrm{RSS}}$ ) control charts. The study shows that the proposed TEWMA - $\mathrm{SR}_{\mathrm{RSS}}$ control chart is more efficient in identifying shifts (especially in small shifts) in the process location than the existing control charts. Finally, a real-life application is also provided for the practical implementation of the proposed TEWMA - SR $R_{R S S}$ control chart.

\section{Introduction}

Variations are an essential part of every manufacturing and service process, and these variations can be categorized as common and special cause variations. The common cause variations are harmless, and the mechanism or process that operates under these variations is called in-control (IC). However, the special cause variations affect the product quality, and the process that runs in these variations is referred to as out-of-control (OOC). Statistical process control (SPC) tool (e.g., cause-and-effect diagram, check sheet, control charts, histogram, Pareto chart, scatter diagram, and stratification) kit is famous for monitoring the shift (i.e., special cause variations) in the process parameters (location and/or dispersion). Compared to other SPC tools, the control charts got special attention because they are competent and easy to implement to detect the shift in the process parameters. Furthermore, the control charts can be classified into memoryless and memory-type depending on their design structures. Shewhart [1] introduced control charts known as the Shewhart control charts; these control charts are also named memoryless-type control charts. Shewhart control charts only use current information to detect a large shift quickly in the process parameters. Later 
on, Page [2] and Roberts [3] proposed cumulative sum (CUSUM) and exponentially weighted moving average (EWMA) control charts, respectively; these control charts are also known as memory-type. Memory-type control charts are famous for identifying a small-to-moderate shift in the process parameters.

Generally, for classical parametric control charts (i.e., Shewhart, CUSUM, and EWMA), the underlying process characteristics follow a normal distribution or any other specified probability distribution to identify a shift in the process parameters. Occasionally, there may be a situation when the underlying process characteristics do not follow any specific distribution or distribution of the underlying process characteristics is in doubt. In this case, nonparametric (NP) control charts are the robust substitute for parametric control charts. These control charts are convenient because their IC run length (RL) distribution is similar for all continuous distributions. The sign (SN) and Wilcoxon signed-rank (SR) are well-known NP techniques. The SN technique only required the assumption of continuity, whereas the SR method required symmetry assumption as well [4].

Bakir and Reynolds [5] introduced an NP CUSUM control chart (NPCUSUM-SR) based on the signed-rank statistic for the process location. Similarly, Amin and Searcy [6] developed an NP EWMA-SR control chart by combining the SR technique with the EWMA control chart to monitor the process location shift effectively. In the same lines, Bakir [7] presented the SR-based Shewhart control chart. After that, Balakrishnan et al. [8] suggested an NP Shewhart control chart using runs rules to make it statistically sensitive. Correspondingly, Yang and Cheng [9] and Yang et al. [10] introduced CUSUM-SN and EWMA-SN control charts, respectively, for effective process location monitoring. In the same way, Graham et al. [11] presented the design structure of the single observation-based NP EWMA control chart. Furthermore, Chakraborty et al. [12] developed the GWMA-SR control chart to improve the EWMA-SR control chart's ability to detect small shifts. Later on, Raza et al. [13] introduced the double NP EWMA-SR based (DEWMA-SR) control chart to monitor shift and showed that it is more sensitive than the EWMA-SR control chart. Likewise, Malela-Majika [14] developed new distribution-free control charts based on the Wilcoxon rank-sum test for efficiently monitoring the process location. Also, He et al. [15] designed an NP multivariate control chart for the time between events and amplitude data. This control chart is used to track the time intervals' location shifts and the amplitudes of an event.

In SPC, sampling techniques such as simple random sampling (SRS) and perfect ranked set sampling (RSS) are well known and are commonly used with SN and SR techniques to observe the underlying process data. Furthermore, both SRS and RSS sampling techniques are widely used with parametric and NP control charts. RSS is a necessary and valuable statistical technique commonly used in statistical quality control when the precise measurement of a selected unit is either difficult or prohibitively expensive and time-consuming [16]. It is self-evident that RSS efficiency is dependent on the precision with which the randomly selected units are defined. Errors in the ranking have a negative impact on the estimator's efficiency and result in imprecise estimates. Dell and Clutter [17] investigated the effect of error and imperfect ranking on mean estimator performance. They presented that even when imperfect rankings are used, the RSS mean estimator remains unbiased and outperforms the SRS mean estimator. However, the performance of the RSS remains superior to that of the imperfect RSS. Visual inspection of the study variable or based on the auxiliary variable may easily lead to ranking a small set of the selected units [18]. For example, hazardous waste sites with different contamination levels can be ranked by a visual inspection of soil discoloration, when the actual measurements of toxic chemicals and quantifying their environmental impact are very costly. In this regard, Salazar and Sinha [19] introduced RSS with control charts to monitor the process location shift. They demonstrated that the RSS-based control charts outperformed against SRSbased control charts. Similarly, Muttlak and Al-Sabah [20] and Abujiya and Muttlak [21] presented an RSS-based (median ranked set sampling and extreme ranked set sampling) and double RSS (DRSS) based control charts, respectively, to monitor the shift in the process location. Later, Al-Omari and Haq [22] proposed a Shewhart-type control chart based on the DRSS technique.

Haq et al. [23] suggested a maximum EWMA (MaxEWMA) control chart based on order DRSS (ODRSS) and order imperfect DRSS (OIDRSS) sampling techniques. In addition, they [24] also developed a new synthetic control chart for process location and dispersion using different RSS techniques. Furthermore, based on RSS, Haq and Khoo [25] developed synthetic EWMA and synthetic CUSUM control charts to monitor the process location. Recently, Abbas et al. [26] introduced an NP DEWMA-SR control chart using the RSS technique for process location monitoring, labelled as DEWMA - $\mathrm{SR}_{\mathrm{RSS}}$ control chart.

Similarly, in the SPC, modifications and enhancements are continually practiced to enhance the performance of the memory-type control charts. For example, Shamma and Shamma [27] extended the classical EWMA control chart and suggested a double EWMA (DEWMA) control chart for the process location. The DEWMA control chart is more responsive than the classical EWMA control chart. Anwar et al. [28] and Aslam and Anwar [29] introduced modifiedEWMA control charts, respectively, in the presence of auxiliary information and Bayesian methodology. Similarly, Anwar et al. [30] introduced auxiliary information-based (AIB) combined MEC for the simultaneous monitoring of process location and dispersion.

Recently, Chatterjee et al. [31] proposed a TEWMA control chart to monitor process dispersion shift. Later on, Alevizakos et al. [32] and Alevizakos et al. [4] suggested an NP TEWMA-SN (TEWMA-SN) and NP TEWMA-SR (TEWMA-SR) control charts, respectively. Furthermore, Alevizakos et al. [33] extended EWMA and DEWMA control charts and proposed a triple EWMA (TEWMA) control chart further to enhance the efficiency of the EWMA control chart. The TEWMA control chart is more responsive than the EWMA and DEWMA control charts to monitor small-to-moderate shifts in process location. 
As discussed earlier, the SRS-based TEWMA-SN and TEWMA-SR control charts are well known for timely monitoring of the quality characteristics easily available. Still, when selecting quality characteristics is either difficult or expensive and time-consuming, these SRS-based control charts fail to monitor the process efficiently. To monitor such quality characteristics, this study suggested an NP triple EWMA-SR control chart with the RSS technique (TEWMA - $\mathrm{SR}_{\mathrm{RSS}}$ ) for effectively monitoring the process location of a continuous and symmetric distribution. Various processing environments like normal, Contaminated normal $(\mathrm{CN})$, Laplace, Student's $t$, and Logistic are used to measure the RL characteristics of the proposed control chart. The average run length (ARL), the median of the run length (MDRL), and the standard deviation of the run length (SDRL) are assessed to determine the efficiency of the proposed control chart against other control charts. The sample points before the control chart signal are referred to as RL, and the RL's expected values are referred to as ARL. $A R L_{0}$ is the ARL of the IC process while $A R L_{1}$ is the ARL of the OOC process. A control chart is considered to be efficient when it has smaller $\mathrm{ARL}_{1}$ values as compared to its contestant at different shifts.

The remainder of the article is as follows: Section 2 presents the design structures of existing and the proposed control charts. Similarly, Section 3 offers the implementation of the proposed control chart. Also, the comparative study is given in Section 4, while Section 5 provides an illustrative example related to the proposed control chart. Finally, Section 6 offers the summary, conclusions, and recommendations of the study.

\section{Existing and Proposed Methods}

This section explains the design structures of some existing and the proposed control charts. For example, Section 2.1 presents the RSS-based Wilcoxon signed-rank statistic. Similarly, methodologies of the NP EWMA SR with RSS (EWMA - SR RSS $)$ and DEWMA - $\mathrm{SR}_{\mathrm{RSS}}$ control charts are given in Sections 2.2 and 2.3, respectively. Finally, the design structure of the proposed TEWMA - $\mathrm{SR}_{\mathrm{RSS}}$ control chart is provided in Section 2.4.

2.1. RSS-Based Wilcoxon Signed-Rank Statistic. Mclntyre [16] gives the idea of the RSS technique for data collection. Suppose RSS of size $n$ is taken from a quality variable $X_{q j(h)}$, where $q=1,2,3, \ldots, j=1,2,3, \ldots, n$, and $h=1,2,3, \ldots$, $m . q$ denotes each sample, $j$ denotes the number of observations, and $h$ denotes the number of cycles used in the RSS technique. $r=n m$ is the number of iterations used in the RSS technique with a sample of size $n$ and $m$ number of cycles. Let $R_{q j(h)}^{+}=\left|X_{q j(h)}-\varphi_{0}\right|$, where $R_{q j(h)}^{+}$denote the ranks of absolute deviations of RSS values $\left(X_{q j(h)}\right)$ from median $\left(\varphi_{0}\right)$. According to Kim and Kim [34], the statistic of RSS under SR can be written as

$$
\mathrm{SR}_{\mathrm{RSS}_{q}}=\sum_{j=1}^{n} \sum_{h=1}^{m} \operatorname{sign}\left(X_{q j(h)}-\varphi_{0}\right) R_{q j(h)}^{+},
$$

where

$$
\operatorname{sign}\left(X_{q j(h)}-\varphi_{0}\right)= \begin{cases}1, & \text { if } X_{q j(h)}-\varphi_{0}>0, \\ 0, & \text { if } X_{q j(h)}-\varphi_{0}=0 \\ -1, & \text { if } X_{q j(h)}-\varphi_{0}<0 .\end{cases}
$$

The mean and variance of the statistic $\mathrm{SR}_{\mathrm{RSS}}$ are $E\left(\mathrm{SR}_{\mathrm{RSS}}\right)=0$ and $\operatorname{var}\left(\mathrm{SR}_{\mathrm{RSS}}\right)=((r(r+1)(2 r+1)) / 6) \omega_{0}^{2}$, respectively [34]. The $\omega_{0}^{2}$ can be defined as follows:

$$
\omega_{0}^{2}=1-\frac{4}{n} \sum_{j=1}^{n}\left(F_{k}(0)-\frac{1}{2}\right)^{2}
$$

where the values of $F_{k}(0)$ can be obtained by solving the following expression for $j=1,2, \ldots, n$ :

$$
F_{k}(0)=\frac{r !}{(j-1) !(r-j) !} \int_{-\propto}^{0} F(t)^{j-1}(1-F(t))^{r-j} f(t) \mathrm{d} t,
$$

whereas $\omega_{0}^{2}$ is used to enhance the performance of the control chart. The values of $\omega_{0}^{2}$ at different sample sizes taken from [35] are given as $\left(n, \omega_{0}^{2}\right)=(2,0.750),(3,0.625),(4$, $0.547),(5,0.490),(6,0.451),(7,0.416),(8,0.393),(9,0.371)$, and (10, 0.352).

2.2. EWMA - SR RSS Control Chart. The EWMA - $\mathrm{SR}_{\mathrm{RSS}}$ control chart is more sensitive for detecting shifts in the process location/median than the NPEWMA-SR control chart. The plotting statistic of the EWMA - $\mathrm{SR}_{\mathrm{RSS}}$ control chart based on equation (1) is defined as

$$
E_{q}=\lambda \mathrm{SR}_{\mathrm{RSS}_{q}}+(1-\lambda) E_{q-1},
$$

where $\lambda \in(0,1]$ is a smoothing constant and the initial value of the $E_{q}$ is equal to 0 . The mean and variance of the statistic $E_{q}$ are $E\left(E_{q}\right)=0$ and $\operatorname{var}\left(E_{q}\right)=(\lambda /(2-\lambda))\left(1-(1-\lambda)^{2 q}\right)$ $(r(r+1)(2 r+1) / 6) \omega_{0}^{2}$, respectively. The variance of $E_{q}$ is reduced to $(\lambda /(2-\lambda))(r(r+1)(2 r+1) / 6) \omega_{0}^{2}$, when the term $\left(1-(1-\lambda)^{2 q}\right)$ approaches to unity. The control limits of the EWMA - $\mathrm{SR}_{\mathrm{RSS}}$ control chart (see Table 1) are defined below:

$$
\left.\begin{array}{l}
\mathrm{UCL}_{\mathrm{EWMA}_{\mathrm{SR}} \mathrm{RSS}}=+L \sqrt{\frac{\lambda}{2-\lambda}\left(\frac{r(r+1)(2 r+1)}{6}\right) \omega_{0}^{2}} \\
\mathrm{LCL}_{\mathrm{EWMA}^{2} \mathrm{SR}_{\mathrm{RSS}}}=-L \sqrt{\frac{\lambda}{2-\lambda}\left(\frac{r(r+1)(2 r+1)}{6}\right) \omega_{0}^{2}}
\end{array}\right\},
$$

where $L$ is the width of the control limits, which helps to determine $\mathrm{ARL}_{0}$. The process will be $\mathrm{OOC}$ if any $E_{q}>\mathrm{UCL}_{\mathrm{EWMA} \mathrm{SR}_{\mathrm{RSS}}}$ or $E_{q}<\mathrm{LCL}_{\mathrm{EWMA} \mathrm{SR}_{\mathrm{RSS}}}$; otherwise, it will remain in an IC state.

2.3. DEWMA - SR RSS Control Chart. Abbas et al. [26] introduced the following plotting statistics of the DEWMA - SR $R_{R S S}$ control chart: 


$$
\left.\begin{array}{l}
E_{q}=\lambda \mathrm{SR}_{\mathrm{RSS}_{q}}+(1-\lambda) E_{q-1} \\
\mathrm{DE}_{q}=\lambda E_{q}+(1-\lambda) \mathrm{DE}_{q-1}
\end{array}\right\},
$$

The mean and variance of $\mathrm{DE}_{q}\left(\mathrm{DEWMA}-\mathrm{SR}_{\mathrm{RSS}}\right.$ control chart) for IC process are $E\left(\mathrm{DE}_{q}\right)=0$ and $\operatorname{var}\left(\mathrm{DE}_{q}\right)$ $=\left((r(r+1)(2 r+1) / 6) \omega_{0}^{2}\right) \lambda^{4}\left(\left(1+\lambda^{2}-\left(q^{2}+2 q+1\right) \lambda^{2 q}+(2\right.\right.$ $\left.\left.\left.q^{2}+2 q-1\right) \lambda^{2 q+2}-q^{2}\right) /\left(1-\lambda^{2}\right)^{3}\right)$, respectively. The control limits of the DEWMA - SR $\mathrm{RSS}$ control chart can be designed as

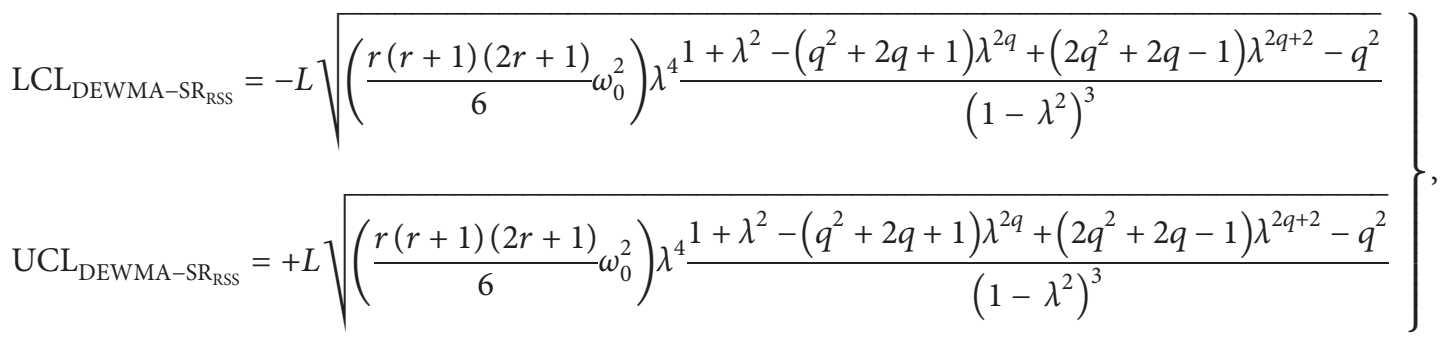

where $L$ is the control chart width and the initial value of the $\mathrm{DE}_{q}=0$. The process will be OOC when $\mathrm{LCL}_{\mathrm{DEWMA}-\mathrm{SR}_{\mathrm{RSS}}}>$ $\mathrm{DE}_{q}$ or $\mathrm{UCL}_{\mathrm{DEWMA}-\mathrm{SR}_{\mathrm{RSS}}}<\mathrm{DE}_{q}$; otherwise, it will remain in an IC state.

2.4. Proposed TEWMA - SR $R_{R S S}$ Control Chart. This section offers a proposed control chart structure. In more detail, the proposed TEWMA - $\mathrm{SR}_{\mathrm{RSS}}$ control chart is designed for monitoring the process location shift. The plotting statistics of the proposed TEWMA - $\mathrm{SR}_{\mathrm{RSS}}$ control chart are defined as

$$
\left.\begin{array}{l}
E_{q}=\lambda \mathrm{SR}_{\mathrm{RSS}_{q}}+(1-\lambda) E_{q-1} \\
\mathrm{DE}_{q}=\lambda E_{q}+(1-\lambda) \mathrm{DE}_{q-1} \\
\mathrm{TE}_{q}=\lambda \mathrm{DE}_{q}+(1-\lambda) \mathrm{TE}_{q-1}
\end{array}\right\} \text {, }
$$

The initial values of $E_{0}, \mathrm{DE}_{0}$, and $\mathrm{TE}_{0}$ are all equal to 0 (zero). The mean and variance of the statistic $\mathrm{TE}_{q}$ under IC state are $E\left(\mathrm{TE}_{q}\right)=0$ and

$$
\begin{aligned}
\operatorname{var}\left(\mathrm{TE}_{q}\right)= & {\left[\frac{\theta^{3} \lambda^{6}}{4}\left[-\frac{q\left(q^{2}-1\right)(q-2)^{\theta^{q}-3}}{1-\theta}-\frac{4 q\left(q^{2}-1\right) \theta^{q-2}}{(1-\theta)^{2}}-\frac{12 q(q+1) \theta^{q-1}}{(1-\theta)^{3}}-\frac{24(q+1) \theta^{q}}{(1-\theta)^{4}}+\frac{24\left(1-\theta^{q+1}\right)}{(1-\theta)^{5}}\right]\right.} \\
& +2 \theta^{2} \lambda^{6}\left[-\frac{q\left(q^{2}-1\right) \theta^{q-2}}{1-\theta}-\frac{3 q(q+1) \theta^{q-1}}{(1-\theta)^{2}}-\frac{6(q+1) \theta^{q}}{(1-\theta)^{3}}+\frac{6\left(1-\theta^{q+1}\right)}{(1-\theta)^{4}}\right] \\
& +\frac{7 \theta \lambda^{6}}{2}\left[-\frac{q(q+1) \theta^{q-1}}{1-\theta}-\frac{2(q+1) \theta^{q}}{(1-\theta)^{2}}+\frac{2\left(1-\theta^{q+1}\right)}{(1-\theta)^{3}}\right]+\lambda^{6}\left[\frac{1-\theta^{q+1}}{(1-\theta)^{2}}-\frac{(q+1) \theta^{q}}{(1-\theta)}\right] \frac{n(n+1)(2 n+1)}{6} \omega_{0}^{2},
\end{aligned}
$$

respectively, where $\theta=(1-\lambda)^{2}$. The time-varying control

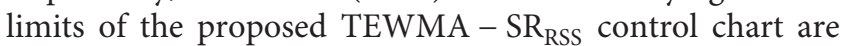
specified as

$$
\left.\begin{array}{l}
\mathrm{LCL}_{\left(\mathrm{TEWMA}_{\mathrm{SRS}}\right) q}=-L \sqrt{\operatorname{var}\left(\mathrm{TE}_{q}\right)} \\
\mathrm{UCL}_{\left(\mathrm{TEWMA}_{\mathrm{SR}} \mathrm{RSS}_{\mathrm{S}}\right)}=+L \sqrt{\operatorname{var}\left(\mathrm{TE}_{q}\right)}
\end{array}\right\},
$$

where $L$ is the width of the control limits. For large values of $q$, we have $\operatorname{var}\left(\mathrm{TE}_{q}\right)=\left[\left(6(1-\lambda)^{6} \lambda /(2-\lambda)^{5}\right)+\left(12(1-\lambda)^{4}\right.\right.$ $\left.\left.\lambda^{2} /(2-\lambda)^{4}\right)+\left(7(1-\lambda)^{2} \lambda^{3} /(2-\lambda)^{3}\right)+\left(\lambda^{4} /(2-\lambda)^{2}\right)\right](n(n$ $+1)(2 n+1) / 6) \omega_{0}^{2}$ and the asymptotic control limits become

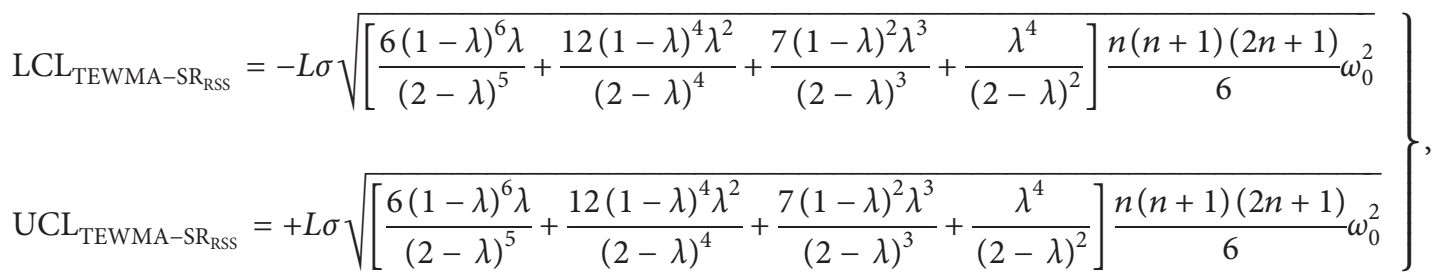


The process goes OOC if any $\mathrm{TE}_{q}>\mathrm{UCL}_{\mathrm{TEWMA} \mathrm{SR}_{\mathrm{RSS}}}$ or

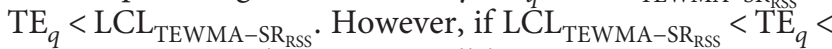
$\mathrm{UCL}_{\mathrm{TEWMA}-\mathrm{SR}_{\mathrm{RSS}}}$, the process will be in IC state.

\section{Implementation of the Proposed TEWMA - SR RSS $_{\text {Control Chart }}$}

This section provides the implementation of the proposed TEWMA - $\mathrm{SR}_{\mathrm{RSS}}$ control chart. Section 3.1 describes the choice of design parameters. Similarly, Section 3.2 presents the evaluation of the proposed control chart.

3.1. Choice of Design Parameters. $\lambda$ and $L$ are the design parameters of the proposed TEWMA - $\mathrm{SR}_{\mathrm{RSS}}$ control chart. The control chart width $L$ is chosen with various values of $\lambda$, such that the prespecified $\mathrm{ARL}_{0}$ is obtained. The best subgroup size is between 5 and 10 , depending on $\mathrm{ARL}_{0}$ and shift size [5]. Different RL properties are assessed to study the performance behavior of the proposed TEWMA $-\mathrm{SR}_{\mathrm{RSS}}$ control chart with $\lambda \in(0.05,0.10,0.25,0.50)$ and $n \in(5,10)$. $\mathrm{ARL}_{0}$ is assumed to be 370 for this study.

3.2. Evaluation. This section provides the RL characteristics of the different distributions (normal and non-normal distributions) for IC robustness and IC performance of the proposed control chart. The proposed TEWMA - $S R_{R S S}$ control chart's RL features are evaluated using normal and non-normal distributions.

3.2.1. Evaluation of the Run-Length Distribution. The performance of the proposed control chart can be assessed in both normal and non-normal symmetric distributions. The distributions used in this analysis are as follows:

(i) Standard normal distribution, i.e., $N(0,1)$

(ii) Double exponential (Laplace) distribution, i.e., $\operatorname{DE}(0,(1 / \sqrt{2}))$

(iii) Heavy tail Student's $t$ distribution $t_{v}$, with the degree of freedom $(v)=4$ and 8

(iv) The logistic distribution, i.e., $\operatorname{LOG}(0,(\sqrt{3} / \pi))$

(v) Contaminated normal $(\mathrm{CN})$ distribution, which is a mixture of $N\left(0, \sigma_{1}^{2}\right)$ and $N\left(0, \sigma_{0}^{2}\right)$, i.e., $(1-\alpha)$ $N\left(0, \sigma_{1}^{2}\right)+\alpha N\left(0, \sigma_{0}^{2}\right)$, with $\alpha$ such that $(1-\alpha) \sigma_{1}^{2}$ $+\alpha \sigma_{0}^{2}=1$, where $\alpha=0.05$ and $\left(\sigma_{1} / \sigma_{0}\right)=2$. For comparison purpose, all the considered distributions in this analysis were reparametrized with mean zero and unit standard deviation. The probability density functions of the distributions used in this study are shown in Table 2.

3.2.2. Monte Carlo Simulation. The Monte Carlo simulation is used to obtain the RL characteristics of the proposed control chart. The simulation algorithm is developed in $R$ software to compute the RL characteristics. $10^{4}$ random samples of the size $n>1$ from any distribution covered by the study for a shift $(\delta)$ were generated. The simulation algorithm to determine the nominal values of $\mathrm{ARL}_{0}$ under various distributions can be explained in the following steps [36]:

(a) An finite loop is practiced to generate samples from different distributions

(b) Select a particular value for $\lambda$

(c) Determine the $\mathrm{TE}_{q}$ plotting statistic offered in equation (9)

(d) Let us assume $L=3$

(e) Calculate the UCL $\mathrm{UEWMA}_{\mathrm{TER}}$ and $\mathrm{LCL}_{\mathrm{TEWMA}} \mathrm{SR}_{\mathrm{RSS}}$ from equation (11)

(f) Plot the $\mathrm{TE}_{q}$ statistic against control limits in step (e) over $q$

(g) If the $\mathrm{TE}_{q}>\mathrm{UCL}_{\mathrm{TEWMA} \mathrm{SR}_{\mathrm{RSS}}}$ or $\mathrm{TE}_{q}<$ $\mathrm{LCL}_{\text {TEWMA-SR }}$,SS , record the sample number of $\mathrm{TE}_{q}$ statistic as an RL. For example, at $q=255$, if $\mathrm{TE}_{q}>\mathrm{UCL}_{\mathrm{TEWMA} \mathrm{SR}_{\mathrm{RSS}}}$ or $\mathrm{TE}_{q}<\mathrm{LCL}_{\mathrm{TEWMA}-\mathrm{SR}_{\mathrm{RSS}}}$ record 255 as a first RL.

(h) Repeat steps from (a) to (g) for $10^{5}$ times and record RLs.

(i) Compute the average of $10^{5}$ times noted RLs, which is called $\mathrm{ARL}_{0}$.

(j) If $\mathrm{ARL}_{0}=400$; otherwise, adjust $L$ (i.e., $L<3$ or $L>3$ ) constant accordingly in step (d) and repeat steps from (a) to (i) to obtain $\mathrm{ARL}_{0}=400$.

(k) To compute the $\mathrm{ARL}_{1}$ values, again generate samples from relevant distribution and repeat steps from (b) to (i).

3.2.3. In-Control Robustness of the Proposed Control Chart. The EWMA and DEWMA $-\bar{X}$ control charts have the limitation that their IC RL features do not remain the same for all continuous distributions. The IC RL characteristics of the NP control chart's characteristics remain the same for all symmetric continuous distributions [4]. The simulated results of the proposed control chart with different combinations of $\lambda$ and $L$ to obtain the nominal $\mathrm{ARL}_{0}=370$ are shown in Tables 3 and 4. The IC RL distribution of the proposed TEWMA-SR $R_{R S S}$ control chart seems to be similar for all distributions used in this study. The RL distribution is positively skewed when ARL $>$ MDRL.

3.2.4. Out-of-Control Performance. OOC performance of the proposed control chart can be discussed under perfect RSS and imperfect RSS. The OOC control chart's efficiency is primarily compared, and it demonstrates how sensitive the control chart is to detect shifts in process parameters. Tables 3 and 4 show the OOC performance of the proposed TEWMA - $\mathrm{SR}_{\mathrm{RSS}}$ control chart for the various distributions evaluated in this study. For small and moderate shifts, the OOC performance of the proposed TEWMA - $\mathrm{SR}_{\mathrm{RSS}}$ control chart increases as $n$ increases; i.e., $\mathrm{ARL}_{1}$ values decrease by increasing $n$. The proposed scheme's RL values reduce with the rise in $n$ and $m$ values. The OOC RL performance of the 


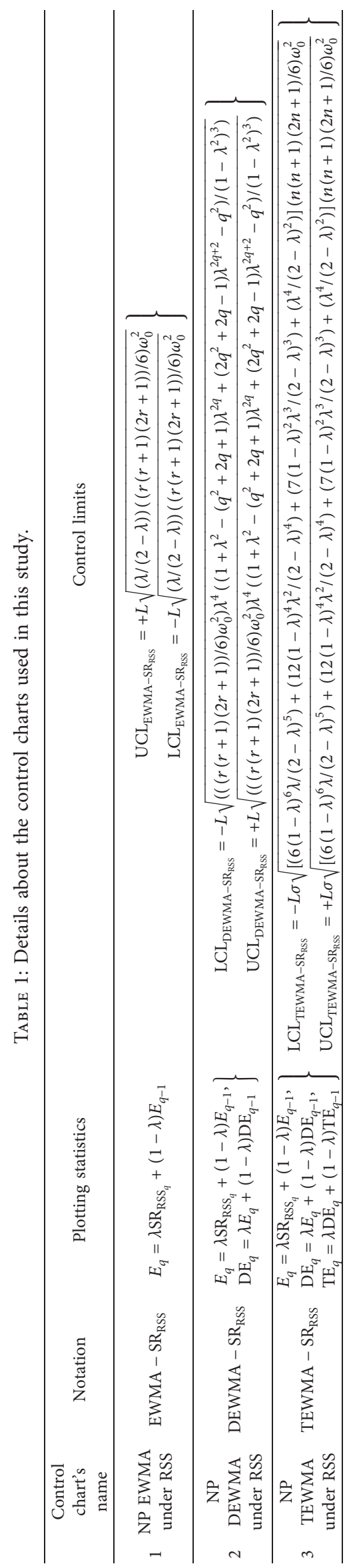


TABLE 2: PDFs of the continuous distributions used for this study.

(i) Standard normal $f(X)=\left(e^{\left(-x^{2} / 2\right)} / \sqrt{2 \pi}\right)$, where $X \in \Re, M_{0}=0$, and $\sigma^{2}=1$

(ii) Student's $t_{v} f(X)=(\Gamma((v+1) / 2) /(\Gamma(v / 2) \sqrt{v \pi}))\left(1+\left(X^{2} / v\right)\right)^{((v+1) / 2)}$, where $X \in \mathfrak{R}, M_{0}=0$, and $\sigma^{2}=(v /(v-2))$ and $v=4,8$ are

taken

(iii) Logistic $f(X)=e^{(-\pi X) / \sqrt{3}} /\left((\sqrt{3} / \pi)\left(1+e^{(-\pi X) / \sqrt{3}}\right)^{2}\right)$, where $X \in \Re, M_{0}=0$, and $\sigma^{2}=\left(3 / \pi^{2}\right)$

(iv) Laplace $f(X)=(1 / 2) e^{-|X|}$, where $X \in \mathfrak{R}, M_{0}=0$, and $\sigma^{2}=(1 / 2)$

(v) Contaminated normal $(\mathrm{CN}) f(X)=\left(0.95 e^{\left(-X^{2} / 2\right)} / \sqrt{2 \pi}\right)+\left(0.05 e^{-\left(X^{2} / 2 \sigma_{0}^{2}\right)} / \sigma_{0 \sqrt{2 \pi}}\right)$, where $X \in \Re, M_{0}=0$, and $\sigma^{2}=0.95+0.05 \sigma_{0}^{2}$

TABLE 3: RL characteristics of the proposed TEWMA $-\mathrm{SR}_{\mathrm{RSS}}$ control chart under different distributions with nominal $\mathrm{ARL}_{0}=370$ and $n=5$.

\begin{tabular}{|c|c|c|c|c|c|c|c|c|c|c|c|c|c|c|c|c|}
\hline \multirow{2}{*}{$(\lambda, L)$} & & & \multicolumn{14}{|c|}{0} \\
\hline & & & 0.00 & 0.025 & 0.03 & 0.05 & 0.075 & 0.10 & 0.15 & 0.20 & 0.25 & 0.50 & 0.75 & 1.00 & 1.50 & 2.00 \\
\hline \multirow{18}{*}{$(0.05,1.585)$} & \multirow{3}{*}{$N(0,1)$} & ARL & 371.11 & 206.77 & 168.62 & 92.67 & 50.53 & 33.91 & 19.12 & 12.02 & 7.94 & 2.35 & 1.37 & 1.08 & 1.01 & 1.00 \\
\hline & & MDRL & 218.00 & 131.00 & 111.00 & 70.00 & 41.00 & 28.00 & 17.00 & 9.00 & 7.00 & 2.00 & 1.00 & 1.00 & 1.00 & 1.00 \\
\hline & & SDRL & 453.10 & 234.01 & 183.70 & 90.33 & 49.06 & 29.15 & 15.72 & 10.54 & 6.62 & 1.77 & 0.75 & 0.31 & 0.08 & 0.00 \\
\hline & \multirow{3}{*}{$\mathrm{CN}$} & ARL & 369.92 & 212.31 & 164.04 & 94.31 & 51.93 & 35.63 & 19.37 & 12.06 & 8.35 & 2.51 & 1.46 & 1.14 & 1.01 & 1.00 \\
\hline & & MDRL & 217.50 & 139.00 & 113.00 & 65.00 & 40.00 & 27.50 & 16.00 & 9.00 & 6.00 & 2.00 & 1.00 & 1.00 & 1.00 & 1.00 \\
\hline & & SDRL & 441.07 & 235.19 & 179.28 & 97.35 & 50.15 & 33.35 & 16.16 & 10.77 & 7.50 & 2.08 & 0.87 & 0.43 & 0.13 & 0.04 \\
\hline & \multirow{3}{*}{ Logistic } & ARL & 371.69 & 189.29 & 156.51 & 80.72 & 49.37 & 30.49 & 16.21 & 10.27 & 7.20 & 2.18 & 1.31 & 1.10 & 1.01 & 1.00 \\
\hline & & MDRL & 230.50 & 118.00 & 112.00 & 62.00 & 41.00 & 25.00 & 13.00 & 8.00 & 6.00 & 1.00 & 1.00 & 1.00 & 1.00 & 1.00 \\
\hline & & SDRL & 429.72 & 216.77 & 159.50 & 83.63 & 44.70 & 26.42 & 14.26 & 8.94 & 6.39 & 1.61 & 0.66 & 0.33 & 0.11 & 0.03 \\
\hline & \multirow{4}{*}{ Laplace } & ARL & 371.65 & 158.45 & 123.59 & 63.49 & 34.18 & 22.54 & 12.25 & 7.52 & 5.48 & 2.01 & 1.32 & 1.12 & 1.03 & 1.00 \\
\hline & & MDRL & 237.00 & 106.50 & 87.00 & 50.00 & 28.00 & 18.00 & 10.00 & 5.00 & 4.00 & 1.00 & 1.00 & 1.00 & 1.00 & 1.00 \\
\hline & & SDRL & 428.92 & 173.36 & 126.83 & 60.33 & 30.92 & 20.29 & 10.72 & 6.89 & 4.85 & 1.50 & 0.66 & 0.38 & 0.17 & 0.06 \\
\hline & & ARL & 369.29 & 215.22 & 187.04 & 111.34 & 62.00 & 41.65 & 23.55 & 15.39 & 10.43 & 3.34 & 1.79 & 1.36 & 1.09 & 1.02 \\
\hline & \multirow[t]{3}{*}{$t_{4}$} & MDRL & 232.50 & 126.50 & 123.00 & 83.00 & 47.00 & 33.00 & 20.00 & 12.00 & 8.00 & 2.00 & 1.00 & 1.00 & 1.00 & 1.00 \\
\hline & & SDRL & 423.91 & 254.75 & 210.06 & 111.40 & 59.02 & 39.91 & 20.45 & 12.90 & 9.58 & 2.79 & 1.31 & 0.70 & 0.34 & 0.17 \\
\hline & & ARL & 370.51 & 202.75 & 174.37 & 99.36 & 58.92 & 37.64 & 20.00 & 13.33 & 8.89 & 2.80 & 1.58 & 1.20 & 1.03 & 1.01 \\
\hline & \multirow[t]{2}{*}{$t_{8}$} & MDRL & 231.00 & 128.00 & 118.00 & 70.00 & 45.00 & 33.00 & 15.00 & 10.00 & 7.00 & 2.00 & 1.00 & 1.00 & 1.00 & 1.00 \\
\hline & & SDRL & 455.11 & 247.02 & 189.65 & 102.22 & 55.97 & 32.31 & 17.50 & 11.80 & 7.86 & 2.40 & 1.03 & 0.54 & 0.17 & 0.12 \\
\hline \multirow{18}{*}{$(0.10,1.837)$} & \multirow{3}{*}{$N(0,1)$} & & 58 & 39.49 & 200 & 4 & 57.60 & 38.66 & 21.58 & 13.26 & 9.19 & 3.09 & 1.75 & 1.28 & .03 & 1.00 \\
\hline & & MDRL & 241.50 & 164.00 & 143.00 & 75.50 & 44.00 & 32.00 & 19.00 & 12.00 & 8.00 & 2.00 & 1.00 & 1.00 & 1.00 & 1.00 \\
\hline & & SDRL & 399.98 & 252.12 & 200.34 & 104.09 & 52.29 & 31.47 & 16.68 & 9.58 & 6.52 & 2.10 & 0.97 & 0.54 & 0.18 & 0.03 \\
\hline & \multirow{3}{*}{$\mathrm{CN}$} & ARL & 371.69 & 222.24 & 189.81 & 110.71 & 64.19 & 40.58 & 21.02 & 13.65 & 9.67 & 3.28 & 1.79 & 1.32 & 1.05 & 1.01 \\
\hline & & MDRL & 240.50 & 155.00 & 136.50 & 75.00 & 48.00 & 34.00 & 18.00 & 12.00 & 8.00 & 3.00 & 2.00 & 1.00 & 1.00 & 1.00 \\
\hline & & SDRL & 400.58 & 233.16 & 189.80 & 111.14 & 60.27 & 32.09 & 16.17 & 9.93 & 6.98 & 2.33 & 1.02 & 0.60 & 0.22 & 0.08 \\
\hline & \multirow{4}{*}{ Logistic } & ARL & 369.89 & 220.35 & 179.15 & 97.85 & 52.17 & 35.77 & 18.26 & 11.25 & 8.24 & 2.73 & 1.67 & 1.25 & 1.04 & 1.01 \\
\hline & & MDRL & 257.50 & 150.00 & 124.00 & 72.00 & 40.00 & 29.00 & 16.00 & 10.00 & 7.00 & 2.00 & 1.00 & 1.00 & 1.00 & 1.00 \\
\hline & & SDRL & 379.94 & 229.75 & 177.94 & 90.99 & 47.57 & 29.96 & 13.90 & 8.42 & 5.94 & 1.80 & 0.89 & 0.52 & 0.20 & 0.07 \\
\hline & & ARL & 369.00 & 179.37 & 145.59 & 72.15 & 39.00 & 25.62 & 14.36 & 8.83 & 6.40 & 2.42 & 1.52 & 1.27 & 1.05 & 1.01 \\
\hline & \multirow[t]{3}{*}{ Laplace } & MDRL & 257.50 & 117.50 & 103.00 & 54.00 & 31.00 & 22.00 & 13.00 & 8.00 & 5.00 & 2.00 & 1.00 & 1.00 & 1.00 & 1.00 \\
\hline & & SDRL & 379.94 & 191.51 & 141.81 & & 33.78 & 19.39 & 10.66 & 6.28 & 4.77 & 1.58 & 0.83 & 0.56 & 0.23 & 0.10 \\
\hline & & ARL & 369.26 & 257.35 & 229.58 & 142.19 & 81.25 & 48.90 & 26.11 & 16.68 & 12.29 & 3.98 & 2.27 & 1.60 & 1.16 & 1.04 \\
\hline & \multirow[t]{3}{*}{$t_{4}$} & MDRL & 239.00 & 166.50 & 154.00 & 102.00 & 59.50 & 38.00 & 23.00 & 14.00 & 11.00 & 3.00 & 2.00 & 1.00 & 1.00 & 1.00 \\
\hline & & SDRL & 382.67 & 289.42 & 240.47 & 134.47 & 75.25 & 42.73 & 19.56 & 12.46 & 8.78 & 2.78 & 1.43 & 0.90 & 0.43 & 0.22 \\
\hline & & ARL & 370.13 & 241.63 & 203.62 & 125.90 & 67.99 & 44.76 & 22.63 & 15.92 & 10.57 & 3.42 & 1.92 & 1.38 & 1.06 & 1.01 \\
\hline & \multirow[t]{2}{*}{$t_{8}$} & MDRL & 250.00 & 169.50 & 139.00 & 89.50 & 52.00 & 37.00 & 20.00 & 14.00 & 9.00 & 3.00 & 2.00 & 1.00 & 1.00 & 1.00 \\
\hline & & SDRL & 417.20 & 260.53 & 202.72 & 129.48 & 59.38 & 36.44 & 17.13 & 11.11 & 7.52 & 2.42 & 1.14 & 0.62 & 0.27 & 0.08 \\
\hline
\end{tabular}


TABle 3: Continued.

\begin{tabular}{|c|c|c|c|c|c|c|c|c|c|c|c|c|c|c|c|c|}
\hline \multirow{2}{*}{$(\lambda, L)$} & & & \multicolumn{14}{|c|}{$\delta$} \\
\hline & & & 0.00 & 0.025 & 0.03 & 0.05 & 0.075 & 0.10 & 0.15 & 0.20 & 0.25 & 0.50 & 0.75 & 1.00 & 1.50 & 2.00 \\
\hline \multirow{18}{*}{$(0.25,2.204)$} & \multirow{3}{*}{$N(0,1)$} & ARL & 370.74 & 284.31 & 253.51 & 151.84 & 88.78 & 54.57 & 24.92 & 15.57 & 10.38 & 3.59 & 2.09 & 1.44 & 1.07 & 1.01 \\
\hline & & MDRL & 267.50 & 199.50 & 167.00 & 108.50 & 64.00 & 38.50 & 19.00 & 13.00 & 9.00 & 3.00 & 2.00 & 1.00 & 1.00 & 1.00 \\
\hline & & SDRL & 369.16 & 291.85 & 267.11 & 151.35 & 88.66 & 50.80 & 20.17 & 11.01 & 6.99 & 2.13 & 1.06 & 0.63 & 0.26 & 0.08 \\
\hline & \multirow{3}{*}{$\mathrm{CN}$} & ARL & 371.10 & 293.39 & 250.56 & 154.48 & 88.79 & 57.94 & 27.45 & 15.74 & 11.22 & 3.78 & 2.12 & 1.58 & 1.13 & 1.02 \\
\hline & & MDRL & 273.00 & 214.50 & 172.50 & 109.50 & 64.00 & 42.00 & 21.00 & 13.00 & 10.00 & 3.50 & 2.00 & 1.00 & 1.00 & 1.00 \\
\hline & & SDRL & 366.10 & 279.85 & 252.76 & 141.01 & 83.90 & 53.54 & 23.59 & 11.44 & 7.55 & 2.10 & 1.08 & 0.75 & 0.37 & 0.15 \\
\hline & \multirow{3}{*}{ Logistic } & ARL & 370.38 & 271.57 & 242.25 & 137.58 & 77.87 & 47.20 & 22.34 & 13.59 & 9.28 & 3.23 & 1.95 & 1.44 & 1.08 & 1.02 \\
\hline & & MDRL & 265.50 & 180.50 & 169.50 & 103.00 & 58.00 & 36.00 & 18.50 & 12.00 & 8.00 & 3.00 & 2.00 & 1.00 & 1.00 & 1.00 \\
\hline & & SDRL & 360.62 & 282.24 & 242.01 & 135.38 & 69.56 & 40.44 & 16.29 & 9.51 & 5.85 & 1.87 & 1.02 & 0.65 & 0.28 & 0.13 \\
\hline & & ARL & 370.38 & 230.11 & 199.04 & 105.76 & 52.44 & 30.72 & 15.08 & 10.52 & 7.07 & 2.82 & 1.83 & 1.35 & 1.10 & 1.02 \\
\hline & \multirow[t]{2}{*}{ Laplace } & MDRL & 265.50 & 156.50 & 144.00 & 75.00 & 38.50 & 23.00 & 13.00 & 9.00 & 6.00 & 3.00 & 2.00 & 1.00 & 1.00 & 1.00 \\
\hline & & SDRL & 360.62 & 223.94 & 196.34 & 100.81 & 47.67 & 26.97 & 11.19 & 7.14 & 4.41 & 1.68 & 0.97 & 0.65 & 0.33 & 0.17 \\
\hline & \multirow{4}{*}{$t_{4}$} & ARL & 371.04 & 304.19 & 282.63 & 193.67 & 113.38 & 72.96 & 32.78 & 19.37 & 13.04 & 4.52 & 2.52 & 1.85 & 1.28 & 1.11 \\
\hline & & MDRL & 251.00 & 208.50 & 200.00 & 136.50 & 80.00 & 51.00 & 25.00 & 15.00 & 11.00 & 4.00 & 2.00 & 2.00 & 1.00 & 1.00 \\
\hline & & SDRL & 367.97 & 313.54 & 280.19 & 186.75 & 112.53 & 70.92 & 27.16 & 16.71 & 8.95 & 2.58 & 1.48 & 0.98 & 0.55 & 0.36 \\
\hline & & ARL & 370.07 & 116.22 & 108.76 & 81.73 & 54.05 & 36.08 & 19.70 & 12.12 & 8.85 & 3.08 & 1.89 & 1.35 & 1.07 & 1.01 \\
\hline & \multirow[t]{2}{*}{$t_{8}$} & MDRL & 261.50 & 82.50 & 74.00 & 60.50 & 39.00 & 26.00 & 16.00 & 11.00 & 8.00 & 3.00 & 2.00 & 1.00 & 1.00 & 1.00 \\
\hline & & SDRL & 366.37 & 115.99 & 114.08 & 80.49 & 53.32 & 36.90 & 16.95 & 9.09 & 6.20 & 1.92 & 1.04 & 0.59 & 0.26 & 0.11 \\
\hline \multirow{18}{*}{$(0.50,2.491)$} & \multirow{3}{*}{$N(0,1)$} & ARL & 08 & 1.27 & 97 & 0.28 & 137.41 & 02 & 38.24 & 22.17 & 12.96 & 3.72 & 2.12 & 1.47 & 1.07 & 1.00 \\
\hline & & MDRL & 252.50 & 207.50 & 204.50 & 147.50 & 97.00 & 63.00 & 28.00 & 16.00 & 10.00 & 3.00 & 2.00 & 1.00 & 1.00 & 1.00 \\
\hline & & SDRL & 381.21 & 322.22 & 323.87 & 205.59 & 134.81 & 82.86 & 35.61 & 19.95 & 10.58 & 2.13 & 1.09 & 0.67 & 0.26 & 0.04 \\
\hline & \multirow{3}{*}{$\mathrm{CN}$} & ARL & 369.07 & 317.34 & 298.10 & 218.94 & 142.93 & 91.04 & 41.29 & 22.56 & 14.44 & 3.95 & 2.29 & 1.54 & 1.12 & 1.02 \\
\hline & & MDRL & 254.00 & 223.00 & 210.00 & 150.00 & 99.00 & 65.00 & 30.00 & 17.00 & 11.00 & 4.00 & 2.00 & 1.00 & 1.00 & 1.00 \\
\hline & & SDRL & 371.98 & 317.49 & 304.17 & 207.28 & 141.42 & 86.41 & 38.00 & 20.71 & 11.92 & 2.38 & 1.17 & 0.74 & 0.35 & 0.13 \\
\hline & \multirow{4}{*}{ Logistic } & ARL & 371.67 & 305.18 & 284.01 & 191.15 & 116.23 & 72.54 & 34.44 & 16.81 & 11.03 & 3.48 & 1.98 & 1.39 & 1.08 & 1.02 \\
\hline & & MDRL & 257.50 & 201.00 & 199.00 & 129.50 & 87.00 & 52.00 & 26.00 & 13.00 & 9.00 & 3.00 & 2.00 & 1.00 & 1.00 & 1.00 \\
\hline & & SDRL & 356.53 & 305.98 & 274.70 & 187.91 & 113.63 & 70.19 & 31.77 & 13.28 & 8.81 & 1.81 & 1.07 & 0.62 & 0.30 & 0.13 \\
\hline & & ARL & 371.67 & 274.31 & 254.24 & 151.90 & 81.51 & 52.05 & 22.67 & 12.59 & 8.14 & 2.82 & 1.88 & 1.41 & 1.11 & 1.02 \\
\hline & \multirow{3}{*}{ Laplace } & MDRL & 257.50 & 191.50 & 179.00 & 112.00 & 60.00 & 36.00 & 17.00 & 10.00 & 7.00 & 3.00 & 2.00 & 1.00 & 1.00 & 1.00 \\
\hline & & SDRL & 356.53 & 269.48 & 255.88 & 148.13 & 77.52 & 49.70 & 19.76 & 10.15 & 6.24 & 1.72 & 1.01 & 0.66 & 0.35 & 0.13 \\
\hline & & ARL & 371.87 & 322.54 & 311.03 & 239.91 & 161.54 & 109.66 & 52.45 & 28.87 & 18.26 & 4.66 & 2.75 & 1.92 & 1.28 & 1.12 \\
\hline & \multirow[t]{3}{*}{$t_{4}$} & MDRL & 250.00 & 210.50 & 220.50 & 167.00 & 110.00 & 76.50 & 36.00 & 20.00 & 14.00 & 4.00 & 3.00 & 2.00 & 1.00 & 1.00 \\
\hline & & SDRL & 384.56 & 349.01 & 284.83 & 233.04 & 160.25 & 106.96 & 52.03 & 26.96 & 15.06 & 2.91 & 1.56 & 1.03 & 0.58 & 0.38 \\
\hline & & ARL & 371.82 & 320.55 & 293.03 & 225.65 & 143.39 & 95.97 & 46.46 & 24.48 & 15.52 & 4.19 & 2.37 & 1.65 & 1.18 & 1.04 \\
\hline & \multirow[t]{2}{*}{$t_{8}$} & MDRL & 260.00 & 221.00 & 203.00 & 158.00 & 103.00 & 67.00 & 34.00 & 18.00 & 12.00 & 4.00 & 2.00 & 1.00 & 1.00 & 1.00 \\
\hline & & SDRL & 367.33 & 335.03 & 302.40 & 221.66 & 139.56 & 96.20 & 42.63 & 20.99 & 12.36 & 2.51 & 1.29 & 0.82 & 0.42 & 0.20 \\
\hline
\end{tabular}

Laplace distribution is higher as compared to the other distributions used in this study (see Figures 1 and 2). The proposed control chart's ability to detect shifts deteriorates when it occurs later for small values of $\lambda(\lambda=0.05$ or $\lambda=0.10)$, whereas this trend is noticed for large values of $\lambda$ $(\lambda=0.25$ or $\lambda=0.50$ ) for large shifts (see Figures 3 and 4 ). The proposed control chart's OOC performance under imperfect RSS is also determined to illustrate the superiority of perfect RSS or generally RSS over imperfect RSS (see Tables 5-7). For example, under normal distribution, at $\lambda=0.05, n=5$, and $\delta=0.025,0.05,0.075$, and 0.10 , the ARL values of the proposed control chart under RSS are 206.77, 92.67, 50.53, and 33.9, whereas the ARL values of the proposed control chart under imperfect RSS are 295.71, 178.64, 111.43, and 73.63 (see Tables 3 and 5). In general, the proposed control chart under RSS is very sensitive to detect small and moderate shifts in the process location.
In terms of OOC performance at a specific shift $(\delta)$, the

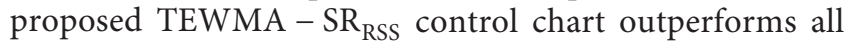
other control charts. For instance, at $\delta=0.05, \lambda=0.25$, and $n=5$, the $\mathrm{ARL}_{1}$ values of the proposed TEWMA $-\mathrm{SR}_{\mathrm{RSS}}$, TEWMA-SR, TEWMA-SN, TEWMA $-\bar{X}$, and DEWMA - $\mathrm{SR}_{\mathrm{RSS}}$ control charts are 151.84, 251.08, 341.92, 337.05 , and 167.12, respectively. The ARL is used as a metric to compare the proposed and existing control charts. Some findings are presented in the percentage decreasing in $\mathrm{ARL}=\left(\left(\mathrm{ARL}_{0}-\mathrm{ARL}_{1}\right) / \mathrm{ARL}_{0}\right) \times 100 \%$. Under normal setup, the TEWMA - $\mathrm{SR}_{\mathrm{RSS}}$ control chart displaying a 3 percent improvement in the process median minimizes the ARL by 54.56 percent for $n=5, \lambda=0.05$, and $\mathrm{ARL}_{0}=370$, whereas at the same shift $(\delta)$, the proposed control chart reduced the ARL by 80.55 percent for $n=10, \lambda=0.05$, and $\mathrm{ARL}_{0}=370$ (see Tables 3 and 4). 


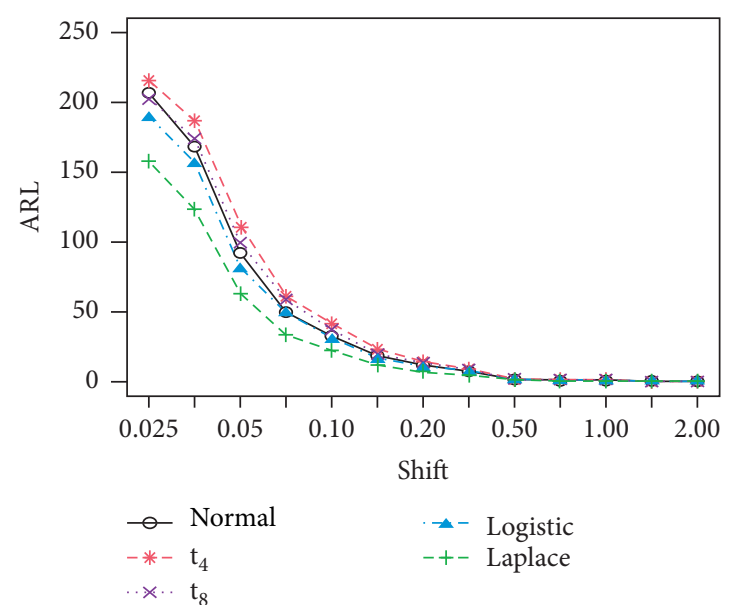

FIGURE 1: ARL behavior of various distributions using the proposed TEWMA $-\mathrm{SR}_{\mathrm{RSS}}$ control chart when $n=5, \lambda=0.05$, and $\mathrm{ARL}_{0}=370$.

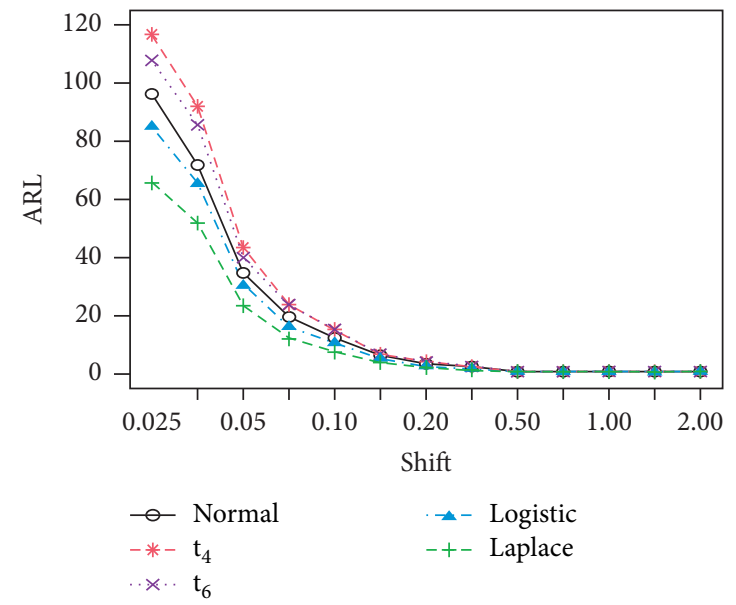

FIGURE 2: ARL behavior of various distributions using the proposed TEWMA $-\mathrm{SR}_{\mathrm{RSS}}$ control chart when $n=10, \lambda=0.05$, and $\mathrm{ARL}_{0}=370$.

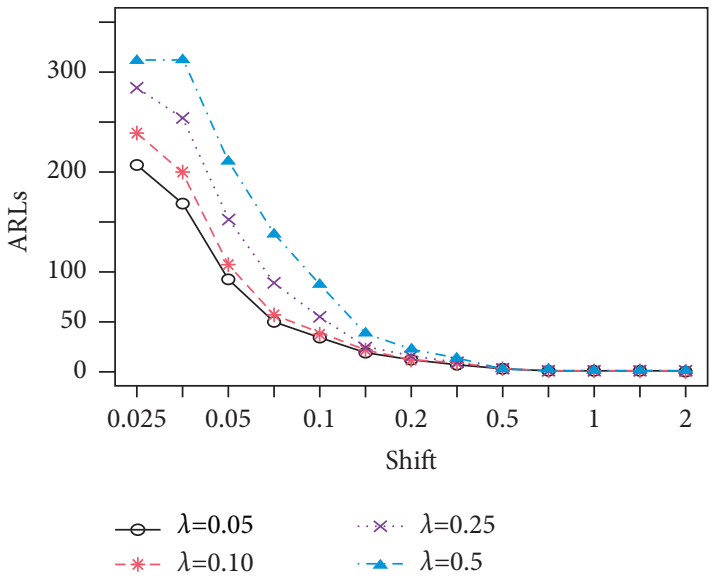

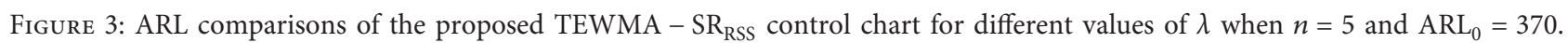




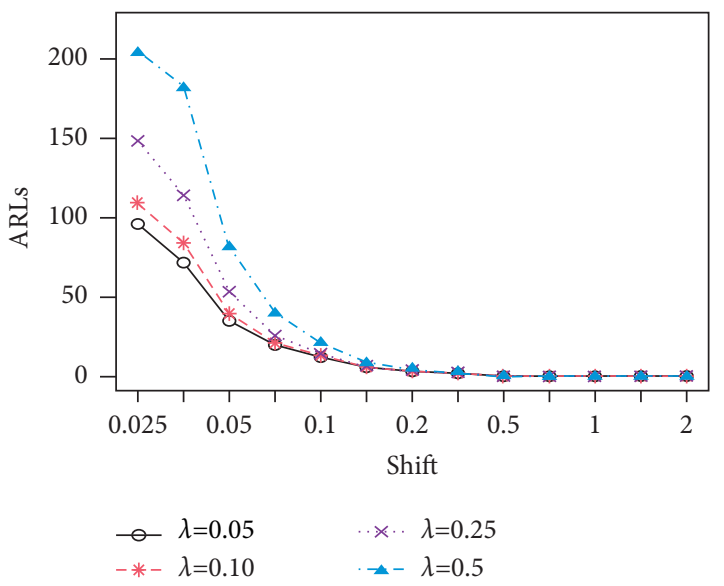

Figure 4: ARL comparisons of the proposed TEWMA - SR RSS control chart for different values of $\lambda$ when $n=10$ and $\mathrm{ARL}_{0}=370$.

TABLE 4: RL characteristics of the proposed TEWMA $-\mathrm{SR}_{\mathrm{RSS}}$ control chart under different distributions with nominal $\mathrm{ARL}_{0}=370$ and $n=10$.

\begin{tabular}{|c|c|c|c|c|c|c|c|c|c|c|c|c|c|c|c|c|}
\hline \multirow[b]{2}{*}{$(\lambda, L)$} & & & \multicolumn{14}{|c|}{$\delta$} \\
\hline & & & 0.00 & 0.025 & 0.03 & 0.05 & 0.075 & 0.10 & 0.15 & 0.20 & 0.25 & 0.50 & 0.75 & 1.00 & 1.50 & 2.00 \\
\hline \multirow{18}{*}{$(0.05,1.3635)$} & \multirow{3}{*}{$N(0,1)$} & ARL & 369.61 & 96.01 & 71.86 & 34.99 & 19.89 & 12.62 & 6.24 & 3.75 & 2.47 & 1.06 & 1.00 & 1.00 & 1.00 & 1.00 \\
\hline & & MDRL & 240.0 & 67.0 & 54.0 & 29.0 & 16.0 & 10.0 & 5.0 & 3.0 & 2.0 & 1.0 & 1.0 & 1.0 & 1.0 & 1.0 \\
\hline & & SDRL & 418.41 & 101.64 & 70.49 & 30.60 & 16.96 & 10.99 & 5.50 & 3.19 & 1.95 & 0.26 & 0.00 & 0.00 & 0.00 & 0.00 \\
\hline & \multirow{3}{*}{$\mathrm{CN}$} & ARL & 369.54 & 98.54 & 77.99 & 37.52 & 20.21 & 13.22 & 6.20 & 3.85 & 2.65 & 1.11 & 1.01 & 1.00 & 1.00 & 1.00 \\
\hline & & MDRL & 244.00 & 69.00 & 61.00 & 32.00 & 16.00 & 11.00 & 5.00 & 3.00 & 2.00 & 1.00 & 1.00 & 1.00 & 1.00 & 1.00 \\
\hline & & SDRL & 429.63 & 102.05 & 74.36 & 31.93 & 17.22 & 11.36 & 5.24 & 3.25 & 2.18 & 0.37 & 0.07 & 0.00 & 0.00 & 0.00 \\
\hline & \multirow{3}{*}{ Logistic } & ARL & 370.04 & 85.10 & 65.60 & 30.63 & 16.53 & 10.77 & 5.08 & 3.09 & 2.19 & 1.06 & 1.00 & 1.00 & 1.00 & 1.00 \\
\hline & & MDRL & 215.50 & 64.00 & 53.00 & 25.00 & 13.00 & 8.00 & 4.00 & 2.00 & 2.00 & 1.00 & 1.00 & 1.00 & 1.00 & 1.00 \\
\hline & & SDRL & 451.55 & 83.95 & 61.17 & 27.07 & 14.16 & 9.51 & 4.44 & 2.54 & 1.64 & 0.26 & 0.04 & 0.00 & 0.00 & 0.00 \\
\hline & \multirow{4}{*}{ Laplace } & ARL & 371.04 & 65.82 & 51.95 & 23.68 & 12.32 & 7.75 & 4.03 & 2.36 & 1.74 & 1.04 & 1.00 & 1.00 & 1.00 & 1.00 \\
\hline & & MDRL & 218.00 & 55.00 & 42.00 & 19.00 & 9.00 & 6.00 & 3.00 & 2.00 & 1.00 & 1.00 & 1.00 & 1.00 & 1.00 & 1.00 \\
\hline & & SDRL & 452.29 & 61.73 & 46.09 & 20.99 & 11.01 & 6.81 & 3.50 & 1.90 & 1.19 & 0.21 & 0.00 & 0.00 & 0.00 & 0.00 \\
\hline & & ARL & 370.73 & 116.79 & 91.87 & 43.49 & 24.36 & 15.90 & 7.42 & 4.85 & 3.29 & 1.23 & 1.02 & 1.00 & 1.00 & 1.00 \\
\hline & \multirow{3}{*}{$t_{4}$} & MDRL & 228.50 & 84.00 & 69.00 & 35.00 & 20.00 & 13.00 & 5.00 & 4.00 & 2.00 & 1.00 & 1.00 & 1.00 & 1.00 & 1.00 \\
\hline & & SDRL & 421.19 & 125.85 & 92.98 & 40.01 & 20.16 & 13.58 & 6.69 & 4.04 & 2.71 & 0.54 & 0.14 & 0.00 & 0.00 & 0.00 \\
\hline & & ARL & 369.67 & 107.75 & 85.82 & 40.15 & 23.71 & 14.78 & 7.08 & 4.29 & 2.87 & 1.11 & 1.01 & 1.00 & 1.00 & 1.00 \\
\hline & \multirow[t]{2}{*}{$t_{8}$} & MDRL & 225.00 & 77.00 & 65.00 & 33.00 & 20.00 & 12.00 & 5.00 & 3.00 & 2.00 & 1.00 & 1.00 & 1.00 & 1.00 & 1.00 \\
\hline & & SDRL & 441.35 & 113.15 & 83.92 & 36.90 & 18.63 & 12.45 & 6.15 & 3.58 & 2.35 & 0.39 & 0.09 & 0.00 & 0.00 & 0.00 \\
\hline \multirow{18}{*}{$(0.10,1.57)$} & \multirow{3}{*}{$N(0,1)$} & ARL & 369.43 & 109.52 & 84.48 & 39.80 & 21.08 & 13.55 & 6.82 & 4.30 & 2.87 & 1.13 & 1.00 & 1.00 & 1.00 & 1.00 \\
\hline & & MDRL & 242.50 & 76.00 & 61.00 & 33.00 & 18.00 & 12.00 & 6.00 & 4.00 & 2.00 & 1.00 & 1.00 & 1.00 & 1.00 & 1.00 \\
\hline & & SDRL & 401.47 & 109.19 & 79.78 & 32.99 & 16.75 & 10.21 & 5.30 & 3.26 & 2.14 & 0.39 & 0.04 & 0.00 & 0.00 & 0.00 \\
\hline & \multirow{3}{*}{$\mathrm{CN}$} & ARL & 369.18 & 117.33 & 91.87 & 41.32 & 23.19 & 14.61 & 7.15 & 4.58 & 3.14 & 1.17 & 1.01 & 1.00 & 1.00 & 1.00 \\
\hline & & MDRL & 244.50 & 84.00 & 68.00 & 32.50 & 21.00 & 13.00 & 6.00 & 4.00 & 3.00 & 1.00 & 1.00 & 1.00 & 1.00 & 1.00 \\
\hline & & SDRL & 401.76 & 115.00 & 87.66 & 36.27 & 16.79 & 10.75 & 5.59 & 3.48 & 2.30 & 0.46 & 0.07 & 0.00 & 0.00 & 0.00 \\
\hline & \multirow{3}{*}{ Logistic } & ARL & 369.92 & 104.66 & 73.89 & 35.34 & 19.33 & 12.30 & 6.06 & 3.73 & 2.46 & 1.09 & 1.00 & 1.00 & 1.00 & 1.00 \\
\hline & & MDRL & 243.00 & 77.00 & 54.00 & 29.50 & 17.00 & 10.00 & 5.00 & 3.00 & 2.00 & 1.00 & 1.00 & 1.00 & 1.00 & 1.00 \\
\hline & & SDRL & 419.02 & 99.93 & 71.94 & 29.90 & 14.51 & 9.21 & 4.56 & 2.78 & 1.76 & 0.33 & 0.06 & 0.00 & 0.00 & 0.00 \\
\hline & \multirow{4}{*}{ Laplace } & ARL & 369.92 & 80.30 & 57.70 & 25.08 & 13.95 & 8.93 & 4.65 & 2.88 & 2.01 & 1.08 & 1.00 & 1.00 & 1.00 & 1.00 \\
\hline & & MDRL & 243.00 & 60.00 & 45.00 & 22.00 & 12.00 & 7.50 & 4.00 & 2.00 & 1.00 & 1.00 & 1.00 & 1.00 & 1.00 & 1.00 \\
\hline & & SDRL & 419.02 & 73.03 & 52.34 & 19.71 & 10.63 & 6.76 & 3.66 & 2.16 & 1.38 & 0.29 & 0.07 & 0.00 & 0.00 & 0.00 \\
\hline & & ARL & 370.86 & 137.04 & 109.45 & 50.21 & 25.91 & 17.07 & 9.22 & 5.67 & 3.94 & 1.33 & 1.04 & 1.00 & 1.00 & 1.00 \\
\hline & \multirow[t]{3}{*}{$t_{4}$} & MDRL & 251.00 & 98.00 & 80.00 & 39.00 & 23.00 & 15.00 & 8.00 & 5.00 & 3.00 & 1.00 & 1.00 & 1.00 & 1.00 & 1.00 \\
\hline & & SDRL & 388.38 & 138.72 & 108.92 & 43.31 & 19.84 & 12.63 & 6.93 & 4.30 & 3.07 & 0.66 & 0.20 & 0.05 & 0.00 & 0.00 \\
\hline & & ARL & 370.56 & 126.76 & 101.54 & 48.45 & 23.01 & 15.84 & 8.16 & 5.04 & 3.33 & 1.21 & 1.01 & 1.00 & 1.00 & 1.00 \\
\hline & \multirow[t]{2}{*}{$t_{8}$} & MDRL & 257.50 & 87.00 & 68.50 & 38.00 & 20.00 & 14.00 & 7.00 & 4.00 & 3.00 & 1.00 & 1.00 & 1.00 & 1.00 & 1.00 \\
\hline & & SDRL & 387.66 & 130.74 & 104.01 & 41.84 & 17.88 & 11.16 & 6.14 & 3.68 & 2.39 & 0.53 & 0.10 & 0.00 & 0.00 & 0.00 \\
\hline
\end{tabular}


TABle 4: Continued.

\begin{tabular}{|c|c|c|c|c|c|c|c|c|c|c|c|c|c|c|c|c|}
\hline \multirow[b]{2}{*}{$(\lambda, L)$} & & & \multicolumn{14}{|c|}{$\delta$} \\
\hline & & & 0.00 & 0.025 & 0.03 & 0.05 & 0.075 & 0.10 & 0.15 & 0.20 & 0.25 & 0.50 & 0.75 & 1.00 & 1.50 & 2.00 \\
\hline \multirow{18}{*}{$(0.25,1.867)$} & \multirow{3}{*}{$N(0,1)$} & ARL & 370.25 & 148.30 & 114.50 & 53.87 & 26.46 & 15.29 & 7.57 & 4.94 & 3.56 & 1.25 & 1.02 & 1.00 & 1.00 & 1.00 \\
\hline & & MDRL & 271.50 & 103.00 & 78.00 & 40.00 & 20.00 & 13.00 & 7.00 & 4.00 & 3.00 & 1.00 & 1.00 & 1.00 & 1.00 & 1.00 \\
\hline & & SDRL & 353.98 & 143.90 & 113.04 & 49.08 & 22.94 & 11.38 & 5.06 & 2.97 & 2.09 & 0.52 & 0.13 & 0.00 & 0.00 & 0.00 \\
\hline & \multirow{3}{*}{$\mathrm{CN}$} & ARL & 371.05 & 154.57 & 129.10 & 59.23 & 26.81 & 16.47 & 7.95 & 5.34 & 3.66 & 1.30 & 1.02 & 1.00 & 1.00 & 1.00 \\
\hline & & MDRL & 255.50 & 105.50 & 90.00 & 44.00 & 21.00 & 14.00 & 7.00 & 5.00 & 3.00 & 1.00 & 1.00 & 1.00 & 1.00 & 1.00 \\
\hline & & SDRL & 383.00 & 152.56 & 124.95 & 56.42 & 22.54 & 12.56 & 4.99 & 3.36 & 2.09 & 0.56 & 0.14 & 0.00 & 0.00 & 0.00 \\
\hline & \multirow{3}{*}{ Logistic } & ARL & 369.80 & 142.12 & 110.97 & 45.00 & 22.01 & 13.10 & 6.86 & 4.23 & 3.03 & 1.20 & 1.01 & 1.00 & 1.00 & 1.00 \\
\hline & & MDRL & 249.00 & 97.00 & 78.00 & 32.00 & 18.00 & 11.00 & 6.00 & 4.00 & 3.00 & 1.00 & 1.00 & 1.00 & 1.00 & 1.00 \\
\hline & & SDRL & 402.95 & 147.38 & 102.81 & 41.45 & 16.83 & 9.21 & 4.22 & 2.72 & 1.85 & 0.46 & 0.09 & 0.03 & 0.00 & 0.00 \\
\hline & \multirow{3}{*}{ Laplace } & ARL & 369.80 & 111.92 & 78.38 & 33.46 & 16.67 & 9.76 & 5.28 & 3.30 & 2.50 & 1.14 & 1.02 & 1.00 & 1.00 & 1.00 \\
\hline & & MDRL & 249.00 & 81.00 & 54.00 & 26.00 & 14.00 & 9.00 & 5.00 & 3.00 & 2.00 & 1.00 & 1.00 & 1.00 & 1.00 & 1.00 \\
\hline & & SDRL & 402.95 & 105.95 & 75.98 & 28.81 & 12.94 & 6.80 & 3.23 & 2.04 & 1.50 & 0.37 & 0.14 & 0.00 & 0.00 & 0.00 \\
\hline & \multirow{3}{*}{$t_{4}$} & ARL & 368.97 & 186.35 & 140.23 & 71.38 & 34.86 & 19.43 & 9.78 & 6.19 & 4.22 & 1.56 & 1.09 & 1.01 & 1.00 & 1.00 \\
\hline & & MDRL & 262.00 & 131.00 & 97.50 & 50.00 & 26.00 & 16.00 & 9.00 & 6.00 & 4.00 & 1.00 & 1.00 & 1.00 & 1.00 & 1.00 \\
\hline & & SDRL & 359.24 & 181.23 & 140.44 & 67.16 & 29.67 & 14.58 & 6.51 & 4.05 & 2.63 & 0.79 & 0.30 & 0.11 & 0.00 & 0.00 \\
\hline & \multirow{3}{*}{$t_{8}$} & ARL & 371.13 & 177.20 & 148.49 & 62.09 & 30.50 & 17.80 & 9.01 & 5.81 & 3.75 & 1.41 & 1.04 & 1.00 & 1.00 & 1.00 \\
\hline & & MDRL & 259.00 & 125.00 & 101.50 & 44.50 & 23.00 & 15.00 & 8.00 & 5.00 & 3.00 & 1.00 & 1.00 & 1.00 & 1.00 & 1.00 \\
\hline & & SDRL & 367.57 & 173.08 & 155.73 & 54.46 & 25.64 & 13.55 & 5.73 & 3.45 & 2.21 & 0.69 & 0.20 & 0.05 & 0.00 & 0.00 \\
\hline \multirow{18}{*}{$(0.50,2.1185)$} & \multirow{3}{*}{$N(0,1)$} & ARL & 370.25 & 203.92 & 181.53 & 81.69 & 39.73 & 21.85 & 8.99 & 5.16 & 3.64 & 1.35 & 1.02 & 1.00 & 1.00 & 1.00 \\
\hline & & MDRL & 261.00 & 144.00 & 131.50 & 55.00 & 29.00 & 16.00 & 7.00 & 5.00 & 3.00 & 1.00 & 1.00 & 1.00 & 1.00 & 1.00 \\
\hline & & SDRL & 361.13 & 203.76 & 174.27 & 81.56 & 36.79 & 19.27 & 6.49 & 3.35 & 2.03 & 0.56 & 0.15 & 0.00 & 0.00 & 0.00 \\
\hline & \multirow{3}{*}{$\mathrm{CN}$} & ARL & 371.05 & 220.20 & 176.01 & 89.51 & 45.28 & 23.66 & 9.17 & 5.40 & 3.82 & 1.40 & 1.05 & 1.00 & 1.00 & 1.00 \\
\hline & & MDRL & 266.50 & 144.00 & 129.50 & 63.00 & 33.00 & 17.00 & 7.00 & 5.00 & 3.00 & 1.00 & 1.00 & 1.00 & 1.00 & 1.00 \\
\hline & & SDRL & 355.54 & 224.64 & 167.97 & 86.11 & 44.09 & 20.66 & 6.81 & 3.40 & 2.19 & 0.59 & 0.21 & 0.04 & 0.00 & 0.00 \\
\hline & \multirow{3}{*}{ Logistic } & ARL & 369.71 & 207.36 & 144.94 & 72.68 & 33.57 & 18.75 & 7.77 & 4.62 & 3.28 & 1.26 & 1.01 & 1.00 & 1.00 & 1.00 \\
\hline & & MDRL & 262.00 & 140.50 & 107.50 & 53.00 & 25.00 & 15.00 & 7.00 & 4.00 & 3.00 & 1.00 & 1.00 & 1.00 & 1.00 & 1.00 \\
\hline & & SDRL & 389.82 & 220.88 & 139.79 & 69.42 & 31.08 & 15.69 & 5.48 & 2.89 & 1.81 & 0.49 & 0.12 & 0.04 & 0.00 & 0.00 \\
\hline & & ARL & 369.71 & 165.97 & 118.11 & 47.01 & 21.84 & 12.14 & 5.65 & 3.50 & 2.55 & 1.22 & 1.02 & 1.00 & 1.00 & 1.00 \\
\hline & \multirow[t]{3}{*}{ Laplace } & MDRL & 262.00 & 112.00 & 81.50 & 33.50 & 16.00 & 9.00 & 5.00 & 3.00 & 2.00 & 1.00 & 1.00 & 1.00 & 1.00 & 1.00 \\
\hline & & SDRL & 389.82 & 177.25 & 114.36 & 42.64 & 19.12 & 9.66 & 3.58 & 2.00 & 1.38 & 0.45 & 0.14 & 0.05 & 0.00 & 0.00 \\
\hline & & ARL & 369.17 & 242.08 & 202.82 & 104.20 & 55.12 & 29.44 & 12.56 & 7.09 & 4.64 & 1.64 & 1.12 & 1.03 & 1.00 & 1.00 \\
\hline & & MDRL & 261.50 & 172.00 & 141.00 & 76.00 & 38.00 & 22.00 & 9.00 & 6.00 & 4.00 & 1.00 & 1.00 & 1.00 & 1.00 & 1.00 \\
\hline & & SDRL & 359.03 & 241.26 & 199.81 & 101.71 & 54.08 & 26.61 & 10.82 & 4.73 & 2.73 & 0.79 & 0.34 & 0.16 & 0.04 & 0.00 \\
\hline & & ARL & 369.74 & 240.61 & 203.22 & 99.49 & 48.13 & 26.55 & 10.96 & 5.74 & 4.13 & 1.56 & 1.06 & 1.01 & 1.00 & 1.00 \\
\hline & \multirow[t]{2}{*}{$t_{8}$} & MDRL & 253.00 & 173.00 & 138.00 & 67.00 & 34.50 & 20.00 & 9.00 & 5.00 & 4.00 & 1.00 & 1.00 & 1.00 & 1.00 & 1.00 \\
\hline & & SDRL & 366.34 & 229.90 & 209.30 & 100.88 & 44.50 & 24.28 & 8.71 & 3.80 & 2.32 & 0.74 & 0.24 & 0.08 & 0.00 & 0.00 \\
\hline
\end{tabular}

\section{Comparative Study}

This section provides the proposed control chart's comparisons to several existing control charts, which includes

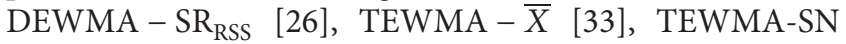
[32], and TEWMA-SR [4] control charts. More detail is provided in the following sections.

4.1. Proposed versus TEWMA-SR Control Chart. The pro-

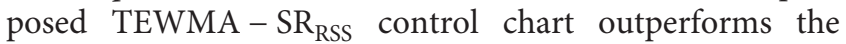
TEWMA-SR control chart. For instance, at $n=5$ and $\lambda=$ 0.05 with $\delta=5$ percent shift in the Logistic distribution, the proposed TEWMA - SR RSS control chart reduces the $A R L_{1}$ value by almost 78.28 percent, whereas the TEWMA-SR control chart reduces the $\mathrm{ARL}_{1}$ value by 58.74 percent (see Table 8). Similarly, under $t_{(4)}$ distribution, when $n=10$ and $\lambda=0.10$ along with $\delta=5$ percent, the $\mathrm{ARL}_{1}$ values of the proposed TEWMA - SR $R_{R S S}$ control chart are reduced by 86.46 percent, while the TEWMA-SR control chart reduces the values by 72.30 percent (see Tables 4 and 9). Figures 5 and 6 highlight the proposed TEWMA - SR $\mathrm{RSS}_{\text {control chart }}$ superiority over the TEWMA-SR control chart. It is worth mentioning that, for small shifts, the proposed TEWMA - $\mathrm{SR}_{\mathrm{RSS}}$ control chart outperforms the TEWMASR control chart for different combinations of $\lambda$ and $n$.

4.2. Proposed versus TEWMA-SN Control Chart. The RL features of the TEWMA-SN control chart for the process location are listed in Table 10. The proposed TEWMA - $\mathrm{SR}_{\mathrm{RSS}}$ control chart is more efficient than the TEWMA-SN control chart. For example, under Laplace distribution, the proposed TEWMA $-\mathrm{SR}_{\mathrm{RSS}}$ control chart with $n=10$ and $\lambda=0.05$ with $\delta=10$ percent shows OOC situation almost after 7.75 observations, whereas the TEWMA-SN control chart gives OOC state nearly after 25.67 observations (see Tables 4 and 10). Similarly, in the $t_{(8)}$ distribution scenario, at $n=10$ and $\lambda=0.05$ with a shift of 5 percent, the TEWMA-SN control chart decreases the ARL 
TABLE 5: ARL measures of the proposed control chart with imperfect ranked set sampling under normal distribution at $n=5$ and $\mathrm{ARL}_{0}=370$.

\begin{tabular}{|c|c|c|c|c|c|c|c|c|c|c|c|c|c|c|c|c|}
\hline \multirow[b]{2}{*}{$\lambda, L$} & \multirow[b]{2}{*}{$\gamma$} & \multicolumn{15}{|c|}{$\delta$} \\
\hline & & & 0 & 0.025 & 0.03 & 0.05 & 0.075 & 0.1 & 0.15 & 0.2 & .25 & 0.5 & 0.75 & 1 & 1.5 & 2 \\
\hline \multirow{12}{*}{$0.05,1.793$} & \multirow{3}{*}{0.30} & ARL & 73.21 & 95.71 & 74.87 & 178.64 & 111.43 & 73.63 & 9.94 & 24.53 & 8.50 & 6.03 & 2.97 & 2.02 & 1.34 & 1.12 \\
\hline & & MDRL & 242.50 & 192.50 & & & 80.50 & 59.00 & 33.00 & 20.00 & & 5.00 & 2.00 & 2.00 & 1.00 & 1.00 \\
\hline & & SDRL & 437.59 & 321.50 & 293.65 & 193.69 & 114.14 & 71.00 & 35.36 & 20.83 & 15.18 & 5.01 & 2.31 & 1.28 & 0.56 & 0.32 \\
\hline & \multirow{3}{*}{0.50} & ARL & 370.86 & 282.37 & 265.80 & 154.96 & 101.64 & 67.17 & 37.90 & 23.53 & 16.46 & 5.17 & 2.63 & .66 & 1.13 & 1.02 \\
\hline & & MDRL & 218.50 & 174.50 & 158.50 & 106.50 & 73.00 & 53.00 & 32.50 & 20.00 & & 4.00 & 2.00 & 1.00 & 1.00 & 1.00 \\
\hline & & SDRL & 454.57 & 327.88 & 315.97 & 169.82 & 104.79 & 64.61 & 32.58 & 19.65 & 14.33 & 4.48 & 2.19 & 1.13 & 0.40 & 0.16 \\
\hline & \multirow{3}{*}{0.70} & ARL & 372.89 & 266.56 & 243.76 & 140.66 & 88.90 & 59.96 & 33.18 & 19.77 & 15.02 & 4.54 & 2.29 & 1.59 & 1.09 & \\
\hline & & MDRL & 242.00 & 169.50 & 153.50 & 95.50 & 66.00 & 49.00 & 27.00 & 16.00 & & 4.00 & 2.00 & 1.00 & 1.00 & 1.00 \\
\hline & & SDRL & 452.69 & 294.47 & 298.29 & 155.58 & & 55.61 & 28.46 & 17.16 & & 3.71 & 1.73 & 0.98 & 0.32 & 0.09 \\
\hline & \multirow{3}{*}{0.90} & ARL & 372.08 & 243.06 & 209.25 & 121.65 & 66.33 & 44.54 & 24.92 & 16.12 & & 3.48 & 1.83 & .32 & 04 & 1.00 \\
\hline & & MDRL & 228.00 & 160.00 & 138.50 & 84.50 & 48.00 & 36.00 & 20.00 & 13.00 & 9.00 & 3.00 & 1.00 & 1.00 & 1.00 & 1.00 \\
\hline & & SDRL & 459.42 & 272.24 & 231.19 & 127.67 & 64.01 & 38.87 & 21.67 & 13.48 & (.00 & 2.78 & 1.19 & 0.63 & 0.19 & 0.04 \\
\hline \multirow{12}{*}{$0.1,2.069$} & \multirow{3}{*}{0.30} & $\mathrm{I}$ & 2 & 5 & 1 & 3 & 1 & 81.36 & .55 & 37 & & 7.28 & 5 & 73 & 9 & 2.01 \\
\hline & & MDRL & & 21 & 21 & & & 59.00 & & & & 6.00 & 3.00 & 2.00 & 2.00 & 2.00 \\
\hline & & SDRL & 405.22 & 325.15 & 300.95 & 227.28 & 122 & 76.22 & 39.13 & & & 4.85 & 2.35 & 1.24 & 0.36 & 0.11 \\
\hline & \multirow{3}{*}{0.50} & ARL & 372.77 & 292.15 & 277.20 & 186.86 & 116.44 & 76.80 & 40.35 & 26.47 & & 6.37 & 3.23 & 2.20 & & 1.13 \\
\hline & & MDRL & 242.50 & 199.00 & 184.50 & 129.00 & 84.00 & 57.00 & 34.50 & 0 & & 6.00 & 3.00 & 0 & 1.00 & .00 \\
\hline & & SDRL & 421.10 & 321.22 & 303 & 190.83 & 1 & 72.90 & 32.48 & 89 & & 4.44 & 2.20 & 1.38 & 0.66 & .35 \\
\hline & \multirow{4}{*}{0.70} & ARL & 368.36 & 291.14 & 266.67 & 170.19 & 111.43 & 63.57 & 36.22 & 21.48 & & 5.39 & 2.86 & 2.01 & 1.34 & 1.11 \\
\hline & & MDRL & 239.50 & 194.00 & 180.00 & 116.50 & 80.00 & 50.00 & 31.00 & 19.00 & 14.00 & 5.00 & 2.00 & 2.00 & 1.00 & 1.00 \\
\hline & & SDRL & & 310.09 & & & & 56.54 & 28.78 & & & 3.85 & & & & \\
\hline & & & 37 & 266 & & 13 & & 53.27 & 27.15 & & & 4.01 & 2.14 & 1.52 & 9 & .01 \\
\hline & \multirow[t]{2}{*}{0.90} & MDRL & 245.00 & 171.00 & 163.00 & 99.00 & 59.00 & 42.00 & 23.00 & 16.00 & 10.00 & 3.00 & 2.00 & 1.00 & 1.00 & 1.00 \\
\hline & & SDRL & 383.69 & 280.70 & 257.04 & 138.71 & 71.74 & 44.77 & 21.03 & 12.92 & 9.13 & 2.85 & 1.36 & 0.75 & 0.29 & 0.11 \\
\hline \multirow{12}{*}{$0.25,2.045$} & \multirow{4}{*}{0.30} & 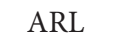 & 2.80 & 44.43 & 25.65 & 610 & 68.50 & 121.69 & 1020 & $20-0$ & & 7.79 & 1 & 2 & 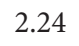 & 2.03 \\
\hline & & MDRL & 25 & & 236 & & & 50 & & 0 & & 7.00 & & & & 2.00 \\
\hline & & SDRL & & & & & & & & & & & & & & \\
\hline & & ARL & 371.94 & 337 & 304 & 23 & 15 & 107.13 & 56.45 & 33.32 & & 7.05 & 4.15 & 2.95 & 2.16 & 2.02 \\
\hline & \multirow[t]{3}{*}{0.50} & MDRL & 249.50 & 240.50 & 196.50 & 156.50 & 114.00 & 73.00 & 41.00 & 26.00 & & 6.00 & 4.00 & 3.00 & 2.00 & 2.00 \\
\hline & & SDRL & 378.92 & 325.82 & 317.83 & 241.56 & 150.45 & 106.15 & 53.87 & 28.04 & & 3.98 & 2.02 & 1.19 & 0.42 & 0.13 \\
\hline & & & 372.26 & 333.52 & 317.06 & 227.03 & 147 & 95.56 & 49.19 & 27.66 & & 6.12 & & & 2.07 & 2.00 \\
\hline & 0.70 & MDRL & 247.00 & 232.50 & 207.50 & 153.00 & 99.00 & 70.00 & 36.00 & 21.00 & 16.00 & 6.00 & 3.00 & 2.00 & 2.00 & 2.00 \\
\hline & & SDRL & 389.33 & 325.75 & 346.54 & 235.50 & 152.82 & 88.01 & 43.38 & 22.61 & 13.86 & 3.41 & 1.69 & 0.87 & 0.29 & 0.04 \\
\hline & & & 368.96 & 319.23 & 277.69 & & 114.74 & 70.32 & 34.78 & & & 4.63 & 2.68 & 1.90 & & 1.11 \\
\hline & 0.90 & MDRL & & & & & & 51.00 & 27.00 & 16 & 12.00 & 4.00 & 2.00 & 2.00 & 1.00 & 1.00 \\
\hline & & SDRL & 377.52 & 323.88 & 280.01 & 183.71 & 114.77 & 63.45 & 29.24 & 14.78 & 9.94 & 2.60 & 1.44 & 0.84 & 0.48 & 0.31 \\
\hline & & 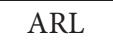 & 3 & 3 & 8 & 9 & 2 & 16 & 94 & 5 & & 4 & & & 2.55 & 23 \\
\hline & 0.30 & MDRL & 255.00 & 220.00 & 237.00 & 210.50 & 158.00 & 113.00 & 68.50 & 42.00 & 27.00 & 8.00 & 4.00 & 3.00 & 2.00 & 2.00 \\
\hline & & SDRL & 363.45 & 345.01 & 342.11 & 276.99 & 238.32 & 183.43 & 87.73 & 54.94 & & 6.50 & 2.09 & 1.23 & 0.60 & 0.42 \\
\hline & & & 368.41 & & & & & 154.20 & 83.56 & & & 8.34 & 4.35 & 3.06 & 2.25 & 2.05 \\
\hline & 0.50 & MDRL & 253.50 & 237.00 & 220.50 & 198.00 & 147.00 & 111.00 & 57.00 & 35.00 & & 7.00 & 4.00 & 3.00 & 2.00 & 2.00 \\
\hline & & SDRL & 351.77 & 342.73 & 305.48 & 265.20 & 208.49 & 153.40 & 82.90 & 50.55 & 31.97 & 5.64 & 2.04 & 1.10 & 0.46 & 0.22 \\
\hline & & ARL & 370.94 & 364.34 & 336.00 & 280.24 & 203.91 & 152.09 & 76.83 & 45.71 & 28.22 & 6.93 & 3.83 & 2.84 & 2.16 & 2.02 \\
\hline & 0.70 & MDRL & 246.50 & 273.50 & 234.00 & 193.50 & 144.50 & 102.00 & 54.50 & 35.00 & 21.00 & 6.00 & 4.00 & 3.00 & 2.00 & 2.00 \\
\hline & & SDRL & 378.51 & 357.52 & 335.50 & 276.70 & 200.57 & 156.78 & 76.09 & 39.61 & 25.80 & 4.37 & 1.58 & 0.90 & 0.39 & 0.15 \\
\hline & & ARL & 369.05 & 349.47 & 318.40 & 251.53 & 170.83 & 111.83 & 55.24 & 32.59 & 18.51 & 5.09 & 2.97 & 2.34 & 2.02 & 2.00 \\
\hline & 0.90 & MDRL & 271.00 & 260.00 & 221.00 & 173.00 & 126.00 & 78.00 & 38.50 & 24.00 & 14.00 & 5.00 & 3.00 & 2.00 & 2.00 & 2.00 \\
\hline & & SDRL & 354.84 & 328.59 & 314.95 & 247.96 & 161.02 & 106.93 & 51.79 & 30.19 & 15.07 & 2.72 & 1.13 & 0.63 & 0.15 & 0.03 \\
\hline
\end{tabular}

by 67.83 percent, while the proposed TEWMA-SR $\mathrm{RSS}$ control chart decreases the $\mathrm{ARL}_{1}$ by 89.13 percent (see Table 8).

Furthermore, at $n=5$ and $\lambda=0.05$ with 10 percent shift in process location under the Logistic distribution, the TEWMA-SN control chart reduces the $\mathrm{ARL}_{1}$ by 79.95 percent and the proposed TEWMA - $\mathrm{SR}_{\mathrm{RSS}}$ control chart reduces the $\mathrm{ARL}_{1}$ by 91.79 percent (see Table 8 ). The pro-

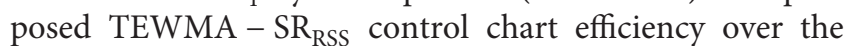
TEWMA-SN control chart can be seen in Figure 6. In short, the proposed TEWMA - $\mathrm{SR}_{\mathrm{RSS}}$ control chart is quite sensitive under normal and non-normal distributions for detecting small to moderate shifts in process location against the TEWMA-SN control chart. 
TABLE 6: ARL measures of the proposed control chart with imperfect ranked set sampling under normal distribution at $n=10$ and $\mathrm{ARL}_{0}=370$.

\begin{tabular}{|c|c|c|c|c|c|c|c|c|c|c|c|c|c|c|c|c|}
\hline \multirow[b]{2}{*}{$\lambda, L$} & \multirow[b]{2}{*}{$\gamma$} & & \multicolumn{14}{|c|}{$\delta$} \\
\hline & & & 0 & 0.025 & 0.03 & 0.05 & 0.075 & 0.1 & 0.15 & 0.2 & 0.25 & 0.5 & 0.75 & 1 & 1.5 & 2 \\
\hline \multirow{12}{*}{$0.05,1.76$} & \multirow{3}{*}{0.30} & ARL & 373.11 & 218.89 & 192.52 & 99.22 & 60.61 & 39.78 & 20.64 & 13.46 & 9.84 & 2.93 & 1.56 & 1.17 & 1.01 & 1.00 \\
\hline & & MDRL & 204.50 & 146.00 & 131.00 & 78.00 & 48.00 & 34.00 & 16.00 & 10.50 & 8.00 & 2.00 & 1.00 & 1.00 & 1.00 & 1.00 \\
\hline & & SDRL & 454.49 & 241.25 & 198.80 & 97.04 & 57.92 & 33.97 & 18.16 & 11.81 & 8.36 & 2.44 & 1.00 & 0.44 & 0.09 & 0.00 \\
\hline & \multirow{4}{*}{0.50} & ARL & 373.21 & 207.83 & 168.22 & 98.24 & 57.07 & 37.64 & 19.48 & 12.86 & 8.89 & 2.62 & 1.38 & 1.12 & 1.00 & 1.00 \\
\hline & & MDRL & 216.50 & 128.00 & 112.00 & 75.50 & 48.00 & 30.00 & 16.00 & 9.00 & 7.00 & 2.00 & 1.00 & 1.00 & 1.00 & 1.00 \\
\hline & & SDRL & 477.82 & 243.21 & 178.19 & 100.55 & 50.78 & 33.88 & 16.65 & 11.20 & 7.60 & 2.07 & 0.81 & 0.38 & 0.05 & 0.00 \\
\hline & & ARL & 372.35 & 178.19 & 155.38 & 76.48 & 45.13 & 29.63 & 16.62 & 10.07 & 6.78 & 2.01 & 1.27 & 1.04 & 1.00 & 1.00 \\
\hline & \multirow[t]{3}{*}{0.70} & MDRL & 231.00 & 118.00 & 105.50 & 59.00 & 37.00 & 24.50 & 13.00 & 8.00 & 5.00 & 1.00 & 1.00 & 1.00 & 1.00 & 1.00 \\
\hline & & SDRL & 454.56 & 199.24 & 164.83 & 75.30 & 39.18 & 25.75 & 14.87 & 8.91 & 5.75 & 1.48 & 0.63 & 0.19 & 0.00 & 0.00 \\
\hline & & ARL & 374.41 & 130.95 & 112.56 & 55.23 & 30.99 & 20.17 & 10.80 & 6.54 & 3.99 & 1.43 & 1.04 & 1.00 & 1.00 & 1.00 \\
\hline & \multirow{2}{*}{0.90} & MDRL & 255.00 & 93.50 & 86.50 & 44.00 & 25.00 & 17.00 & 9.00 & 5.00 & 3.00 & 1.00 & 1.00 & 1.00 & 1.00 & 1.00 \\
\hline & & SDRL & 430.14 & 142.14 & 109.65 & 50.66 & 27.54 & 16.64 & 8.99 & 5.68 & 3.47 & 0.79 & 0.19 & 0.05 & 0.00 & 0.00 \\
\hline \multirow{12}{*}{$0.10,2.045$} & \multirow{3}{*}{0.30} & ARL & 371.60 & 251.30 & 203.89 & 117.69 & 67.67 & 44.89 & 22.92 & 15.19 & 10.75 & 3.55 & 1.82 & 1.24 & 1.01 & 1.00 \\
\hline & & MDRL & 234.50 & 180.50 & 142.50 & 86.00 & 49.00 & 37.00 & 20.00 & 13.50 & 10.00 & 3.00 & 1.00 & 1.00 & 1.00 & 1.00 \\
\hline & & SDRL & 414.94 & 261.17 & 210.13 & 124.04 & 61.25 & 37.37 & 17.87 & 11.19 & 8.04 & 2.54 & 1.14 & 0.56 & 0.09 & 0.03 \\
\hline & \multirow{4}{*}{0.50} & ARL & 368.71 & 236.57 & 205.49 & 118.96 & 64.65 & 40.98 & 21.86 & 14.36 & 9.63 & 3.09 & 1.67 & 1.17 & 1.01 & 1.00 \\
\hline & & MDRL & 236.00 & 152.00 & 146.50 & 82.50 & 50.00 & 35.00 & 19.00 & 13.00 & 9.00 & 2.00 & 1.00 & 1.00 & 1.00 & 1.00 \\
\hline & & SDRL & 395.36 & 259.02 & 207.69 & 120.22 & 57.53 & 34.11 & 17.06 & 10.42 & 7.00 & 2.29 & 1.04 & 0.44 & 0.07 & 0.03 \\
\hline & & ARL & 369.42 & 220.71 & 182.66 & 92.77 & 51.60 & 33.85 & 17.38 & 11.20 & 7.96 & 2.46 & 1.37 & 1.08 & 1.00 & 1.00 \\
\hline & \multirow[t]{3}{*}{0.70} & MDRL & 249.00 & 157.00 & 122.00 & 68.00 & 41.00 & 28.00 & 15.00 & 10.00 & 7.00 & 2.00 & 1.00 & 1.00 & 1.00 & 1.00 \\
\hline & & SDRL & 380.61 & 230.25 & 186.90 & 85.07 & 44.65 & 29.42 & 12.75 & 8.39 & 6.01 & 1.75 & 0.72 & 0.30 & 0.05 & 0.00 \\
\hline & & ARL & 368.55 & 154.11 & 128.57 & 63.30 & 34.41 & 21.62 & 11.69 & 7.51 & 4.95 & 1.54 & 1.07 & 1.01 & 1.00 & 1.00 \\
\hline & \multirow[t]{2}{*}{0.90} & MDRL & 244.50 & 102.00 & 97.50 & 49.00 & 28.00 & 18.00 & 10.00 & 7.00 & 4.00 & 1.00 & 1.00 & 1.00 & 1.00 & 1.00 \\
\hline & & SDRL & 376.02 & 169.42 & 124.19 & 57.57 & 27.88 & 16.53 & 9.13 & 5.29 & 3.81 & 0.92 & 0.31 & 0.09 & 0.00 & 0.00 \\
\hline \multirow{12}{*}{$0.25,2.445$} & \multirow{3}{*}{0.30} & ARL & 368.15 & 291.14 & 249.72 & 167.77 & 103.44 & 64.41 & 31.10 & 19.14 & 12.31 & 4.27 & 2.32 & 1.60 & 1.09 & 1.01 \\
\hline & & MDRL & 257.50 & 201.00 & 176.50 & 118.00 & 72.00 & 48.00 & 24.00 & 16.00 & 11.00 & 4.00 & 2.00 & 1.00 & 1.00 & 1.00 \\
\hline & & SDRL & 368.63 & 304.07 & 269.34 & 162.29 & 100.22 & 58.65 & 25.50 & 14.84 & 8.82 & 2.41 & 1.23 & 0.76 & 0.29 & 0.09 \\
\hline & \multirow{4}{*}{0.50} & ARL & 371.23 & 299.87 & 272.71 & 172.55 & 100.00 & 59.62 & 28.46 & 15.83 & 11.11 & 3.88 & 2.09 & 1.47 & 1.05 & 1.00 \\
\hline & & MDRL & 260.00 & 210.00 & 179.00 & 131.00 & 73.00 & 41.00 & 22.00 & 13.00 & 10.00 & 3.00 & 2.00 & 1.00 & 1.00 & 1.00 \\
\hline & & SDRL & 364.66 & 296.55 & 284.84 & 157.21 & 93.60 & 57.66 & 22.18 & 11.10 & 7.06 & 2.21 & 1.10 & 0.61 & 0.22 & 0.04 \\
\hline & & ARL & 373.52 & 261.53 & 224.48 & 128.57 & 74.51 & 46.11 & 21.39 & 12.85 & 8.93 & 2.89 & 1.68 & 1.22 & 1.00 & 1.00 \\
\hline & 0.70 & MDRL & 256.00 & 187.50 & 152.00 & 93.00 & 53.00 & 33.00 & 17.00 & 11.00 & 8.00 & 3.00 & 1.00 & 1.00 & 1.00 & 1.00 \\
\hline & & SDRL & 373.99 & 261.14 & 230.15 & 125.34 & 69.38 & 42.72 & 17.55 & 8.42 & 5.75 & 1.64 & 0.82 & 0.47 & 0.05 & 0.00 \\
\hline & & ARL & 368.26 & 204.11 & 178.21 & 88.57 & 45.60 & 25.69 & 13.01 & 8.01 & 5.78 & 1.95 & 1.22 & 1.03 & 1.00 & 1.00 \\
\hline & 0.90 & MDRL & 244.50 & 147.50 & 132.50 & 62.00 & 33.00 & 19.00 & 11.00 & 7.00 & 5.00 & 2.00 & 1.00 & 1.00 & 1.00 & 1.00 \\
\hline & & SDRL & 372.36 & 199.39 & 164.41 & 87.90 & 41.70 & 22.39 & 9.14 & 5.09 & 3.45 & 1.06 & 0.47 & 0.16 & 0.00 & 0.00 \\
\hline & & ARL & 371.28 & 308.77 & 280.73 & 225.25 & 146.06 & 101.05 & 46.52 & 25 & 16.15 & 4.49 & 2.53 & 1.91 & 1.32 & 1.06 \\
\hline & 0.30 & MDRL & 272.50 & 226.50 & 205.00 & 153.00 & 106.00 & 67.00 & 32.00 & 18.00 & 12.00 & 4.00 & 2.00 & 2.00 & 1.00 & 1.00 \\
\hline & & SDRL & 340.94 & 288.19 & 268.66 & 217.69 & 139.07 & 100.39 & 41.57 & 22.77 & 13.14 & 2.43 & 1.05 & 0.71 & 0.47 & 0.24 \\
\hline & & ARL & 369.52 & 329.86 & 318.54 & 231.50 & 150.59 & 88.17 & 45.15 & 23.44 & 14.67 & 3.97 & 2.31 & 1.75 & 1.23 & 1.03 \\
\hline & 0.50 & MDRL & 248.00 & 231.50 & 213.50 & 164.00 & 117.00 & 61.00 & 33.00 & 17.00 & 11.00 & 4.00 & 2.00 & 2.00 & 1.00 & 1.00 \\
\hline & & SDRL & 368.35 & 328.72 & 329.20 & 220.58 & 138.50 & 89.27 & 41.23 & 19.84 & 12.32 & 2.02 & 0.95 & 0.61 & 0.42 & 0.17 \\
\hline & & ARL & 367.86 & 299.83 & 271.13 & 188.83 & 122.42 & 76.20 & 31.64 & 17.58 & 11.07 & 3.28 & 1.95 & 1.40 & 1.05 & 1.00 \\
\hline & 0.70 & MDRL & 243.00 & 213.50 & 176.50 & 131.00 & 87.00 & 54.00 & 23.00 & 14.00 & 9.00 & 3.00 & 2.00 & 1.00 & 1.00 & 1.00 \\
\hline & & SDRL & 333.88 & 292.66 & 272.78 & 192.12 & 119.05 & 73.75 & 28.33 & 14.71 & 8.81 & 1.67 & 0.79 & 0.55 & 0.21 & 0.03 \\
\hline & & ARL & 367.95 & 247.37 & 232.26 & 139.19 & 73.17 & 40.68 & 17.75 & 9.36 & 6.10 & 2.15 & 1.32 & 1.05 & 1.00 & 1.00 \\
\hline & 0.90 & MDRL & 261.50 & 180.00 & 165.50 & 94.00 & 51.00 & 29.00 & 13.00 & 7.00 & 5.00 & 2.00 & 1.00 & 1.00 & 1.00 & 1.00 \\
\hline & & SDRL & 356.50 & 243.31 & 219.41 & 138.17 & 72.32 & 37.53 & 14.59 & 7.17 & 3.93 & 0.99 & 0.51 & 0.21 & 0.00 & 0.00 \\
\hline
\end{tabular}

4.3. Proposed versus TEWMA- $\bar{X}$ Control Chart. The TEWMA - $\mathrm{SR}_{\mathrm{RSS}}$ control chart outperforms the TEWMA $\bar{X}$ control chart in terms of early shift detection ability. For instance, using the Laplace distribution, the TEWMA $-\bar{X}$ control chart with $n=5$ and $\lambda=0.25$ for $\delta=5$ percent gives OOC signal after 238.73 observations, whereas the proposed TEWMA - $\mathrm{SR}_{\mathrm{RSS}}$ control chart shows the OOC signal after 105.76 observations (see Table 8 ). The superiority of the proposed control chart can also be seen in the case of outliers under $\mathrm{CN}$ distribution. For example, the proposed TEWMA $-\mathrm{SR}_{\mathrm{RSS}}$ control chart with $n=10$ and $\lambda=0.10$ for $\delta=10$ percent produces OOC signal after 14.61 observations, while the TEWMA $-\bar{X}$ control chart shows OOC signal after 43.39 observations (see Tables 4 and 11). The performance of the proposed control chart over the TEWMA $-\bar{X}$ control chart is depicted in Figures 7 and 8. In 
TABLE 7: ARL measures of the proposed control chart with imperfect ranked set sampling under $t$-distribution $(\mathrm{d} f=4)$ at $n=5$ and $\mathrm{ARL}_{0}=370$.

\begin{tabular}{|c|c|c|c|c|c|c|c|c|c|c|c|c|c|c|c|c|}
\hline \multirow[b]{2}{*}{$\lambda, L$} & \multirow[b]{2}{*}{$\gamma$} & & \multicolumn{14}{|c|}{$\delta$} \\
\hline & & & 0 & 0.025 & 0.03 & 0.05 & 0.075 & 0.10 & 0.15 & 0.20 & 0.25 & 0.50 & 0.75 & 1.00 & 1.50 & 2.00 \\
\hline \multirow{12}{*}{$0.05,1.7945$} & \multirow{3}{*}{0.30} & ARL & 372.32 & 300.30 & 267.46 & 200.13 & 126.63 & 83.99 & 50.86 & 30.83 & 23.70 & 7.94 & 4.20 & 2.67 & 1.71 & 1.34 \\
\hline & & MDRL & 229.50 & 189.50 & 164.00 & 137.50 & 90.00 & 63.00 & 43.00 & 25.00 & & 6.00 & 3.00 & 2.00 & 1.00 & 1.00 \\
\hline & & SDRL & 440.89 & 337.09 & 303.15 & 217.65 & 133.33 & 86.73 & 43.23 & 26.88 & 20.06 & 6.38 & 3.41 & 1.97 & 1.04 & 0.63 \\
\hline & \multirow{3}{*}{0.50} & ARL & 373.06 & 295.49 & 275.52 & 199.90 & 112.13 & 79.72 & 44.22 & 29.88 & 20.36 & 6.82 & 3.69 & 2.37 & 1.48 & 1.17 \\
\hline & & MDRL & 252.00 & 180.00 & 175.00 & 134.50 & 77.00 & 62.00 & 37.00 & 24.00 & 17.00 & 5.00 & 3.00 & 1.00 & 1.00 & 1.00 \\
\hline & & SDRL & 430.16 & 347.46 & 322.23 & 208.37 & 123.57 & 80.04 & 40.82 & 25.98 & 17.17 & 5.98 & 3.05 & 1.92 & 0.96 & 0.51 \\
\hline & \multirow{3}{*}{0.70} & ARL & 372.74 & 292.94 & 265.49 & 165.08 & 103.54 & 73.36 & 39.55 & 25.23 & 18.63 & 6.20 & 3.22 & 2.12 & 1.35 & 1.15 \\
\hline & & MDRL & 232.50 & 193.00 & 175.00 & 104.50 & 71.50 & 57.00 & 31.00 & 20.00 & 15.00 & 5.00 & 2.00 & 2.00 & 1.00 & 1.00 \\
\hline & & SDRL & 445.33 & & 295.51 & 187.21 & 111.53 & 69.68 & 36.46 & 21.31 & & 5.14 & 2.63 & 1.53 & 0.72 & 0.44 \\
\hline & \multirow{3}{*}{0.90} & ARL & 373.19 & 260.06 & 233.04 & 141.73 & & 52.56 & 30.20 & 20.80 & & 4.66 & 2.49 & 1.72 & 1.21 & 1.07 \\
\hline & & MDRL & 250.50 & 175.00 & 152.00 & 92.00 & 63.00 & 41.00 & 25.50 & 16.50 & 13.00 & 3.00 & 2.00 & 1.00 & 1.00 & 1.00 \\
\hline & & SDRL & 504.40 & 293 & & 154 & & & & & & 4.00 & 1.84 & 1.11 & 56 & 0.30 \\
\hline \multirow{12}{*}{$0.10,2.0705$} & \multirow{3}{*}{0.30} & $\mathrm{~L}$ & 3 & 5 & 9 & 5 & 3 & 84 & 57 & 2 & 2 & 9.23 & 5.17 & 37 & 44 & 2.14 \\
\hline & & MDRL & 258.50 & & 20 & 0 & 10 & & 44.00 & & & 7.00 & 4.00 & 3.00 & 2.00 & 2.00 \\
\hline & & SDRL & 393.33 & & 292 & 24 & 14 & & 53.04 & & & 6.67 & 3.47 & 1.91 & 0.91 & 0.48 \\
\hline & \multirow{4}{*}{0.50} & ARL & 372.70 & 310.23 & 288.24 & 218.26 & 136.05 & & 49.92 & 32.06 & & 8.14 & 4.41 & 2.92 & 1.75 & 1.37 \\
\hline & & MDRL & 258.00 & 194.50 & 199.00 & 145.00 & 93.00 & 69.00 & 41.00 & 28.00 & 20.00 & 7.00 & 4.00 & 2.00 & 1.00 & 1.00 \\
\hline & & SDRL & 387.69 & 346.88 & 301.66 & 227.18 & 141.27 & 92.76 & 44.89 & 25.06 & 17.63 & 6.16 & 3.29 & 2.08 & 1.12 & 0.70 \\
\hline & & ARL & 372.71 & 295.91 & 285.55 & 191.25 & 123.55 & 80.58 & 46.97 & 28.28 & 19.62 & 7.11 & 3.81 & 2.55 & 1.62 & 1.35 \\
\hline & \multirow[t]{2}{*}{0.70} & MDRL & 235.00 & 203.50 & 188.00 & 130.00 & 84.50 & 59.00 & 38.00 & 24.00 & 18.00 & 6.00 & 3.00 & 2.00 & 1.00 & 1.00 \\
\hline & & SDRL & 406.83 & 305.18 & 308.32 & 192.77 & 126.24 & 76.18 & 39.86 & 22.44 & 14.28 & 5.12 & 2.62 & 1.64 & 0.89 & 0.61 \\
\hline & \multirow{3}{*}{0.90} & ARL & 373.16 & & 25 & 160 & & & 34 & & & 5.35 & 2.83 & 1.94 & 1.32 & 1.14 \\
\hline & & MDRL & 251.00 & 198.50 & 172.50 & 108.50 & 6 & 4 & 29.00 & 19.00 & & 5.00 & 2.00 & 1.00 & 1.00 & 1.00 \\
\hline & & SDRL & 421.97 & 306.04 & 267.54 & 167.52 & 98.15 & 58.01 & 28.40 & 16.22 & 12.17 & 3.68 & 1.96 & 1.23 & 0.65 & 0.42 \\
\hline \multirow{12}{*}{$0.25,2.464$} & \multirow{3}{*}{0.30} & & 8.53 & 6.72 & 36 & 27 & 21 & 93 & 81 & 1 & 52 & 10.03 & 5.58 & 3.98 & 2.72 & 2.33 \\
\hline & & MDRL & 261.50 & 00 & & & & & & & & 9.00 & 5.00 & 4.00 & 2.00 & 2.00 \\
\hline & & SDRL & 368.10 & & 330 & 269.50 & & & & & & 5.81 & 3.02 & 1.88 & 1.04 & 0.68 \\
\hline & \multirow{4}{*}{0.50} & & & & & 3 & & & & 9 & & 9.22 & 5.26 & 3.81 & 2.57 & 2.20 \\
\hline & & MDRL & 279.50 & 227.00 & 210.00 & 184.00 & 138.00 & 9 & 54.50 & 30.00 & 23.00 & 8.00 & 5.00 & 3.00 & 2.00 & 2.00 \\
\hline & & SDRL & 375.56 & 371.42 & 321.47 & 276.45 & 188.26 & 137.47 & 64.90 & 40.37 & 22.72 & 5.79 & 2.85 & 1.80 & 0.91 & 0.51 \\
\hline & & & 372.39 & 324.77 & 314.90 & 250.82 & 176.30 & 120.35 & 61.20 & 36.69 & & 7.91 & 4.57 & 3.29 & 2.40 & 2.16 \\
\hline & 0.70 & MDRL & 257.00 & 210.00 & 213.00 & 177.50 & 121.00 & 84.00 & 45.00 & 28.00 & 20.00 & 7.00 & & 3.00 & 2.00 & 2.00 \\
\hline & & SDRL & 364.03 & 337.33 & 327.42 & 255.45 & 176.38 & 111.55 & 56.76 & 30.39 & 19.26 & 4.53 & 2.32 & 1.46 & 0.77 & 0.46 \\
\hline & & ARL & 372.90 & 311.54 & 288.37 & 207.47 & 141.87 & & 45.25 & 25.95 & 17.77 & 5.83 & 3.25 & 2.34 & 1.59 & 1.31 \\
\hline & 90 & MDRL & 258.00 & 206.50 & 203.00 & 137.00 & 101.00 & 62.00 & 33.00 & 20.00 & 14.00 & 5.00 & 3.00 & 2.00 & 1.00 & 1.00 \\
\hline & & SDRL & 396.02 & 306.39 & 282 & 215.36 & 13 & 98.06 & 40.92 & 22.40 & 13.55 & 3.52 & 1.89 & 1.22 & 0.74 & 0.52 \\
\hline & & & & & & & & & & & & 12 & & 4 & 2.93 & 2.54 \\
\hline & 0.30 & MDRL & 257.00 & 242.00 & 257.00 & 224.00 & 175 & 146.00 & 90 & 56.50 & & 9.00 & 5.00 & 4.00 & 3.00 & 2.00 \\
\hline & & SDRL & 378.95 & 348.15 & 372.31 & 291.54 & 270.59 & 208.75 & 113.47 & 71.50 & 48.47 & 8.60 & 3.41 & 1.99 & 0.91 & 0.67 \\
\hline & & ARL & 368.91 & 357.44 & 337.55 & 291.16 & 255.87 & 186.62 & 108.46 & 70.63 & 46.00 & 10.97 & 5.52 & 3.90 & 2.64 & 2.29 \\
\hline & 0.50 & MDRL & 250.00 & 237.50 & 235.50 & 208.00 & 182.50 & & & 53.00 & 32.00 & 9.00 & 5.00 & 4.00 & 2.00 & 2.00 \\
\hline & & SDRL & 375.15 & 361.59 & 327.06 & 275.20 & 240.18 & 183.41 & 102.22 & 64.90 & 41.90 & 7.81 & 3.20 & 1.67 & 0.85 & 0.58 \\
\hline & & ARL & 367.89 & 304.77 & 311.64 & 256.76 & 217.63 & 164.01 & 91.45 & 56.02 & 37.31 & 9.03 & 4.76 & 3.41 & 2.48 & 2.19 \\
\hline & 0.70 & MDRL & 229.00 & 215.00 & 210.00 & 182.50 & 149.50 & 113.00 & 66.50 & 40.00 & 25.00 & 8.00 & 4.00 & 3.00 & 2.00 & 2.00 \\
\hline & & SDRL & 323.61 & 293.02 & 319.15 & 243.85 & 217.08 & 162.08 & 86.30 & 50.81 & 36.10 & 6.05 & 2.48 & 1.40 & 0.70 & 0.44 \\
\hline & & ARL & 371.53 & 294.00 & 283.18 & 233.03 & 175.14 & 119.40 & 64.27 & 39.42 & 24.28 & 6.18 & 3.48 & 2.45 & 1.66 & 1.31 \\
\hline & 0.90 & MDRL & 227.00 & 189.00 & 201.50 & 159.00 & 128.00 & 79.50 & 44.00 & 28.00 & 17.00 & 5.00 & 3.00 & 2.00 & 1.00 & 1.00 \\
\hline & & SDRL & 332.40 & 313.32 & 276.24 & 233.78 & 170.76 & 120.14 & 61.63 & 37.78 & 23.13 & 4.12 & 2.00 & 1.31 & 0.77 & 0.54 \\
\hline
\end{tabular}

brief, the proposed TEWMA - $\mathrm{SR}_{\mathrm{RSS}}$ control chart is quite sensitive for detecting process location shifts relative to the TEWMA - $\bar{X}$ control chart.

4.4. Proposed versus DEWMA-SR $R_{R S}$ Control Chart. The proposed TEWMA - $\mathrm{SR}_{\mathrm{RSS}}$ control chart provides better performance than the DEWMA - $\mathrm{SR}_{\mathrm{RSS}}$ control chart. For example, under a normal environment, at $n=10$ and $\lambda=$ 0.50 for $\delta=2.5$ percent, the proposed TEWMA - SR $\mathrm{RSS}$ control chart reduces the $\mathrm{ARL}_{1}$ by 44.92 percent, while the DEWMA - SR RSS control chart reduces the $\mathrm{ARL}_{1}$ by 39.99 percent (see Table 8). Similarly, when we examine the Logistic distribution at $n=5$ and $\lambda=0.25$ for $\delta=3$ percent, the

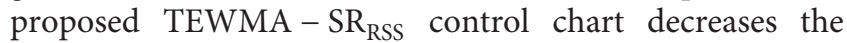
$\mathrm{ARL}_{1}$ by 34.59 percent and the DEWMA - SR RSS control 


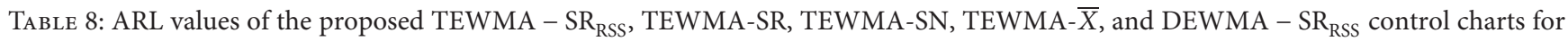
$\lambda=0.05$ under various distributions when $\mathrm{ARL}_{0}=370$..

\begin{tabular}{|c|c|c|c|c|c|c|c|c|}
\hline$\delta$ & Control charts & $L$ & Normal & $t_{4}$ & $t_{8}$ & Logistic & Laplace & $\mathrm{CN}$ \\
\hline \multicolumn{9}{|c|}{$n=5$} \\
\hline \multirow{5}{*}{0.00} & TEWMA - SR RSS & 1.585 & 371.11 & 369.03 & 370.51 & 371.69 & 371.65 & 369.92 \\
\hline & TEWMA-SR & 1.761 & 370.21 & 370.70 & 370.50 & 369.93 & 369.93 & 370.41 \\
\hline & TEWMA-SN & 1.755 & 370.12 & 371.34 & 369.35 & 370.63 & 370.63 & 371.45 \\
\hline & TEWMA $-\bar{X}$ & 1.761 & 370.42 & 384.87 & 375.61 & 369.18 & 368.30 & 420.27 \\
\hline & DEWMA - SR $R_{\text {RSS }}$ & 1.742 & 370.75 & 369.11 & 37.065 & 369.58 & 368.53 & 371.09 \\
\hline \multirow{5}{*}{0.05} & TEWMA - SR ${ }_{R S S}$ & 1.585 & 92.67 & 111.34 & 99.36 & 80.72 & 63.49 & 94.31 \\
\hline & TEWMA-SR & 1.761 & 170.21 & 133.43 & 154.45 & 152.63 & 117.81 & 164.23 \\
\hline & TEWMA-SN & 1.755 & 215.69 & 147.36 & 177.50 & 173.25 & 107.59 & 190.75 \\
\hline & TEWMA $-\bar{X}$ & 1.761 & 157.04 & 159.27 & 155.59 & 156.45 & 156.49 & 164.35 \\
\hline & DEWMA - SR ${ }_{\text {RSS }}$ & 1.742 & 95.77 & 114.90 & 101.40 & 84.81 & 66.13 & 97.51 \\
\hline \multirow{5}{*}{0.10} & TEWMA - SR RSS & 1.585 & 33.91 & 41.65 & 37.64 & 30.49 & 22.54 & 35.63 \\
\hline & TEWMA-SR & 1.761 & 71.18 & 52.78 & 63.28 & 63.83 & 47.12 & 68.51 \\
\hline & TEWMA-SN & 1.755 & 102.07 & 59.59 & 75.47 & 74.30 & 43.97 & 84.11 \\
\hline & TEWMA $-\bar{X}$ & 1.761 & 63.92 & 64.94 & 63.34 & 64.59 & 64.94 & 65.32 \\
\hline & DEWMA - SR RSS & 1.742 & 36.12 & 45.08 & 40.27 & 33.16 & 25.23 & 37.70 \\
\hline \multirow{5}{*}{0.25} & TEWMA - SR RSS & 1.585 & 7.94 & 10.43 & 8.89 & 7.20 & 5.48 & 8.35 \\
\hline & TEWMA-SR & 1.761 & 18.95 & 13.75 & 16.66 & 16.93 & 12.66 & 18.06 \\
\hline & TEWMA-SN & 1.755 & 28.46 & 15.85 & 20.09 & 20.07 & 12.44 & 22.93 \\
\hline & TEWMA $-\bar{X}$ & 1.761 & 16.57 & 16.59 & 16.24 & 16.59 & 16.59 & 16.57 \\
\hline & DEWMA - $\mathrm{SR}_{\mathrm{RSS}}$ & 1.742 & 9.03 & 12.07 & 11.74 & 9.62 & 7.32 & 10.56 \\
\hline \multirow{5}{*}{0.50} & TEWMA - SR RSS & 1.585 & 2.35 & 3.34 & 2.80 & 2.18 & 2.01 & 2.51 \\
\hline & TEWMA-SR & 1.761 & 6.16 & 4.50 & 5.48 & 5.45 & 4.46 & 5.83 \\
\hline & TEWMA-SN & 1.755 & 9.62 & 5.20 & 6.70 & 6.62 & 4.71 & 7.60 \\
\hline & TEWMA $-\bar{X}$ & 1.761 & 5.07 & 4.99 & 5.02 & 5.10 & 5.07 & 5.00 \\
\hline & DEWMA - SR RSS & 1.742 & 2.88 & 3.91 & 2.99 & 2.78 & 2.09 & 2.84 \\
\hline \multirow{5}{*}{0.75} & TEWMA - SR RSS & 1.585 & 1.37 & 1.79 & 1.58 & 1.31 & 1.32 & 1.46 \\
\hline & TEWMA-SR & 1.761 & 3.15 & 2.49 & 2.86 & 2.88 & 2.57 & 2.98 \\
\hline & TEWMA-SN & 1.755 & 4.88 & 2.81 & 3.47 & 3.45 & 2.80 & 3.83 \\
\hline & TEWMA $-\bar{X}$ & 1.761 & 2.44 & 2.38 & 2.42 & 2.44 & 2.42 & 2.40 \\
\hline & DEWMA - SR $R_{\text {RSS }}$ & 1.742 & 1.85 & 1.99 & 1.89 & 1.73 & 1.79 & 1.72 \\
\hline \multirow{5}{*}{1.00} & TEWMA - SR RSS & 1.585 & 1.08 & 1.36 & 1.20 & 1.10 & 1.12 & 1.14 \\
\hline & TEWMA-SR & 1.761 & 2.06 & 1.77 & 1.94 & 1.95 & 1.85 & 1.97 \\
\hline & TEWMA-SN & 1.755 & 3.02 & 1.93 & 2.25 & 2.26 & 2.01 & 2.41 \\
\hline & TEWMA $-\bar{X}$ & 1.761 & 1.54 & 1.50 & 1.53 & 1.53 & 1.52 & 1.52 \\
\hline & DEWMA - SR RSS & 1.742 & 1.44 & 1.67 & 1.46 & 1.33 & 1.32 & 1.31 \\
\hline \multirow{5}{*}{1.25} & TEWMA - SR RSS & 1.585 & 1.01 & 1.09 & 1.03 & 1.01 & 1.03 & 1.01 \\
\hline & TEWMA-SR & 1.761 & 1.58 & 1.44 & 1.53 & 1.54 & 1.51 & 1.52 \\
\hline & TEWMA-SN & 1.755 & 2.14 & 1.52 & 1.69 & 1.70 & 1.61 & 1.74 \\
\hline & TEWMA $-\bar{X}$ & 1.761 & 1.19 & 1.18 & 1.19 & 1.19 & 1.19 & 1.18 \\
\hline & DEWMA - SR RSS & 1.742 & 1.03 & 1.19 & 1.09 & 1.04 & 1.07 & 1.05 \\
\hline \multirow{5}{*}{1.50} & TEWMA - SR RSS & 1.585 & 1.00 & 1.02 & 1.01 & 1.00 & 1.00 & 1.00 \\
\hline & TEWMA-SR & 1.761 & 1.33 & 1.28 & 1.31 & 1.32 & 1.33 & 1.30 \\
\hline & TEWMA-SN & 1.755 & 1.66 & 1.32 & 1.39 & 1.40 & 1.38 & 1.38 \\
\hline & TEWMA $-\bar{X}$ & 1.761 & 1.06 & 1.06 & 1.07 & 1.06 & 1.06 & 1.05 \\
\hline & DEWMA - SR RSS & 1.742 & 1.00 & 1.06 & 1.02 & 1.00 & 1.00 & 1.00 \\
\hline \multicolumn{9}{|c|}{$n=10$} \\
\hline \multirow{5}{*}{0.00} & TEWMA - SR RSS & 1.3635 & 369.61 & 370.73 & 369.67 & 371.04 & 371.04 & 369.54 \\
\hline & TEWMA-SR & 1.773 & 370.71 & 370.32 & 371.50 & 371.75 & 371.75 & 370.57 \\
\hline & TEWMA-SN & 1.774 & 370.63 & 369.08 & 368.98 & 370.48 & 370.48 & 371.49 \\
\hline & TEWMA $-\bar{X}$ & 1.761 & 370.31 & 378.00 & 365.57 & 366.34 & 368.59 & 425.74 \\
\hline & DEWMA - $\mathrm{SR}_{\mathrm{RSS}}$ & 1.503 & 369.55 & 369.84 & 371.06 & 371.38 & 370.20 & 370.52 \\
\hline \multirow{5}{*}{0.05} & TEWMA - SR ${ }_{\mathrm{RSS}}$ & 1.3635 & 34.99 & 43.49 & 40.15 & 30.63 & 23.68 & 37.52 \\
\hline & TEWMA-SR & 1.773 & 111.13 & 85.42 & 102.17 & 101.11 & 77.70 & 106.18 \\
\hline & TEWMA-SN & 1.774 & 135.28 & 94.83 & 118.69 & 115.90 & 66.23 & 129.65 \\
\hline & TEWMA $-\bar{X}$ & 1.761 & 102.68 & 105.01 & 103.54 & 102.70 & 102.90 & 105.26 \\
\hline & DEWMA - SR & 1.503 & 37.77 & 47.80 & 43.22 & 33.16 & 26.02 & 39.68 \\
\hline
\end{tabular}


TABLE 8: Continued.

\begin{tabular}{|c|c|c|c|c|c|c|c|c|}
\hline$\delta$ & Control charts & $L$ & Normal & $t_{4}$ & $t_{8}$ & Logistic & Laplace & $\mathrm{CN}$ \\
\hline \multirow{5}{*}{0.10} & TEWMA - SR & 1.3635 & 12.62 & 15.90 & 14.78 & 10.77 & 7.75 & 13.22 \\
\hline & TEWMA-SR & 1.773 & 42.69 & 31.60 & 38.05 & 38.30 & 29.06 & 40.28 \\
\hline & TEWMA-SN & 1.774 & 53.12 & 35.75 & 45.53 & 44.56 & 25.67 & 50.43 \\
\hline & TEWMA $-\bar{X}$ & 1.761 & 39.00 & 39.58 & 39.03 & 39.29 & 39.27 & 39.33 \\
\hline & DEWMA - SR RSS & 1.503 & 14.58 & 17.68 & 15.62 & 12.68 & 9.50 & 15.23 \\
\hline \multirow{5}{*}{0.25} & TEWMA - SR $\mathrm{RSS}$ & 1.3635 & 2.47 & 3.29 & 2.87 & 2.19 & 1.74 & 2.65 \\
\hline & TEWMA-SR & 1.773 & 10.40 & 7.46 & 9.29 & 9.26 & 7.09 & 9.84 \\
\hline & TEWMA-SN & 1.774 & 13.53 & 8.61 & 11.39 & 11.15 & 6.65 & 12.78 \\
\hline & TEWMA $-\bar{X}$ & 1.761 & 9.34 & 9.34 & 9.45 & 9.38 & 9.38 & 9.31 \\
\hline & DEWMA - SR RSS & 1.503 & 3.54 & 4.17 & 3.71 & 2.82 & 1.98 & 2.99 \\
\hline \multirow{5}{*}{0.50} & TEWMA - SR $\mathrm{RSS}$ & 1.3635 & 1.06 & 1.23 & 1.11 & 1.26 & 1.04 & 1.11 \\
\hline & TEWMA-SR & 1.773 & 3.12 & 2.35 & 2.82 & 2.78 & 2.35 & 2.95 \\
\hline & TEWMA-SN & 1.774 & 4.13 & 2.60 & 3.43 & 3.36 & 2.34 & 3.89 \\
\hline & TEWMA $-\bar{X}$ & 1.761 & 2.72 & 2.69 & 2.72 & 2.69 & 2.69 & 2.67 \\
\hline & DEWMA - SR RSS & 1.503 & 1.33 & 1.38 & 1.27 & 1.46 & 1.09 & 1.18 \\
\hline \multirow{5}{*}{0.75} & TEWMA - SR RSS & 1.3635 & 1.00 & 1.02 & 1.01 & 1.00 & 1.00 & 1.01 \\
\hline & TEWMA-SR & 1.773 & 1.62 & 1.39 & 1.53 & 1.52 & 1.43 & 1.55 \\
\hline & TEWMA-SN & 1.774 & 2.02 & 1.45 & 1.75 & 1.73 & 1.44 & 1.92 \\
\hline & TEWMA $-\bar{X}$ & 1.761 & 1.43 & 1.41 & 1.43 & 1.42 & 1.41 & 1.41 \\
\hline & DEWMA - SR RSS & 1.503 & 1.00 & 1.05 & 1.04 & 1.01 & 1.00 & 1.01 \\
\hline \multirow{5}{*}{1.00} & TEWMA - SR RSS & 1.3635 & 1.00 & 1.00 & 1.00 & 1.00 & 1.00 & 1.00 \\
\hline & TEWMA-SR & 1.773 & 1.18 & 1.12 & 1.16 & 1.16 & 1.15 & 1.15 \\
\hline & TEWMA-SN & 1.774 & 1.34 & 1.14 & 1.24 & 1.24 & 1.16 & 1.29 \\
\hline & TEWMA $-\bar{X}$ & 1.761 & 1.10 & 1.09 & 1.10 & 1.09 & 1.09 & 1.09 \\
\hline & DEWMA - SR RSS & 1.503 & 1.01 & 1.03 & 1.02 & 1.02 & 1.01 & 1.00 \\
\hline \multirow{5}{*}{1.25} & TEWMA - SR RSS & 1.3635 & 1.00 & 1.00 & 1.00 & 1.00 & 1.00 & 1.00 \\
\hline & TEWMA-SR & 1.773 & 1.04 & 1.04 & 1.05 & 1.04 & 1.05 & 1.03 \\
\hline & TEWMA-SN & 1.774 & 1.11 & 1.04 & 1.08 & 1.08 & 1.06 & 1.09 \\
\hline & TEWMA $-\bar{X}$ & 1.761 & 1.01 & 1.02 & 1.02 & 1.02 & 1.02 & 1.01 \\
\hline & DEWMA - SR RSS & 1.503 & 1.00 & 1.01 & 1.00 & 1.00 & 1.01 & 1.00 \\
\hline \multirow{5}{*}{1.50} & TEWMA - SR RSS & 1.3635 & 1.00 & 1.00 & 1.00 & 1.00 & 1.00 & 1.00 \\
\hline & TEWMA-SR & 1.773 & 1.01 & 1.01 & 1.01 & 1.01 & 1.02 & 1.01 \\
\hline & TEWMA-SN & 1.774 & 1.03 & 1.01 & 1.02 & 1.02 & 1.02 & 1.02 \\
\hline & TEWMA $-\bar{X}$ & 1.761 & 1.00 & 1.00 & 1.00 & 1.00 & 1.00 & 1.00 \\
\hline & DEWMA $-S_{\text {RSS }}$ & 1.503 & 1.00 & 1.00 & 1.00 & 1.00 & 1.00 & 1.00 \\
\hline
\end{tabular}

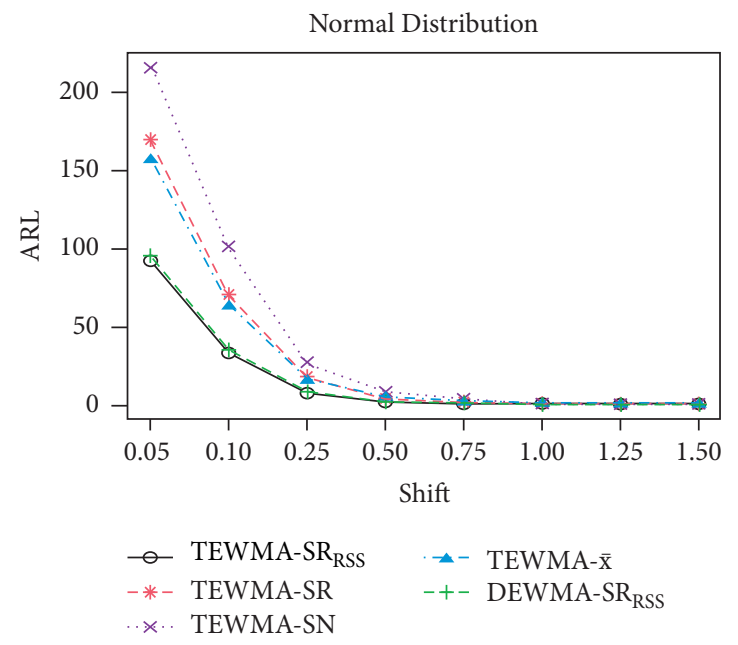

FIGURE 5: $\mathrm{ARL}_{s}$ of the proposed TEWMA - $\mathrm{SR}_{\mathrm{RSS}}$ versus TEWMA-SR, TEWMA-SN, TEWMA $-\bar{X}$, and DEWMA - SR $\mathrm{RSS}$ control charts for normal distribution when $n=5, \lambda=0.05$, and $\mathrm{ARL}_{0}=370$. 


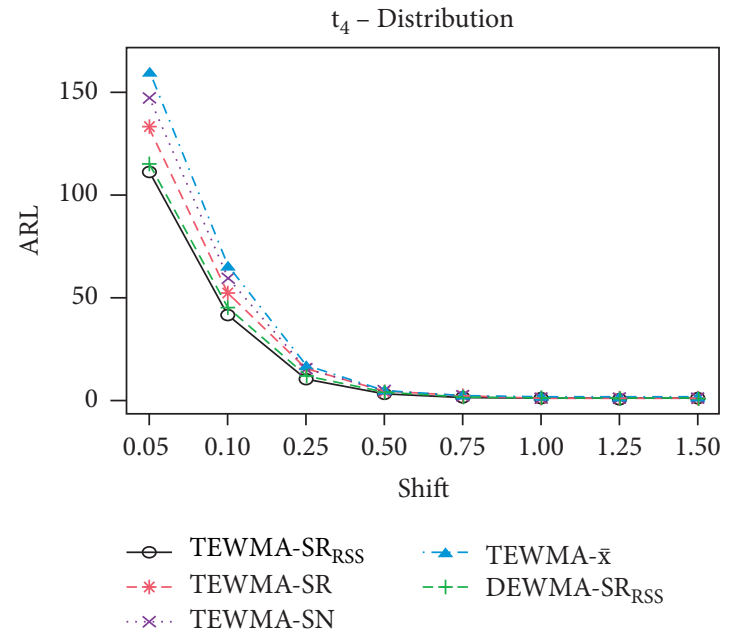

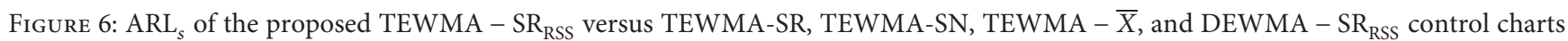
under $t_{4}$ distribution when $n=5, \lambda=0.05$, and $\mathrm{ARL}_{0}=370$.

TABLE 9: RL characteristics of the TEWMA-SR control chart under different distribution when $\mathrm{ARL}_{0}=370$.

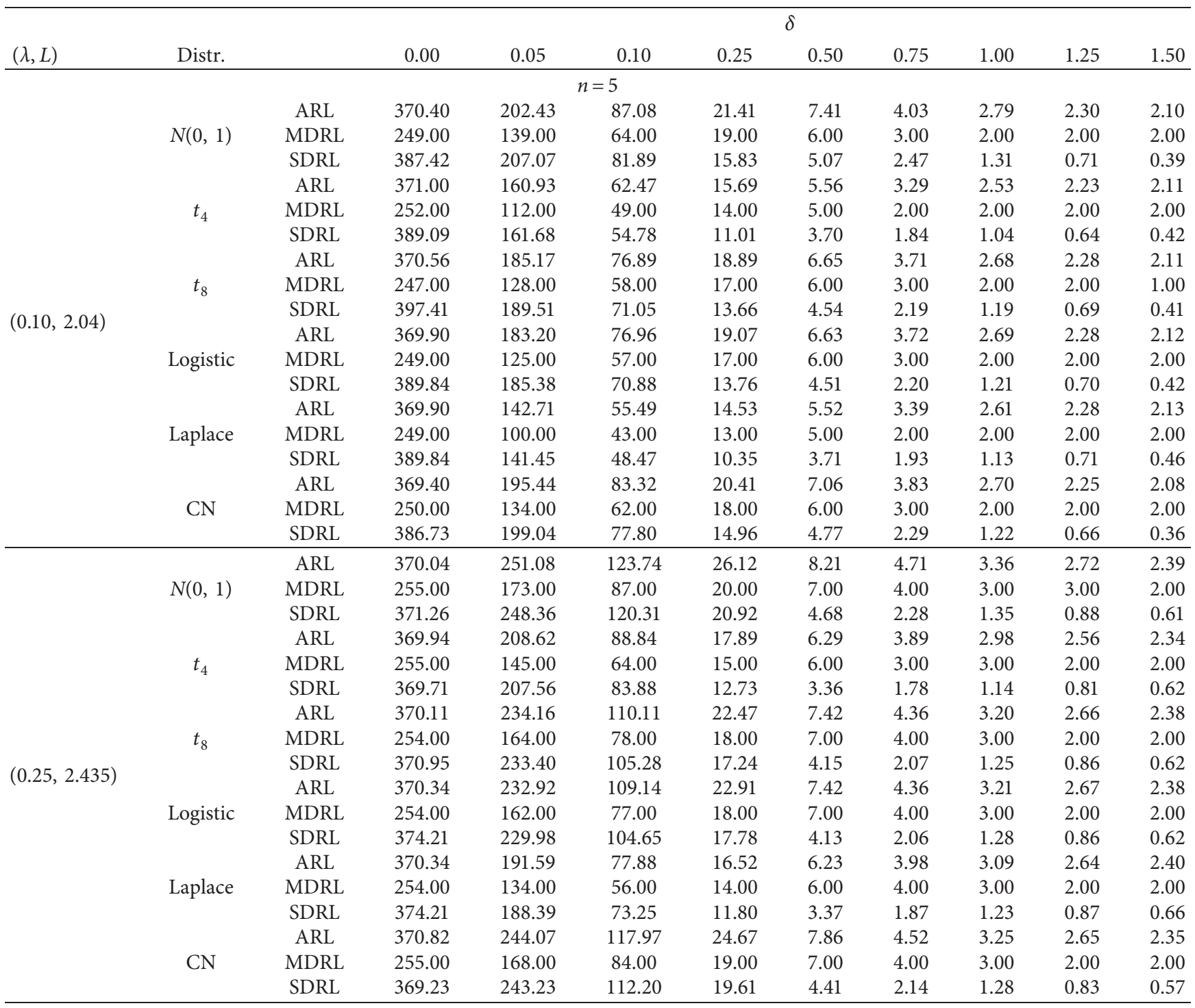


TABle 9: Continued.

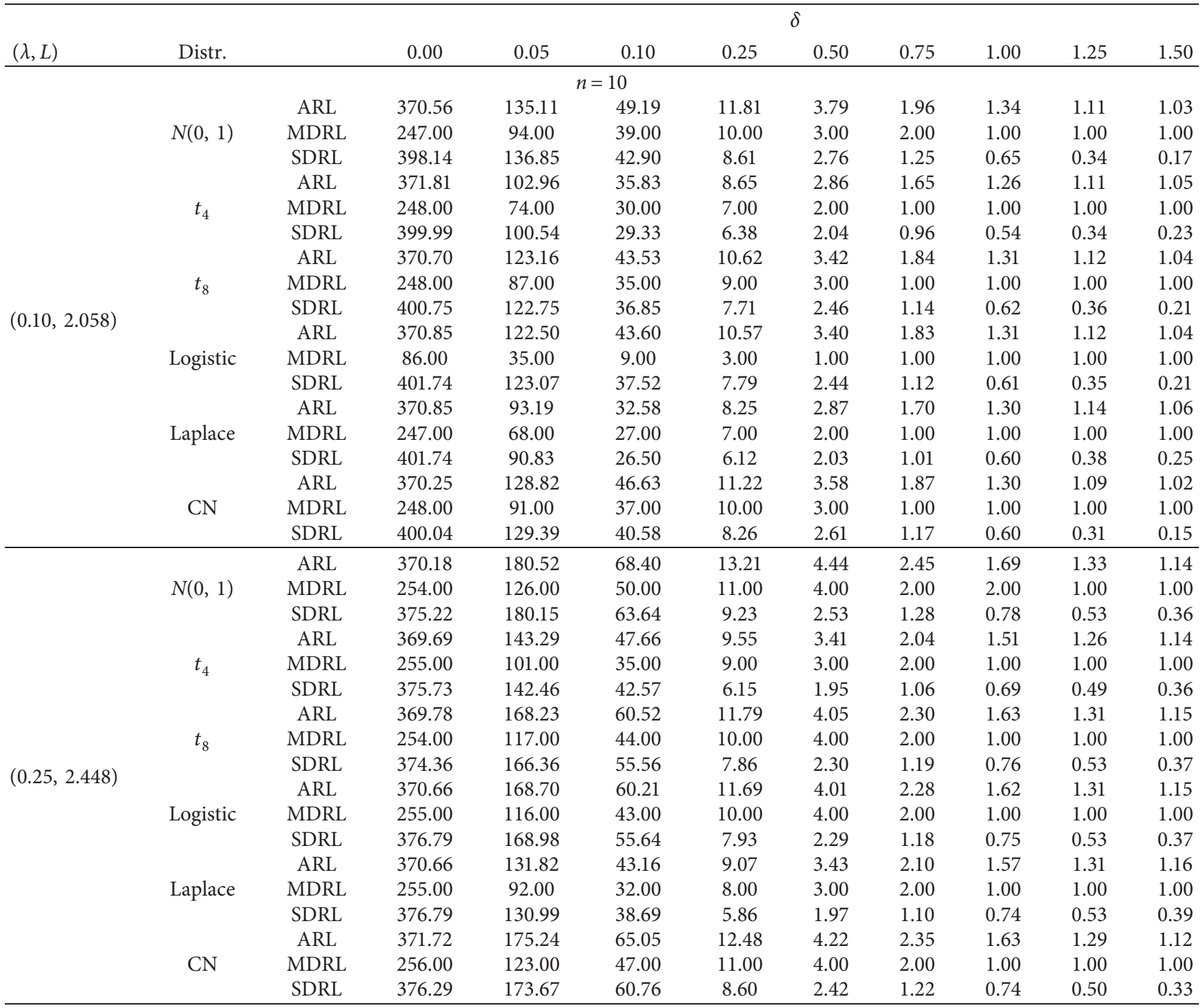

TABLE 10: RL characteristics of the TEWMA-SN control chart when $\mathrm{ARL}_{0}=370$.

\begin{tabular}{|c|c|c|c|c|c|c|c|c|c|c|c|}
\hline \multirow[b]{2}{*}{$\lambda$} & \multirow[b]{2}{*}{$L$} & & \multicolumn{9}{|c|}{$\delta$} \\
\hline & & & 0.00 & 0.05 & 0.10 & 0.25 & 0.50 & 0.75 & 1.00 & 1.25 & 1.50 \\
\hline \multicolumn{12}{|c|}{$n=5$} \\
\hline \multirow{4}{*}{0.05} & & ARL & 370.16 & 300.16 & 127.36 & 65.39 & 23.63 & 12.29 & 7.37 & 3.34 & 1.76 \\
\hline & 1.755 & MDRL & 226.00 & 189.00 & 90.00 & 52.00 & 19.00 & 10.00 & 6.00 & 3.00 & 1.00 \\
\hline & & SDRL & 433.64 & 343.98 & 132.82 & 61.43 & 20.07 & 10.43 & 6.22 & 2.64 & 1.15 \\
\hline & & ARL & 370.66 & 317.07 & 150.59 & 75.51 & 25.58 & 13.51 & 8.32 & 3.90 & 2.07 \\
\hline \multirow[t]{3}{*}{0.10} & 2.057 & MDRL & 244.00 & 209.00 & 104.00 & 56.00 & 22.00 & 12.00 & 7.00 & 3.00 & 1.00 \\
\hline & & SDRL & 403.07 & 343.76 & 154.96 & 71.44 & 20.33 & 10.12 & 6.20 & 2.87 & 1.43 \\
\hline & & ARL & 370.71 & 341.92 & 201.57 & 108.16 & 32.90 & 15.61 & 9.40 & 4.71 & 2.86 \\
\hline \multirow[t]{3}{*}{0.25} & 2.444 & MDRL & 255.00 & 236.00 & 140.00 & 77.00 & 25.00 & 13.00 & 8.00 & 4.00 & 2.00 \\
\hline & & SDRL & 376.22 & 344.07 & 198.79 & 103.51 & 27.87 & 11.13 & 5.81 & 2.49 & 1.16 \\
\hline & & ARL & 370.00 & 354.05 & 254.57 & 160.73 & 52.56 & 21.94 & 11.65 & 5.15 & 3.10 \\
\hline \multirow[t]{3}{*}{0.50} & 2.744 & MDRL & 256.00 & 244.00 & 176.00 & 112.00 & 37.00 & 16.00 & 9.00 & 5.00 & 3.00 \\
\hline & & SDRL & 373.11 & 356.39 & 252.52 & 158.19 & 49.29 & 18.87 & 8.65 & 2.69 & 1.10 \\
\hline & & ARL & 369.67 & 360.66 & 294.33 & 215.10 & 88.43 & 38.79 & 19.31 & 6.74 & 3.34 \\
\hline \multirow[t]{2}{*}{0.75} & 2.807 & MDRL & 260.00 & 249.00 & 205.00 & 149.00 & 63.00 & 27.00 & 14.00 & 5.00 & 3.00 \\
\hline & & SDRL & 367.82 & 367.82 & 358.70 & 291.34 & 213.85 & 86.50 & 36.72 & 17.46 & 4.80 \\
\hline
\end{tabular}


Table 10: Continued.

\begin{tabular}{|c|c|c|c|c|c|c|c|c|c|c|c|}
\hline \multirow[b]{2}{*}{$\lambda$} & \multirow[b]{2}{*}{$L$} & & \multicolumn{9}{|c|}{$\delta$} \\
\hline & & & 0.00 & 0.05 & 0.10 & 0.25 & 0.50 & 0.75 & 1.00 & 1.25 & 1.50 \\
\hline \multicolumn{12}{|c|}{$n=10$} \\
\hline \multirow{4}{*}{0.05} & & ARL & 370.65 & 254.20 & 80.02 & 38.63 & 13.34 & 6.56 & 3.77 & 1.68 & 1.09 \\
\hline & 1.774 & MDRL & 224.00 & 157.00 & 61.00 & 31.00 & 10.00 & 5.00 & 3.00 & 1.00 & 1.00 \\
\hline & & SDRL & 441.63 & 297.47 & 80.63 & 35.43 & 11.91 & 5.93 & 3.37 & 1.24 & 0.36 \\
\hline & & ARL & 370.66 & 280.36 & 97.51 & 45.03 & 15.25 & 7.84 & 4.72 & 2.19 & 1.30 \\
\hline \multirow[t]{3}{*}{0.10} & 2.047 & MDRL & 247.00 & 189.00 & 72.00 & 36.00 & 13.00 & 7.00 & 4.00 & 2.00 & 1.00 \\
\hline & & SDRL & 395.10 & 293.48 & 93.13 & 38.35 & 11.10 & 5.68 & 3.38 & 1.37 & 0.55 \\
\hline & & ARL & 370.39 & 316.88 & 137.44 & 61.44 & 17.26 & 8.43 & 5.16 & 2.44 & 1.35 \\
\hline \multirow[t]{3}{*}{0.25} & 2.454 & MDRL & 252.00 & 219.00 & 96.00 & 44.00 & 14.00 & 8.00 & 5.00 & 2.00 & 1.00 \\
\hline & & SDRL & 379.72 & 320.57 & 135.97 & 57.89 & 13.23 & 5.50 & 3.25 & 1.52 & 0.66 \\
\hline & & ARL & 370.52 & 337.38 & 189.41 & 97.16 & 24.83 & 10.23 & 5.77 & 2.84 & 1.77 \\
\hline \multirow[t]{3}{*}{0.50} & 2.761 & MDRL & 254.00 & 233.00 & 133.00 & 69.00 & 18.00 & 8.00 & 5.00 & 3.00 & 2.00 \\
\hline & & SDRL & 375.53 & 337.71 & 186.49 & 94.36 & 22.07 & 7.67 & 3.56 & 1.30 & 0.66 \\
\hline & & ARL & 370.35 & 351.34 & 244.79 & 148.51 & 42.51 & 15.95 & 7.76 & 3.11 & 1.79 \\
\hline \multirow[t]{2}{*}{0.75} & 2.891 & MDRL & 255.00 & 243.00 & 169.00 & 103.00 & 30.00 & 12.00 & 6.00 & 3.00 & 2.00 \\
\hline & & SDRL & 369.68 & 351.31 & 243.05 & 148.06 & 40.84 & 14.16 & 6.06 & 1.70 & 0.70 \\
\hline
\end{tabular}

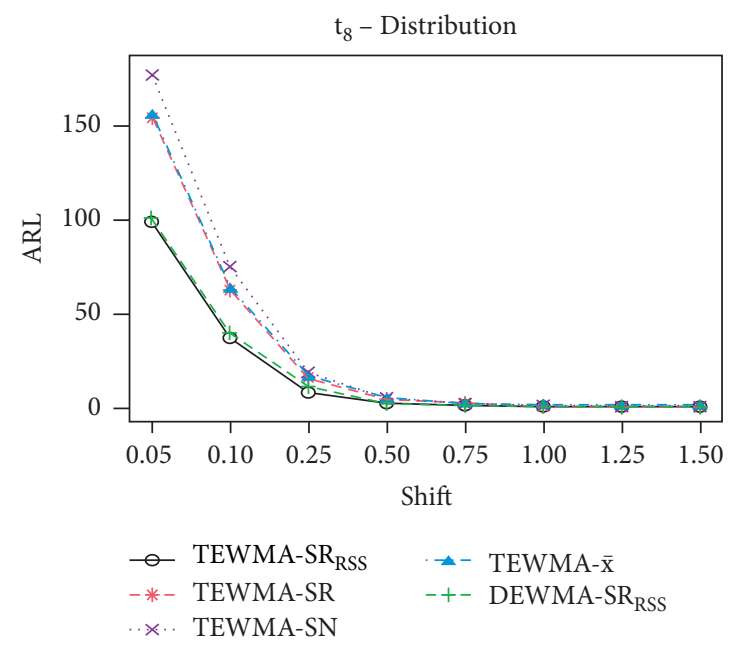

FIGURE 7: $\mathrm{ARL}_{s}$ of the proposed TEWMA - $\mathrm{SR}_{\mathrm{RSS}}$ versus TEWMA-SR, TEWMA-SN, TEWMA - $\bar{X}$, and DEWMA - SR $\mathrm{RSS}$ control charts under $t_{8}$ distribution when $n=5, \lambda=0.05$, and $\mathrm{ARL}_{0}=370$.

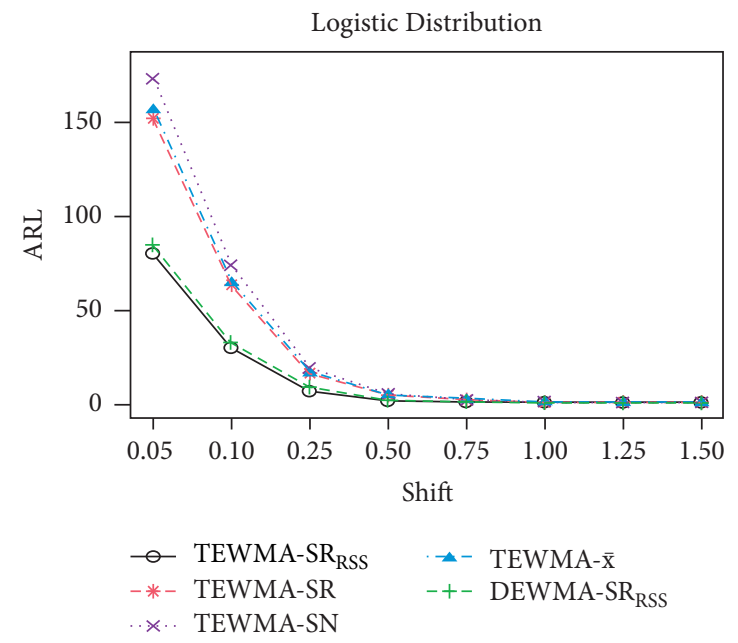

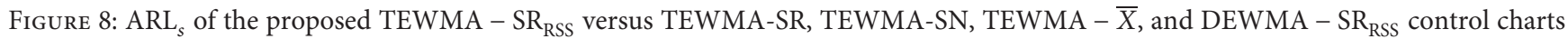
under logistic distribution when $n=5, \lambda=0.05$, and $\mathrm{ARL}_{0}=370$. 
TABLE 11: RL characteristics of the TEWMA- $\bar{X}$ control chart under different distributions when $\mathrm{ARL}_{0}=370$.

\begin{tabular}{|c|c|c|c|c|c|c|c|c|c|c|c|}
\hline \multirow[b]{2}{*}{$(\lambda, L)$} & & \multicolumn{10}{|c|}{$\delta$} \\
\hline & & & 0.00 & 0.05 & 0.10 & 0.25 & 0.50 & 0.75 & 1.00 & 1.25 & 1.50 \\
\hline \multirow{19}{*}{$(0.10,2.052)$} & \multirow{5}{*}{$N(0,1)$} & & & & $=5$ & & & & & & \\
\hline & & ARL & 369.03 & 307.06 & 200.72 & 65.87 & 21.83 & 11.54 & 7.17 & 4.79 & 3.55 \\
\hline & & MDRL & 250.00 & 202.00 & 136.00 & 49.00 & 19.00 & 10.00 & 6.00 & 4.00 & 3.00 \\
\hline & & SDRL & 395.95 & 328.82 & 211.07 & 60.94 & 16.99 & 8.57 & 5.37 & 3.60 & 2.72 \\
\hline & & ARL & 370.29 & 186.99 & 77.45 & 18.30 & 5.80 & 2.86 & 1.75 & 1.26 & 1.10 \\
\hline & \multirow[t]{2}{*}{$t_{4}$} & MDRL & 242.00 & 124.00 & 56.00 & 16.00 & 5.00 & 2.00 & 1.00 & 1.00 & 1.00 \\
\hline & & SDRL & 400.55 & 194.09 & 74.04 & 13.24 & 4.36 & 2.15 & 1.18 & 0.60 & 0.35 \\
\hline & \multirow{3}{*}{$t_{8}$} & $\mathrm{ARL}$ & 369.13 & 187.84 & 75.73 & 18.58 & 6.07 & 2.91 & 1.78 & 1.31 & 1.10 \\
\hline & & MDRL & 241.50 & 129.50 & 56.00 & 17.00 & 5.00 & 2.00 & 1.00 & 1.00 & 1.00 \\
\hline & & SDRL & 401.65 & 197.86 & 71.48 & 13.83 & 4.56 & 2.18 & 1.16 & 0.65 & 0.34 \\
\hline & \multirow{4}{*}{ Logistic } & ARL & 369.25 & 182.82 & 76.01 & 18.40 & 5.95 & 2.94 & 1.80 & 1.28 & 1.10 \\
\hline & & MDRL & 251.00 & 129.00 & 57.00 & 16.00 & 5.00 & 2.00 & 1.00 & 1.00 & 1.00 \\
\hline & & SDRL & 395.31 & 190.55 & 71.15 & 14.08 & 4.51 & 2.17 & 1.20 & 0.63 & 0.35 \\
\hline & & ARL & 369.16 & 181.96 & 76.63 & 18.55 & 5.95 & 2.85 & 1.78 & 1.31 & 1.10 \\
\hline & \multirow[t]{2}{*}{ Laplace } & MDRL & 253.00 & 130.00 & 57.00 & 16.00 & 5.00 & 2.00 & 1.00 & 1.00 & 1.00 \\
\hline & & SDRL & 396.30 & 191.07 & 72.13 & 14.25 & 4.47 & 2.07 & 1.17 & 0.64 & 0.33 \\
\hline & \multirow{3}{*}{$\mathrm{CN}$} & ARL & 370.86 & 184.21 & 75.84 & 18.45 & 5.88 & 2.84 & 1.76 & 1.30 & 1.10 \\
\hline & & MDRL & 238.00 & 127.50 & 57.00 & 16.00 & 5.00 & 2.00 & 1.00 & 1.00 & 1.00 \\
\hline & & SDRL & 406.48 & 187.56 & 70.40 & 13.78 & 4.48 & 2.11 & 1.14 & 0.65 & 0.35 \\
\hline \multirow{18}{*}{$(0.25,2.456)$} & \multirow{3}{*}{$N(0,1)$} & ARL & 369.58 & 337.05 & 253.31 & 92.03 & 26.45 & 12.90 & 8.04 & 5.49 & 4.12 \\
\hline & & MDRL & 255.00 & 237.00 & 180.00 & 63.00 & 21.00 & 11.00 & 7.00 & 5.00 & 4.00 \\
\hline & & SDRL & 371.62 & 344.14 & 249.89 & 90.27 & 21.86 & 9.25 & 5.23 & 3.44 & 2.57 \\
\hline & \multirow{4}{*}{$t_{4}$} & ARL & 370.01 & 244.15 & 112.68 & 22.79 & 6.56 & 3.35 & 2.09 & 1.50 & 1.20 \\
\hline & & MDRL & 259.00 & 163.00 & 80.00 & 18.00 & 6.00 & 3.00 & 2.00 & 1.00 & 1.00 \\
\hline & & SDRL & 374.25 & 244.05 & 106.57 & 18.02 & 4.02 & 2.03 & 1.24 & 0.79 & 0.47 \\
\hline & & $\mathrm{ARL}$ & 369.15 & 245.69 & 113.57 & 22.10 & 6.60 & 3.42 & 2.12 & 1.50 & 1.22 \\
\hline & \multirow[t]{2}{*}{$t_{8}$} & MDRL & 252.00 & 170.00 & 79.00 & 18.00 & 6.00 & 3.00 & 2.00 & 1.00 & 1.00 \\
\hline & & SDRL & 378.00 & 244.08 & 113.25 & 17.96 & 4.07 & 2.08 & 1.26 & 0.77 & 0.48 \\
\hline & \multirow{4}{*}{ Logistic } & ARL & 370.81 & 235.39 & 109.97 & 22.42 & 6.72 & 3.44 & 2.10 & 1.50 & 1.22 \\
\hline & & MDRL & 259.50 & 165.00 & 76.00 & 18.00 & 6.00 & 3.00 & 2.00 & 1.00 & 1.00 \\
\hline & & SDRL & 379.06 & 236.63 & 106.03 & 17.58 & 4.19 & 2.15 & 1.21 & 0.77 & 0.48 \\
\hline & & ARL & 370.35 & 238.73 & 113.35 & 22.41 & 6.52 & 3.39 & 2.11 & 1.50 & 1.21 \\
\hline & Laplace & MDRL & 259.00 & 165.00 & 79.00 & 18.00 & 6.00 & 3.00 & 2.00 & 1.00 & 1.00 \\
\hline & & SDRL & 385.77 & 241.88 & 109.29 & 17.63 & 3.99 & 2.11 & 1.24 & 0.77 & 0.49 \\
\hline & & $\mathrm{ARL}$ & 370.73 & 240.89 & 111.72 & 21.68 & 6.62 & 3.29 & 2.11 & 1.52 & 1.21 \\
\hline & $\mathrm{CN}$ & MDRL & 246.50 & 171.00 & 79.00 & 17.00 & 6.00 & 3.00 & 2.00 & 1.00 & 1.00 \\
\hline & & SDRL & 382.48 & 237.76 & 108.46 & 17.44 & 4.19 & 2.04 & 1.25 & 0.79 & 0.48 \\
\hline & & & & & $=10$ & & & & & & \\
\hline & & ARL & 371.96 & 311.91 & 204.02 & 64.05 & 21.87 & 11.43 & 6.99 & 4.93 & 3.50 \\
\hline & $N(0,1)$ & MDRL & 249.00 & 206.00 & 139.50 & 48.00 & 19.00 & 10.00 & 6.00 & 4.00 & 3.00 \\
\hline & & SDRL & 403.38 & 328.93 & 212.02 & 60.24 & 16.61 & 8.44 & 5.35 & 3.77 & 2.64 \\
\hline & & ARL & 370.15 & 128.10 & 45.77 & 10.67 & 3.17 & 1.60 & 1.15 & 1.04 & 1.01 \\
\hline & $t_{4}$ & MDRL & 246.00 & 91.00 & 37.00 & 9.00 & 3.00 & 1.00 & 1.00 & 1.00 & 1.00 \\
\hline & & SDRL & 397.13 & 125.50 & 39.69 & 7.95 & 2.35 & 0.99 & 0.44 & 0.22 & 0.11 \\
\hline & & ARL & 369.95 & 124.79 & 44.48 & 10.74 & 3.24 & 1.63 & 1.15 & 1.03 & 1.01 \\
\hline & $t_{8}$ & MDRL & 257.00 & 91.00 & 36.00 & 9.00 & 3.00 & 1.00 & 1.00 & 1.00 & 1.00 \\
\hline$\left(\begin{array}{lll}0 & 10 & 2 \\
2 & 052\end{array}\right)$ & & SDRL & 381.78 & 120.87 & 38.66 & 7.79 & 2.44 & 1.06 & 0.43 & 0.18 & 0.08 \\
\hline$(0.10,2.052)$ & & ARL & 370.40 & 123.96 & 44.63 & 10.63 & 3.20 & 1.62 & 1.16 & 1.03 & 1.00 \\
\hline & Logistic & MDRL & 242.00 & 91.00 & 37.00 & 9.00 & 2.00 & 1.00 & 1.00 & 1.00 & 1.00 \\
\hline & & SDRL & 402.56 & 121.81 & 37.00 & 7.85 & 2.42 & 1.00 & 0.46 & 0.18 & 0.07 \\
\hline & & $\mathrm{ARL}$ & 370.40 & 124.11 & 44.62 & 10.64 & 3.18 & 1.61 & 1.16 & 1.03 & 1.00 \\
\hline & Laplace & MDRL & 239.00 & 91.00 & 36.00 & 9.00 & 2.00 & 1.00 & 1.00 & 1.00 & 1.00 \\
\hline & & SDRL & 399.49 & 121.43 & 38.26 & 7.94 & 2.42 & 1.00 & 0.45 & 0.19 & 0.06 \\
\hline & & ARL & 368.84 & 123.63 & 43.39 & 10.44 & 3.18 & 1.64 & 1.16 & 1.03 & 1.00 \\
\hline & $\mathrm{CN}$ & MDRL & 240.00 & 85.50 & 35.00 & 9.00 & 3.00 & 1.00 & 1.00 & 1.00 & 1.00 \\
\hline & & SDRL & 397.07 & 123.35 & 36.98 & 7.95 & 2.32 & 1.02 & 0.44 & 0.17 & 0.06 \\
\hline
\end{tabular}


Table 11: Continued.

\begin{tabular}{|c|c|c|c|c|c|c|c|c|c|c|c|}
\hline \multirow{2}{*}{$(\lambda, L)$} & & & \multicolumn{8}{|c|}{$\delta$} & \multirow[b]{2}{*}{1.50} \\
\hline & & & 0.00 & 0.05 & 0.10 & 0.25 & 0.50 & 0.75 & 1.00 & 1.25 & \\
\hline \multirow{18}{*}{$(0.25,2.456)$} & \multirow{3}{*}{$N(0,1)$} & ARL & 369.37 & 330.89 & 250.40 & 91.13 & 26.73 & 12.70 & 7.73 & 5.47 & 4.09 \\
\hline & & MDRL & 253.00 & 226.00 & 172.00 & 64.00 & 21.00 & 11.00 & 7.00 & 5.00 & 4.00 \\
\hline & & SDRL & 371.41 & 339.08 & 252.87 & 88.32 & 22.37 & 8.83 & 5.08 & 3.37 & 2.56 \\
\hline & \multirow{3}{*}{$t_{4}$} & ARL & 370.58 & 179.71 & 64.07 & 12.03 & 3.76 & 1.91 & 1.29 & 1.07 & 1.01 \\
\hline & & MDRL & 251.00 & 122.00 & 46.00 & 10.00 & 3.00 & 2.00 & 1.00 & 1.00 & 1.00 \\
\hline & & SDRL & 376.77 & 186.95 & 58.95 & 8.47 & 2.27 & 1.07 & 0.57 & 0.27 & 0.11 \\
\hline & \multirow{3}{*}{$t_{8}$} & ARL & 370.68 & 171.99 & 63.60 & 11.62 & 3.76 & 1.93 & 1.26 & 1.08 & 1.01 \\
\hline & & MDRL & 251.00 & 123.00 & 45.50 & 10.00 & 3.00 & 2.00 & 1.00 & 1.00 & 1.00 \\
\hline & & SDRL & 378.68 & 167.45 & 59.57 & 8.06 & 2.34 & 1.10 & 0.54 & 0.28 & 0.12 \\
\hline & \multirow{4}{*}{ Logistic } & ARL & 370.68 & 178.24 & 62.88 & 11.51 & 3.71 & 1.93 & 1.28 & 1.07 & 1.01 \\
\hline & & MDRL & 244.50 & 123.00 & 46.00 & 10.00 & 3.00 & 2.00 & 1.00 & 1.00 & 1.00 \\
\hline & & SDRL & 382.00 & 179.31 & 59.26 & 7.90 & 2.34 & 1.10 & 0.56 & 0.27 & 0.10 \\
\hline & & ARL & 369.07 & 179.31 & 63.96 & 11.67 & 3.81 & 1.91 & 1.29 & 1.08 & 1.01 \\
\hline & \multirow[t]{2}{*}{ Laplace } & MDRL & 241.00 & 127.00 & 47.00 & 10.00 & 3.00 & 2.00 & 1.00 & 1.00 & 1.00 \\
\hline & & SDRL & 387.13 & 177.06 & 59.18 & 8.24 & 2.30 & 1.09 & 0.58 & 0.28 & 0.12 \\
\hline & \multirow{3}{*}{$\mathrm{CN}$} & ARL & 370.01 & 165.66 & 61.88 & 11.62 & 3.72 & 1.94 & 1.28 & 1.06 & 1.01 \\
\hline & & MDRL & 245.00 & 115.00 & 45.00 & 10.00 & 3.00 & 2.00 & 1.00 & 1.00 & 1.00 \\
\hline & & SDRL & 377.58 & 167.79 & 58.14 & 8.18 & 2.35 & 1.12 & 0.56 & 0.26 & 0.11 \\
\hline
\end{tabular}

TABLE 12: RL characteristics of the DEWMA $-\mathrm{SR}_{\mathrm{RSS}}$ control chart under different distributions with nominal $\mathrm{ARL}_{0}=370$ and $n=5$.

\begin{tabular}{|c|c|c|c|c|c|c|c|c|c|c|c|c|c|c|c|c|}
\hline \multirow[b]{2}{*}{$(\lambda, L)$} & & & \multicolumn{14}{|c|}{$\delta$} \\
\hline & & & 0.00 & 0.025 & 0.03 & 0.05 & 0.075 & 0.10 & 0.15 & 0.20 & 0.25 & 0.50 & 0.75 & 1.00 & 1.50 & 2.00 \\
\hline \multirow{18}{*}{$(0.05,1.742)$} & \multirow{3}{*}{$N(0,1)$} & ARL & 370.75 & 210.72 & 169.51 & 95.77 & 50.51 & 36.12 & 18.83 & 11.06 & 8.03 & 2.68 & 1.65 & 1.24 & 1.03 & 1.00 \\
\hline & & MDRL & 242.50 & 144.00 & 111.50 & 68.00 & 39.00 & 29.00 & 16.00 & 9.00 & 6.00 & 2.00 & 2.00 & 1.00 & 1.00 & 1.00 \\
\hline & & SDRL & 404.79 & 229.03 & 191.50 & 96.91 & 46.79 & 31.05 & 15.64 & 8.82 & 6.23 & 1.69 & 0.77 & 0.45 & 0.17 & 0.04 \\
\hline & \multirow{3}{*}{$\mathrm{CN}$} & ARL & 371.09 & 210.55 & 162.76 & 95.51 & 51.94 & 34.70 & 17.83 & 10.84 & 7.56 & 2.54 & 1.42 & 1.11 & 1.02 & 1.00 \\
\hline & & MDRL & 221.00 & 140.50 & 110.50 & 66.00 & 37.50 & 27.00 & 14.00 & 9.00 & 6.00 & 2.00 & 1.00 & 1.00 & 1.00 & 1.00 \\
\hline & & SDRL & 431.56 & 229.54 & 176.32 & 99.17 & 50.65 & 31.65 & 14.86 & 9.15 & 6.52 & 1.85 & 0.77 & 0.38 & 0.13 & 0.03 \\
\hline & \multirow{3}{*}{ Logistic } & ARL & 369.58 & 199.90 & 156.37 & 88.81 & 48.92 & 30.16 & 15.77 & 9.91 & 6.62 & 2.38 & 1.53 & 1.23 & 1.03 & 1.00 \\
\hline & & MDRL & 238.00 & 134.00 & 114.00 & 69.00 & 38.00 & 24.00 & 12.00 & 8.00 & 5.00 & 2.00 & 1.00 & 1.00 & 1.00 & 1.00 \\
\hline & & SDRL & 412.81 & 210.52 & 162.98 & 85.85 & 43.43 & 26.86 & 12.72 & 7.95 & 5.27 & 1.46 & 0.71 & 0.44 & 0.19 & 0.03 \\
\hline & \multirow{3}{*}{ Laplace } & ARL & 368.58 & 162.91 & 122.15 & 63.13 & 34.15 & 22.23 & 11.65 & 7.40 & 5.32 & 2.04 & 1.49 & 1.22 & 1.04 & 1.01 \\
\hline & & MDRL & 238.00 & 114.50 & 89.50 & 49.00 & 28.00 & 19.00 & 9.00 & 6.00 & 4.00 & 2.00 & 1.00 & 1.00 & 1.00 & 1.00 \\
\hline & & SDRL & 412.81 & 162.22 & 120.29 & 58.17 & 28.55 & 18.03 & 9.33 & 5.69 & 4.09 & 1.18 & 0.68 & 0.45 & 0.21 & 0.10 \\
\hline & \multirow{3}{*}{$t_{4}$} & ARL & 369.11 & 260.66 & 221.13 & 116.90 & 68.75 & 45.08 & 24.51 & 16.14 & 12.07 & 3.31 & 1.94 & 1.49 & 1.15 & 1.06 \\
\hline & & MDRL & 216.50 & 164.00 & 137.00 & 77.00 & 49.00 & 32.00 & 17.00 & 11.00 & 8.00 & 3.00 & 2.00 & 1.00 & 1.00 & 1.00 \\
\hline & & SDRL & 395.36 & 262.45 & 218.25 & 119.42 & 65.47 & 36.60 & 18.90 & 11.46 & 7.96 & 2.24 & 1.07 & 0.69 & 0.38 & 0.25 \\
\hline & \multirow{3}{*}{$t_{8}$} & ARL & 370.65 & 224.56 & 205.57 & 99.40 & 60.73 & 37.27 & 20.81 & 12.11 & 8.74 & 2.92 & 1.76 & 1.36 & 1.07 & 1.02 \\
\hline & & MDRL & 250.00 & 146.50 & 139.00 & 73.00 & 46.00 & 30.00 & 17.00 & 10.00 & 7.00 & 2.00 & 2.00 & 1.00 & 1.00 & 1.00 \\
\hline & & SDRL & 423.83 & 246.91 & 220.39 & 94.68 & & 31.40 & & & 6.98 & 1.98 & 0.90 & 0.60 & 0.27 & 0.15 \\
\hline \multirow{18}{*}{$(0.10,2.009)$} & \multirow{3}{*}{$N(0,1)$} & $\mathrm{L}$ & & 23 & 2 & 126 & 6 & 88 & 96 & 12 & 9 & 3.04 & 1.71 & 1.27 & 1.03 & 1.00 \\
\hline & & MDRL & 243.50 & 172.50 & 143.00 & 86 & 48.00 & 32.00 & 17.00 & 11.00 & 8.00 & 3.00 & 1.00 & 1.00 & 1.00 & 1.00 \\
\hline & & SDRL & 403.68 & 249.20 & 224.65 & 119.53 & 64.08 & 33.64 & 16.10 & 9.27 & 5.94 & 1.97 & 0.95 & 0.51 & 0.17 & 0.03 \\
\hline & \multirow{3}{*}{$\mathrm{CN}$} & ARL & 371.13 & 228.27 & 192.20 & 114.49 & 64.74 & 40.05 & 21.16 & 12.98 & 9.29 & 3.19 & 1.80 & 1.33 & 1.05 & 1.01 \\
\hline & & MDRL & 255.00 & 159.50 & 140.50 & 84.00 & 49.00 & 31.00 & 17.00 & 11.00 & 8.00 & 3.00 & 2.00 & 1.00 & 1.00 & 1.00 \\
\hline & & SDRL & 391.82 & & 186.98 & & & 34.12 & 16.85 & 9.90 & 6.45 & 2.04 & 0.96 & 0.55 & 0.23 & 0.07 \\
\hline & \multirow{3}{*}{ Logistic } & ARL & 370.86 & 227.50 & 204.89 & 106.78 & 56.34 & 35.28 & 17.38 & 11.27 & 8.29 & 2.61 & 1.64 & 1.22 & 1.03 & 1.01 \\
\hline & & MDRL & 256.00 & 159.00 & 134.50 & 77.50 & 42.00 & 27.00 & 15.00 & 10.00 & 8.00 & 2.00 & 1.00 & 1.00 & 1.00 & 1.00 \\
\hline & & SDRL & 402.88 & 233.86 & 220.06 & 106.81 & 52.13 & 29.45 & 13.21 & 8.28 & 5.45 & 1.66 & 0.87 & 0.49 & 0.17 & 0.09 \\
\hline & \multirow{4}{*}{ Laplace } & ARL & 369.36 & 189.52 & 158.15 & 79.33 & 41.15 & 25.42 & 13.29 & 8.50 & 5.99 & 2.38 & 1.51 & 1.23 & 1.04 & 1.01 \\
\hline & & MDRL & 263.00 & 135.00 & 111.50 & 60.00 & 33.00 & 21.00 & 11.00 & 7.00 & 5.00 & 2.00 & 1.00 & 1.00 & 1.00 & 1.00 \\
\hline & & SDRL & 412.96 & 194.09 & 164.28 & 74.58 & 34.35 & 22.25 & 10.10 & 5.82 & 4.17 & 1.46 & 0.76 & 0.50 & 0.21 & 0.09 \\
\hline & & ARL & 370.78 & 292.00 & 249.00 & 149.68 & 86.88 & 58.79 & 29.22 & 17.89 & 13.34 & 3.84 & 2.15 & 1.55 & 1.18 & 1.05 \\
\hline & \multirow[t]{3}{*}{$t_{4}$} & MDRL & 223.50 & 179.50 & 161.00 & 97.00 & 58.00 & 41.00 & 22.00 & 13.50 & 10.00 & 3.00 & 2.00 & 1.00 & 1.00 & 1.00 \\
\hline & & SDRL & 360.78 & 286.72 & 250.57 & 141.86 & 74.48 & 48.32 & 21.16 & 11.95 & 8.11 & 2.49 & 1.35 & 0.80 & 0.45 & 0.22 \\
\hline & & ARL & 371.53 & 256.23 & 227.76 & 132.70 & 70.13 & 46.42 & 22.92 & 14.63 & 9.97 & 3.37 & 1.90 & 1.39 & 1.08 & 1.01 \\
\hline & \multirow[t]{2}{*}{$t_{8}$} & MDRL & 256.50 & 175.50 & 156.50 & 93.00 & 51.00 & 38.00 & 20.00 & 12.50 & 9.00 & 3.00 & 2.00 & 1.00 & 1.00 & 1.00 \\
\hline & & SDRL & 390.44 & 267.12 & 235.60 & 134.53 & 66.18 & 38.63 & 17.36 & 10.85 & 7.30 & 2.12 & 1.04 & 0.67 & 0.28 & 0.11 \\
\hline
\end{tabular}


TABle 12: Continued.

\begin{tabular}{|c|c|c|c|c|c|c|c|c|c|c|c|c|c|c|c|c|}
\hline \multirow[b]{2}{*}{$(\lambda, L)$} & & & \multicolumn{14}{|c|}{$\delta$} \\
\hline & & & 0.00 & 0.025 & 0.03 & 0.05 & 0.075 & 0.10 & 0.15 & 0.20 & .25 & 0.50 & 0.75 & 1.00 & 1.50 & 0 \\
\hline \multirow{18}{*}{$(0.25,2.362)$} & \multirow{3}{*}{$N(0,1)$} & ARL & 370.58 & 01.13 & 273.03 & 167.12 & 102.33 & 58.70 & 30.54 & 17.37 & 10.93 & 3.47 & 2.04 & 1.47 & 1.08 & 1.01 \\
\hline & & MDRL & 258.50 & 199.50 & 189.00 & 110.00 & 75.00 & 43.00 & 23.00 & 14.00 & 9.00 & 3.00 & 2.00 & 1.00 & 1.00 & 1.00 \\
\hline & & SDRL & 362.34 & 306.15 & 284.31 & 169.26 & 99.93 & 54.43 & 26.51 & 13.30 & 7.40 & 1.99 & 0.99 & 0.65 & 0.27 & 0.08 \\
\hline & \multirow{3}{*}{$\mathrm{CN}$} & ARL & 371.19 & 294.66 & 257.26 & 177.09 & 104.49 & 63.79 & 29.44 & 17.46 & 11.15 & 3.60 & 2.14 & 1.51 & 1.10 & 1.02 \\
\hline & & MDRL & 274.50 & 202.00 & 176.50 & 128.00 & 72.00 & 47.00 & 21.00 & 14.00 & 9.00 & 3.00 & 2.00 & 1.00 & 1.00 & 1.00 \\
\hline & & SDRL & 353.65 & 299.44 & 256.86 & 167.69 & 105.05 & 58.35 & 26.81 & 14.12 & 8.05 & 2.05 & 1.07 & 0.67 & 0.31 & 0.15 \\
\hline & \multirow{4}{*}{ Logistic } & ARL & 371.17 & 277.17 & 257.67 & 149.26 & & 52.45 & 23.74 & & 9.30 & 3.11 & 1.91 & 1.39 & 08 & 1.02 \\
\hline & & MDRL & 275.00 & 188.00 & 176.00 & 101.50 & 60.00 & 40.00 & 18.00 & 11.00 & 8.00 & 3.00 & 2.00 & 1.00 & 1.00 & 1.00 \\
\hline & & SDRL & 348.81 & 281.41 & 258.33 & 150.34 & 81.19 & 43.81 & 20.29 & 9.88 & 6.42 & 1.75 & 0.99 & 0.62 & 0.29 & 0.14 \\
\hline & & ARL & 371.11 & 247.40 & 222.46 & 116.11 & 59.40 & 36.86 & 16.14 & 9.95 & 7.10 & 2.72 & 1.68 & 1.36 & 1.11 & 1.02 \\
\hline & \multirow[t]{3}{*}{ Laplace } & MDRL & 275.00 & 162.50 & 165.00 & 85.00 & 43.00 & 27.00 & 13.00 & 8.00 & 6.00 & 3.00 & 1.00 & 1.00 & 1.00 & 1.00 \\
\hline & & SDRL & 348.81 & 260.58 & 212.18 & 110.40 & 55 & 32.49 & 12.30 & 7.19 & 4.63 & 1.48 & 0.86 & 0.62 & 0.33 & 0.15 \\
\hline & & ARL & 369.52 & 307.12 & 297.61 & 207.84 & 132.84 & 77.75 & 37.64 & 22.42 & 14.38 & 4.45 & 2.56 & 1.79 & 1.28 & 1.10 \\
\hline & & MDRL & 243.00 & 211.00 & 208.50 & 150.00 & 89.50 & 57.00 & 29.00 & 17.00 & 12.00 & 4.00 & 2.00 & 2.00 & 1.00 & 1.00 \\
\hline & & SDRL & 376.56 & 315.27 & 279.56 & 200.11 & 139.05 & 72.20 & 31.43 & 18.34 & 10.67 & 2.66 & 1.44 & 0.89 & 0.55 & 0.34 \\
\hline & & ARL & 369.32 & 285.23 & 271.09 & 184.52 & 109.21 & 69.61 & 31.69 & 18.25 & 11.77 & 3.82 & 2.25 & 1.62 & 1.15 & 1.05 \\
\hline & & MDRL & 255.50 & 196.50 & 196.50 & 130.00 & 81.00 & 52.00 & 24.00 & 15.00 & 10.00 & 3.00 & 2.00 & 1.00 & 1.00 & 1.00 \\
\hline & & SDRL & 354.63 & 280.49 & 266.28 & 186.00 & 102.36 & 65.63 & 27.83 & 14.36 & 8.30 & 2.23 & 1.18 & 0.77 & 0.39 & 0.22 \\
\hline \multirow{18}{*}{$(0.50,2.601)$} & \multirow{3}{*}{$N(0,1)$} & ARL & .14 & 6.08 & 95 & 232.29 & 71.68 & 87 & 50.11 & 6.02 & 6.06 & 4.19 & 2.38 & 1.79 & 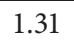 & 1.11 \\
\hline & & MDRL & 258.00 & 240.00 & 221.00 & 164.50 & 120.50 & 73.00 & 34.00 & 18.00 & 12.00 & 4.00 & 2.00 & 2.00 & 1.00 & 1.00 \\
\hline & & SDRL & 380.72 & 326.29 & 339.71 & 222.02 & 164.63 & 95.45 & 49.06 & 24.46 & 13.48 & 2.44 & 1.02 & 0.65 & 0.47 & 0.31 \\
\hline & \multirow{3}{*}{$\mathrm{CN}$} & ARL & 370.72 & 316.65 & 306.03 & 239.43 & 164.08 & 103.67 & 53.53 & 28.89 & 17.49 & 4.27 & 2.55 & 1.83 & 1.37 & 1.15 \\
\hline & & MDRL & 251.00 & 219.00 & 209.00 & 168.50 & 115.00 & 72.00 & 39.00 & 22.00 & 13.00 & 4.00 & 2.00 & 2.00 & 1.00 & 1.00 \\
\hline & & SDRL & 361.63 & 317.66 & 294.81 & 225.10 & 163.16 & 101.67 & 52.28 & 26.52 & 14.78 & 2.47 & 1.13 & 0.67 & 0.51 & 0.36 \\
\hline & \multirow{4}{*}{ Logistic } & ARL & 369.36 & 316.07 & 300.44 & 220.68 & 133.29 & 85.18 & 40.43 & 19.95 & 13.48 & 3.67 & 2.20 & 1.72 & 1.33 & 1.12 \\
\hline & & MDRL & 251.50 & 217.50 & 218.00 & 148.00 & 94.00 & 56.00 & 30.00 & 14.00 & 10.00 & 3.00 & 2.00 & 2.00 & 1.00 & 1.00 \\
\hline & & SDRL & 357.96 & 306.79 & 298.34 & 222.27 & 128.58 & 87.07 & 36.99 & 17.98 & 11.63 & 2.05 & 0.96 & 0.68 & 0.47 & 0.33 \\
\hline & & ARL & 371.17 & 291.10 & 261.49 & 170.05 & 102.20 & 61.00 & 26.56 & 13.83 & 9.45 & 3.13 & 2.05 & 1.64 & 1.31 & 1.15 \\
\hline & \multirow[t]{3}{*}{ Laplace } & MDRL & 252.50 & 208.00 & 190.50 & 120.00 & 76.00 & 42.00 & 19.00 & 10.00 & 7.00 & 3.00 & 2.00 & 2.00 & 1.00 & 1.00 \\
\hline & & SDRL & 363.24 & 282.51 & 261.00 & 168.35 & 97.94 & 57.95 & 24.30 & 11.94 & 7.29 & 1.71 & 0.94 & 0.65 & 0.48 & 0.36 \\
\hline & & ARL & 369.99 & 341.67 & 332.01 & 268.76 & 178.68 & 128.88 & 63.64 & 36.72 & 22.19 & 5.23 & 2.91 & 2.12 & 1.57 & 1.29 \\
\hline & & MDRL & 256.50 & 224.00 & 226.00 & 189.00 & 125.00 & 89.00 & 48.00 & 27.00 & 16.00 & 4.00 & 3.00 & 2.00 & 2.00 & 1.00 \\
\hline & & SDRL & 375.89 & 347.85 & 335.27 & 261.40 & 177.72 & 130.43 & 59.68 & 34.08 & 20.12 & 3.49 & 1.46 & 0.92 & 0.62 & 0.47 \\
\hline & & ARL & 370.07 & 326.33 & 319.56 & 233.66 & 169.06 & 118.75 & 56.05 & 31.40 & 19.15 & 4.50 & 2.66 & 1.95 & 1.39 & 1.19 \\
\hline & \multirow[t]{2}{*}{$t_{8}$} & MDRL & 234.50 & 223.00 & 222.00 & 164.00 & 123.00 & 88.00 & 41.00 & 22.00 & 14.00 & 4.00 & 2.00 & 2.00 & 1.00 & 1.00 \\
\hline & & SDRL & 383.44 & 327.84 & 325.64 & 228.44 & 162.83 & 113.12 & 53.16 & 29.25 & 16.76 & 2.69 & 1.24 & 0.76 & 0.51 & 0.40 \\
\hline
\end{tabular}

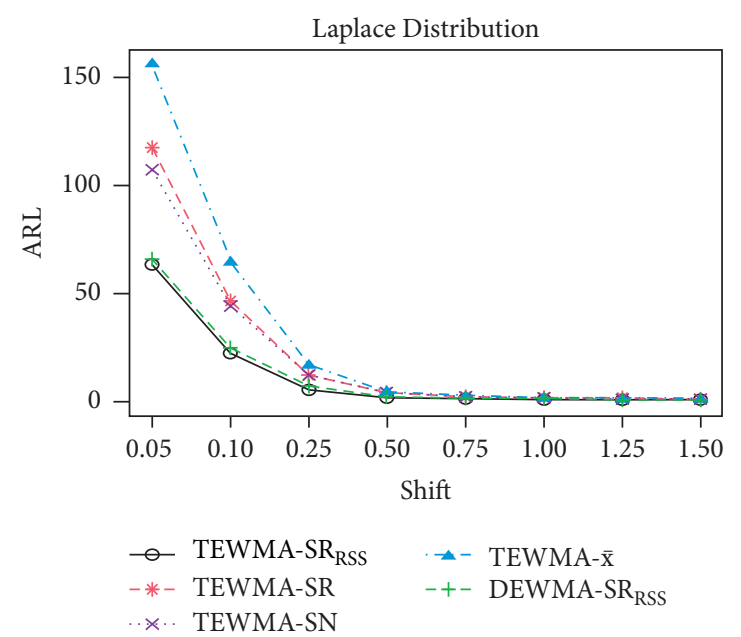

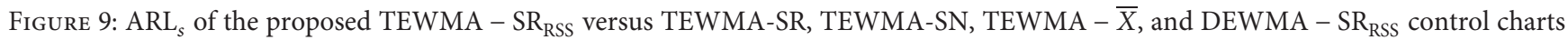
under Laplace distribution when $n=5, \lambda=0.05$, and $\mathrm{ARL}_{0}=370$. 


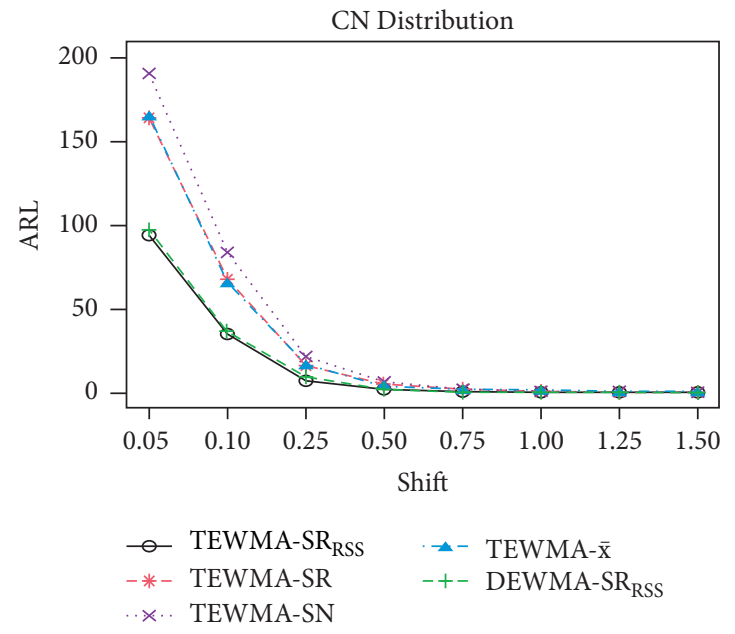

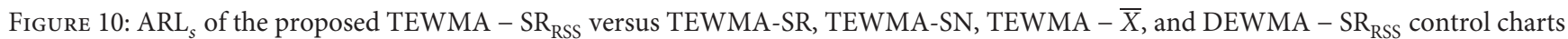
under $\mathrm{CN}$ distribution when $n=5, \lambda=0.05$, and $\mathrm{ARL}_{0}=370$.

TABLE 13: RL characteristics of the DEWMA - $\mathrm{SR}_{\mathrm{RSS}}$ control chart under different distributions with nominal $\mathrm{ARL}_{0}=370$ and $n=10$.

\begin{tabular}{|c|c|c|c|c|c|c|c|c|c|c|c|c|c|c|c|c|}
\hline \multirow[b]{2}{*}{$(\lambda, L)$} & \multirow[b]{2}{*}{ Distr. } & & \multicolumn{14}{|c|}{$\delta$} \\
\hline & & & 0.00 & 0.025 & 0.03 & 0.05 & 0.075 & 0.10 & 0.15 & 0.20 & 0.25 & 0.50 & 0.75 & 1.00 & 1.50 & 2.00 \\
\hline \multirow{18}{*}{$(0.05,1.503)$} & \multirow{3}{*}{$N(0,1)$} & ARL & 369.55 & 95.01 & 77.78 & 34.77 & 19.00 & 11.58 & 5.85 & 3.56 & 2.54 & 1.13 & 1.00 & 1.00 & 1.00 & 1.00 \\
\hline & & MDRL & 236.00 & 69.00 & 59.00 & 29.00 & 16.00 & 10.00 & 5.00 & 3.00 & 2.00 & 1.00 & 1.00 & 1.00 & 1.00 & 1.00 \\
\hline & & SDRL & 408.60 & 97.32 & 74.53 & 30.63 & 15.45 & 9.08 & 4.41 & 2.54 & 1.82 & 0.37 & 0.03 & 0.00 & 0.00 & 0.00 \\
\hline & \multirow{3}{*}{$\mathrm{CN}$} & ARL & 370.52 & 105.62 & 83.63 & 36.68 & 19.39 & 12.23 & 6.14 & 3.88 & 2.62 & 1.15 & 1.01 & 1.00 & 1.00 & 1.00 \\
\hline & & MDRL & 243.50 & 77.00 & 62.00 & 28.00 & 16.00 & 10.00 & 5.00 & 3.00 & 2.00 & 1.00 & 1.00 & 1.00 & 1.00 & 1.00 \\
\hline & & SDRL & 413.79 & 106.34 & 83.56 & 32.13 & 15.93 & 9.82 & 4.74 & 2.80 & 1.77 & 0.38 & 0.08 & 0.00 & 0.00 & 0.00 \\
\hline & \multirow{4}{*}{ Logistic } & ARL & 371.38 & 85.92 & 66.99 & 31.16 & 15.76 & 9.68 & 5.13 & 3.10 & 2.22 & 1.08 & 1.01 & 1.00 & 1.00 & 1.00 \\
\hline & & MDRL & 236.00 & 65.00 & 48.00 & 26.00 & 13.00 & 8.00 & 4.00 & 2.00 & 2.00 & 1.00 & 1.00 & 1.00 & 1.00 & 1.00 \\
\hline & & SDRL & 443.42 & 82.79 & 67.57 & 26.84 & 12.95 & 7.64 & 3.92 & 2.28 & 1.42 & 0.28 & 0.07 & 0.00 & 0.00 & 0.00 \\
\hline & & ARL & 370.20 & 68.96 & 51.92 & 24.02 & 12.46 & 7.50 & 3.87 & 2.52 & 1.85 & 1.06 & 1.00 & 1.00 & 1.00 & 1.00 \\
\hline & \multirow[t]{2}{*}{ Laplace } & MDRL & 261.00 & 52.00 & 39.00 & 20.00 & 10.00 & 6.00 & 3.00 & 2.00 & 2.00 & 1.00 & 1.00 & 1.00 & 1.00 & 1.00 \\
\hline & & SDRL & 467.24 & 65.38 & 50.61 & 19.88 & 9.92 & 5.84 & 2.87 & 1.70 & 1.10 & 0.24 & 0.06 & 0.03 & 0.00 & 0.00 \\
\hline & \multirow{4}{*}{$t_{4}$} & ARL & 369.84 & 121.67 & 100.83 & 44.80 & 22.07 & 14.68 & 7.14 & 4.70 & 3.17 & 1.28 & 1.04 & 1.00 & 1.00 & 1.00 \\
\hline & & MDRL & 263.50 & 88.00 & 72.00 & 37.00 & 18.00 & 12.00 & 6.00 & 4.00 & 2.00 & 1.00 & 1.00 & 1.00 & 1.00 & 1.00 \\
\hline & & SDRL & 427.48 & 121.96 & 99.10 & 39.37 & 18.40 & 12.18 & 5.71 & 3.49 & 2.34 & 0.54 & 0.21 & 0.05 & 0.00 & 0.00 \\
\hline & & ARL & 371.06 & 109.28 & 83.63 & 40.22 & 20.97 & 12.62 & 6.74 & 4.07 & 2.71 & 1.17 & 1.02 & 1.00 & 1.00 & 1.00 \\
\hline & \multirow[t]{2}{*}{$t_{8}$} & MDRL & 236.50 & 73.00 & 59.00 & 32.00 & 18.00 & 10.00 & 5.00 & 3.00 & 2.00 & 1.00 & 1.00 & 1.00 & 1.00 & 1.00 \\
\hline & & SDRL & 416.69 & 118.78 & 84.24 & 36.85 & 17.32 & 10.10 & 5.40 & 3.10 & 1.93 & 0.41 & 0.12 & 0.03 & 0.00 & 0.00 \\
\hline \multirow{18}{*}{$(0.10,1.709)$} & \multirow{3}{*}{$N(0,1)$} & ARL & 368.73 & 118.72 & 92.48 & 42.78 & 20.97 & 13.35 & 6.56 & 4.18 & 2.83 & 1.17 & 1.02 & 1.00 & 1.00 & 1.00 \\
\hline & & MDRL & 247.50 & 82.00 & 67.00 & 32.00 & 17.50 & 12.00 & 6.00 & 4.00 & 2.00 & 1.00 & 1.00 & 1.00 & 1.00 & 1.00 \\
\hline & & SDRL & 366.84 & 118.50 & 90.41 & 37.88 & 16.37 & 9.30 & 4.53 & 2.74 & 1.82 & 0.42 & 0.12 & 0.00 & 0.00 & 0.00 \\
\hline & \multirow{3}{*}{$\mathrm{CN}$} & ARL & 369.38 & 124.70 & 100.42 & 45.84 & 22.50 & 13.72 & 6.88 & 4.38 & 2.96 & 1.21 & 1.01 & 1.00 & 1.00 & 1.00 \\
\hline & & MDRL & 251.50 & 86.00 & 69.50 & 36.00 & 19.00 & 12.00 & 6.00 & 4.00 & 2.00 & 1.00 & 1.00 & 1.00 & 1.00 & 1.00 \\
\hline & & SDRL & 382.14 & 120.47 & 99.07 & 39.73 & 16.95 & 9.63 & 4.83 & 3.04 & 2.00 & 0.46 & 0.12 & 0.00 & 0.00 & 0.00 \\
\hline & \multirow{4}{*}{ Logistic } & ARL & 370.94 & 106.29 & 83.50 & 35.06 & 17.89 & 11.39 & 5.97 & 3.59 & 2.53 & 1.13 & 1.00 & 1.00 & 1.00 & 1.00 \\
\hline & & MDRL & 245.50 & 77.00 & 59.00 & 28.00 & 15.00 & 10.00 & 5.00 & 3.00 & 2.00 & 1.00 & 1.00 & 1.00 & 1.00 & 1.00 \\
\hline & & SDRL & 405.08 & 104.58 & 81.39 & 28.29 & 13.93 & 8.14 & 3.97 & 2.44 & 1.61 & 0.35 & 0.04 & 0.00 & 0.00 & 0.00 \\
\hline & & ARL & 369.30 & 84.66 & 61.28 & 26.85 & 13.35 & 8.47 & 4.44 & 2.87 & 2.15 & 1.10 & 1.01 & 1.00 & 1.00 & 1.00 \\
\hline & \multirow[t]{3}{*}{ Laplace } & MDRL & 257.00 & 62.00 & 46.00 & 22.00 & 11.50 & 8.00 & 4.00 & 2.00 & 2.00 & 1.00 & 1.00 & 1.00 & 1.00 & 1.00 \\
\hline & & SDRL & 448.58 & 83.63 & 57.53 & 21.01 & 9.81 & 5.86 & 3.20 & 1.82 & 1.29 & 0.33 & 0.08 & 0.00 & 0.00 & 0.00 \\
\hline & & ARL & 370.49 & 145.27 & 120.54 & 53.29 & 26.23 & 16.73 & 8.48 & 5.35 & 3.60 & 1.37 & 1.05 & 1.01 & 1.00 & 1.00 \\
\hline & \multirow[t]{3}{*}{$t_{4}$} & MDRL & 251.00 & 104.00 & 82.00 & 40.00 & 22.00 & 15.00 & 7.00 & 5.00 & 3.00 & 1.00 & 1.00 & 1.00 & 1.00 & 1.00 \\
\hline & & SDRL & 396.26 & 140.85 & 118.90 & 50.69 & 20.54 & 12.50 & 6.07 & 3.61 & 2.52 & 0.60 & 0.22 & 0.08 & 0.00 & 0.00 \\
\hline & & ARL & 371.30 & 136.63 & 106.14 & 47.20 & 23.88 & 14.28 & 7.29 & 4.68 & 3.32 & 1.25 & 1.01 & 1.00 & 1.00 & 1.00 \\
\hline & \multirow[t]{2}{*}{$t_{8}$} & MDRL & 258.00 & 93.00 & 77.00 & 36.00 & 20.00 & 12.00 & 6.00 & 4.00 & 3.00 & 1.00 & 1.00 & 1.00 & 1.00 & 1.00 \\
\hline & & SDRL & 383.52 & 136.48 & 105.01 & 42.24 & 19.12 & 11.38 & 5.14 & 3.15 & 2.15 & 0.51 & 0.10 & 0.03 & 0.00 & 0.00 \\
\hline
\end{tabular}


TABle 13: Continued.

\begin{tabular}{|c|c|c|c|c|c|c|c|c|c|c|c|c|c|c|c|c|}
\hline \multirow[b]{2}{*}{$(\lambda, L)$} & \multirow[b]{2}{*}{ Distr. } & & \multicolumn{14}{|c|}{$\delta$} \\
\hline & & & 0.00 & 0.025 & 0.03 & 0.05 & 0.075 & 0.10 & 0.15 & 0.20 & 0.25 & 0.50 & 0.75 & 1.00 & 1.50 & 2.00 \\
\hline \multirow{18}{*}{$(0.25,1.995)$} & \multirow{3}{*}{$N(0,1)$} & ARL & 368.94 & 163.89 & 125.88 & 61.02 & 28.97 & 15.79 & 7.67 & 4.75 & 3.41 & 1.36 & 1.03 & 1.00 & 1.00 & 1.00 \\
\hline & & MDRL & 261.50 & 110.00 & 86.00 & 45.00 & 20.00 & 13.00 & 7.00 & 4.00 & 3.00 & 1.00 & 1.00 & 1.00 & 1.00 & 1.00 \\
\hline & & SDRL & 361.42 & 161.56 & 121.85 & 57.69 & 26.80 & 11.94 & 5.13 & 2.78 & 1.90 & 0.57 & 0.16 & 0.00 & 0.00 & 0.00 \\
\hline & \multirow{3}{*}{$\mathrm{CN}$} & ARL & 369.14 & 179.78 & 142.14 & 60.23 & 30.03 & 16.78 & 8.48 & 5.10 & 3.62 & 1.39 & 1.05 & 1.00 & 1.00 & 1.00 \\
\hline & & MDRL & 267.00 & 121.50 & 99.50 & 44.00 & 23.00 & 13.00 & 7.00 & 5.00 & 3.00 & 1.00 & 1.00 & 1.00 & 1.00 & 1.00 \\
\hline & & SDRL & 370.83 & 176.44 & 139.84 & 55.70 & 26.50 & 13.80 & 5.81 & 3.13 & 2.13 & 0.56 & 0.21 & 0.04 & 0.00 & 0.00 \\
\hline & \multirow{3}{*}{ Logistic } & ARL & 371.19 & 151.80 & 119.49 & 52.95 & 22.92 & 13.92 & 6.63 & 4.08 & 2.94 & 1.26 & 1.02 & 1.00 & 1.00 & 1.00 \\
\hline & & MDRL & 248.00 & 105.00 & 85.00 & 37.00 & 17.50 & 11.00 & 6.00 & 4.00 & 3.00 & 1.00 & 1.00 & 1.00 & 1.00 & 1.00 \\
\hline & & SDRL & 383.41 & 152.05 & 116.71 & 51.97 & 18.61 & 10.78 & 4.24 & 2.37 & 1.60 & 0.47 & 0.14 & 0.00 & 0.00 & 0.00 \\
\hline & \multirow{3}{*}{ Laplace } & ARL & 370.19 & 121.18 & 91.21 & 36.84 & 16.40 & 9.61 & 5.06 & 3.38 & 2.52 & 1.18 & 1.02 & 1.00 & 1.00 & 1.00 \\
\hline & & MDRL & 248.00 & 88.00 & 62.00 & 27.00 & 13.00 & 8.00 & 5.00 & 3.00 & 2.00 & 1.00 & 1.00 & 1.00 & 1.00 & 1.00 \\
\hline & & SDRL & 383.41 & 116.57 & 91.76 & 30.96 & 12.75 & 6.64 & 2.99 & 1.82 & 1.27 & 0.41 & 0.13 & 0.04 & 0.00 & 0.00 \\
\hline & \multirow{3}{*}{$t_{4}$} & ARL & 369.05 & 196.86 & 156.35 & 78.67 & 36.05 & 20.40 & 9.98 & 5.87 & 4.22 & 1.60 & 1.13 & 1.02 & 1.00 & 1.00 \\
\hline & & MDRL & 247.50 & 140.00 & 108.00 & 59.00 & 26.00 & 15.00 & 9.00 & 5.00 & 4.00 & 1.00 & 1.00 & 1.00 & 1.00 & 1.00 \\
\hline & & SDRL & 376.25 & 193.47 & 162.10 & 70.86 & 30.50 & 17.24 & 6.79 & 3.74 & 2.44 & 0.72 & 0.34 & 0.15 & 0.03 & 0.00 \\
\hline & \multirow{3}{*}{$t_{8}$} & ARL & 370.83 & 197.61 & 155.40 & 76.39 & 32.26 & 19.25 & 9.26 & 5.47 & 3.89 & 1.50 & 1.06 & 1.00 & 1.00 & 1.00 \\
\hline & & MDRL & 273.00 & 134.50 & 108.00 & 55.00 & 23.00 & 15.00 & 8.00 & 5.00 & 3.00 & 1.00 & 1.00 & 1.00 & 1.00 & 1.00 \\
\hline & & SDRL & 361.65 & 203.48 & 149.05 & 73.48 & 29.81 & 15.24 & 6.87 & 3.44 & 2.16 & 0.63 & 0.24 & 0.05 & 0.00 & 0.00 \\
\hline \multirow{18}{*}{$(0.50,2.205)$} & \multirow{3}{*}{$N(0,1)$} & ARL & 370.24 & 222.16 & 196.77 & 98.15 & 47.68 & 26.30 & 10.28 & 5.80 & 3.80 & 1.47 & 1.04 & 1.00 & 1.00 & 1.00 \\
\hline & & MDRL & 261.00 & 164.00 & 138.50 & 69.00 & 33.00 & 19.00 & 8.00 & 5.00 & 3.00 & 1.00 & 1.00 & 1.00 & 1.00 & 1.00 \\
\hline & & SDRL & 362.63 & 215.71 & 194.23 & 97.34 & 47.38 & 23.66 & 8.33 & 3.79 & 2.16 & 0.60 & 0.20 & 0.03 & 0.00 & 0.00 \\
\hline & \multirow{3}{*}{$\mathrm{CN}$} & ARL & 370.38 & 228.32 & 202.24 & 102.61 & 50.84 & 28.10 & 10.92 & 6.13 & 4.10 & 1.51 & 1.08 & 1.00 & 1.00 & 1.00 \\
\hline & & MDRL & 270.00 & 159.00 & 147.50 & 72.50 & 36.00 & 20.00 & 8.00 & 5.00 & 4.00 & 1.00 & 1.00 & 1.00 & 1.00 & 1.00 \\
\hline & & SDRL & 362.46 & 225.70 & 192.80 & 98.27 & 44.75 & 25.79 & 8.48 & 4.19 & 2.37 & 0.63 & 0.26 & 0.04 & 0.00 & 0.00 \\
\hline & \multirow{4}{*}{ Logistic } & ARL & 371.47 & 226.11 & 167.01 & 89.50 & 40.36 & 22.69 & 8.90 & 4.97 & 3.40 & 1.37 & 1.05 & 1.00 & 1.00 & 1.00 \\
\hline & & MDRL & 263.50 & 147.00 & 121.00 & 67.00 & 28.00 & 16.50 & 7.00 & 4.00 & 3.00 & 1.00 & 1.00 & 1.00 & 1.00 & 1.00 \\
\hline & & SDRL & 381.73 & 255.35 & 168.83 & 83.19 & 40.27 & 20.29 & 7.19 & 3.10 & 1.83 & 0.54 & 0.22 & 0.00 & 0.00 & 0.00 \\
\hline & & ARL & 371.27 & 192.89 & 133.97 & 60.97 & 27.58 & 14.45 & 6.25 & 3.76 & 2.70 & 1.27 & 1.04 & 1.00 & 1.00 & 1.00 \\
\hline & \multirow[t]{3}{*}{ Laplace } & MDRL & 263.50 & 125.50 & 95.00 & 40.00 & 19.50 & 11.00 & 5.00 & 3.00 & 2.00 & 1.00 & 1.00 & 1.00 & 1.00 & 1.00 \\
\hline & & SDRL & 381.73 & 222.77 & 130.95 & 60.00 & 25.77 & 12.07 & 4.27 & 2.11 & 1.42 & 0.47 & 0.19 & 0.06 & 0.00 & 0.00 \\
\hline & & ARL & 370.71 & 267.22 & 213.56 & 128.25 & 64.61 & 35.50 & 14.42 & 7.45 & 5.03 & 1.75 & 1.19 & 1.04 & 1.00 & 1.00 \\
\hline & \multirow[t]{3}{*}{$t_{4}$} & MDRL & 255.00 & 186.00 & 148.50 & 92.00 & 46.00 & 25.00 & 10.00 & 6.00 & 4.00 & 2.00 & 1.00 & 1.00 & 1.00 & 1.00 \\
\hline & & SDRL & 386.36 & 261.54 & 218.84 & 123.88 & 61.63 & 33.52 & 12.57 & 5.34 & 3.18 & 0.78 & 0.39 & 0.18 & 0.03 & 0.00 \\
\hline & & ARL & 370.13 & 246.85 & 218.05 & 118.30 & 58.28 & 30.54 & 12.45 & 6.78 & 4.40 & 1.59 & 1.10 & 1.01 & 1.00 & 1.00 \\
\hline & \multirow[t]{2}{*}{$t_{8}$} & MDRL & 266.00 & 169.00 & 152.50 & 85.00 & 41.00 & 22.00 & 9.00 & 5.00 & 4.00 & 1.00 & 1.00 & 1.00 & 1.00 & 1.00 \\
\hline & & SDRL & 353.53 & 244.73 & 216.05 & 116.51 & 55.86 & 28.00 & 10.30 & 4.86 & 2.72 & 0.70 & 0.30 & 0.09 & 0.00 & 0.00 \\
\hline
\end{tabular}

chart decreases the $\mathrm{ARL}_{1}$ by 30.57 percent (see Tables 3 and 12). Furthermore, under a $\mathrm{CN}$ environment, at $n=10$ and $\lambda=0.50$ with $\delta=7.5$ percent, the proposed TEWMA - $\mathrm{SR}_{\mathrm{RSS}}$ control chart gives OOC signal after 45.28 observations, whereas the TEWMA - $\mathrm{SR}_{\mathrm{RSS}}$ control chart does after 50.84 observations (see Tables 4 and 13). The comprehensive RL features of the DEWMA - $\mathrm{SR}_{\mathrm{RSS}}$ control chart for process location shift under selected distributions are shown in Tables 12 and 13. The TEWMA - $\mathrm{SR}_{\mathrm{RSS}}$ control chart outperforms the DEWMA - $\mathrm{SR}_{\mathrm{RSS}}$ control chart, as shown in Figures 9 and 10. Results indicate that the proposed structure works effectively in all environments relative to the DEWMA - $\mathrm{SR}_{\mathrm{RSS}}$ control chart.

\section{Illustrative Example}

This section demonstrates practical application to imple-

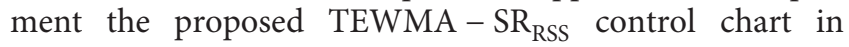
practice. For this purpose, the proposed TEWMA $-\mathrm{SR}_{\mathrm{RSS}}$ with TEWMA-SR and DEWMA - $\mathrm{SR}_{\mathrm{RSS}}$ control charts are considered to monitor the process location shift. For the practical implementation of the proposed TEWMA $-\mathrm{SR}_{\mathrm{RSS}}$ control chart, we assumed the data set used by Muttlak and Al-Sabah [20]. These data were also used by Abid et al. [37] for the execution of the EWMA - $\mathrm{SR}_{\mathrm{RSS}}$ control chart. The collected data were about the Pepsi Cola Company in ALKhobar, Saudi Arabia. The company uses different production lines, like a line used for filling soft drinks in bottles is referred to as an interest production and the volume of drink in the bottle is referred to as a variable of interest. They used the RSS method to hold the appropriate volume of soft drinks in bottles during the filling process.

The RSS technique is used to collect 27 ranked set samples by repeating the two cycles four times, with each cycle having a size of $n=3$. To compare the proposed TEWMA-SR $\mathrm{RSS}_{\text {s }}$ control chart with TEWMA-SR and DEWMA - SR RSS control charts, 27 samples with $r=12$ are collected using RSS. The design parameters $(L, \lambda)$ of the DEWMA - $\mathrm{SR}_{\mathrm{RSS}}$, TEWMA-SR, and TEWMA - $\mathrm{SR}_{\mathrm{RSS}}$ are $(1.578,0.05),(1.75,0.05)$, and $(1.305,0.05)$, respectively, at $\mathrm{ARL}_{0}=370$. Comparison of the proposed TEWMA $-\mathrm{SR}_{\mathrm{RSS}}$ control chart with the TEWMA-SR and DEWMA - SR RSS control charts can be visualized in Figures 11-13. The DEWMA - $\mathrm{SR}_{\mathrm{RSS}}$ control chart shows 4 OOC signals (at sample numbers 24-27), whereas the TEWMA-SR control chart shows no OOC signals. In contrast, the proposed TEWMA - $\mathrm{SR}_{\mathrm{RSS}}$ control chart shows 7 OOC signals (at sample numbers 21-27) in the process location parameter. 


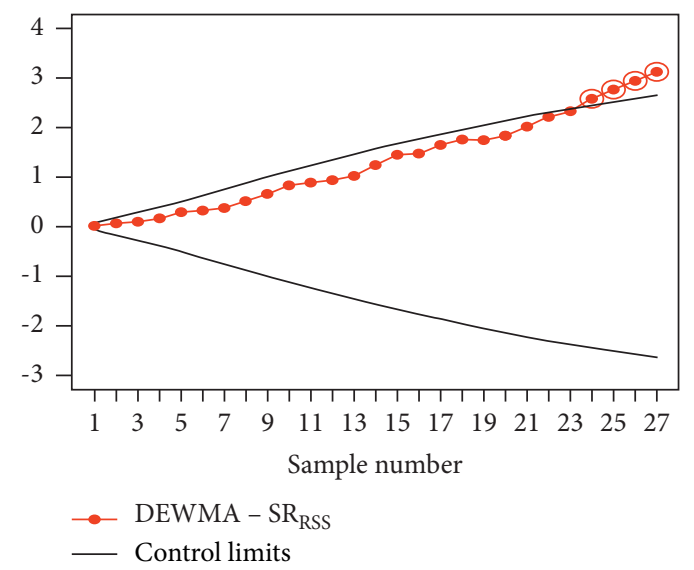

FIgURE 11: Application for the DEWMA - SR RSS control chart when $L=1.578, \lambda=0.05$, and $\mathrm{ARL}_{0}=370$.

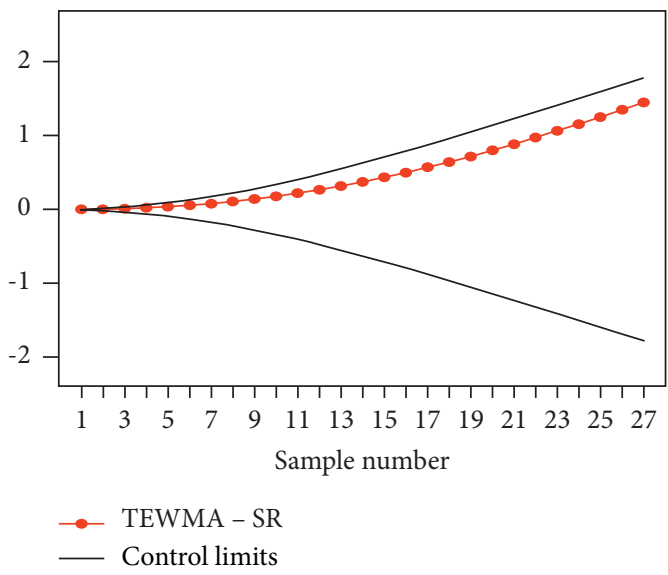

Figure 12: Application for the TEWMA-SR control chart when $L=1.75, \lambda=0.05$, and $\mathrm{ARL}_{0}=370$.

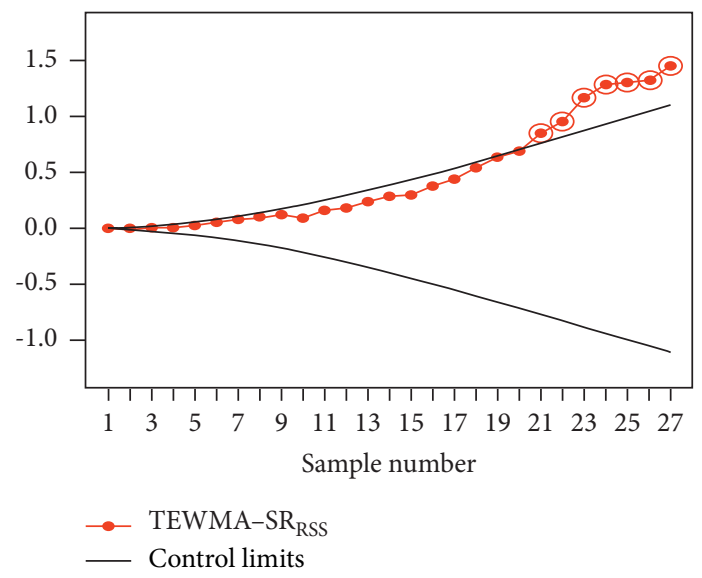

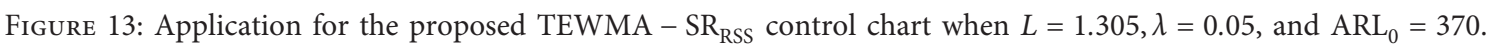

These findings indicate that the proposed TEWMA - SR $\mathrm{RSS}$ has improved performance for the monitoring of the process location as compared to DEWMA - $\mathrm{SR}_{\mathrm{RSS}}$ and TEWMA-SR control charts. Hence, the proposed TEWMA-SR $\mathrm{RSS}$ control chart under RSS has a better ability to detect shifts in process location.

\section{Summary, Conclusions, and Recommendations}

The ranked set sampling (RSS) technique is preferred over the simple random sampling (SRS) for the processes when the estimations are destructive or costly, and the ranking of 
observational data is comparatively simple. Similarly, the nonparametric (NP) control charts are very useful to monitor shifts in the process parameters when the distribution of the underlying process is questionable or unknown. So, this study proposes an NP triple exponentially weighted moving average (TEWMA) Wilcoxon signed-rank (SR) control chart under the RSS technique (represented as TEWMA - $\mathrm{SR}_{\mathrm{RSS}}$ control chart) using different continuous symmetric distributions. The Monte Carlo simulation method is used to obtain the numerical results of the proposed TEWMA - $\mathrm{SR}_{\mathrm{RSS}}$ control chart along with other existing control charts. The performance of TEWMA - $\mathrm{SR}_{\mathrm{RSS}}$ control chart is substantially better than the SRS-based NP TEWMA-SR, TEWMA sign, TEWMA $-\bar{X}$, and double EWMA-SR (DEWMA - SR $\mathrm{RSS}_{\text {) }}$ control charts. A real-life example is also provided to demonstrate how the proposed control chart can be used in practice. Therefore, to adopt a robust and efficient control chart, the proposed control chart gives an alternate choice to quality practitioners. The proposed work can be extended to the multivariate scenario.

\section{Abbreviations}

\begin{tabular}{|c|c|}
\hline ARL: & Average run length \\
\hline $\mathrm{ARL}_{0}:$ & In-control average run length \\
\hline $\mathrm{ARL}_{1}:$ & Out-of-control average run length \\
\hline CUSUM: & Cumulative sum \\
\hline CUSUM-SR: & CUSUM Wilcoxon signed-rank \\
\hline CUSUM-SN: & CUSUM sign \\
\hline $\mathrm{CN}:$ & Contaminated normal \\
\hline DEWMA-SR: & Double EWMA-SR \\
\hline DEWMA: & Double EWMA \\
\hline DEWMA - SR RSS $_{1}$ & Double EWMA-SR with RSS \\
\hline DEWMA $_{\mathrm{TBE}}:$ & $\begin{array}{l}\text { One-sided DEWMA for time between } \\
\text { events }\end{array}$ \\
\hline DEWMA-SR: & Double NP EWMA-SR \\
\hline DEWMA $-\bar{X}$ : & Double EWMA mean \\
\hline DRSS: & Double RSS \\
\hline EWMA: & $\begin{array}{l}\text { Exponentially weighted moving } \\
\text { average }\end{array}$ \\
\hline EWMA - SR RSS $_{1}$ & NP EWMA-SR with RSS \\
\hline $\mathrm{EWMA}_{\mathrm{TBE}}:$ & $\begin{array}{l}\text { One-sided EWMA for time between } \\
\text { events }\end{array}$ \\
\hline EWMA-SR: & EWMA Wilcoxon signed-rank \\
\hline EWMA-SN: & EWMA sign \\
\hline GEWMA-SR: & Generalised EWMA-SR \\
\hline IC: & In-control \\
\hline LOG: & Logistic distribution \\
\hline $\mathrm{LCL}_{\mathrm{EWMA} \mathrm{SR}_{\mathrm{RSS}}}:$ & Lower control limit of EWMA - SR $\mathrm{RSS}$ \\
\hline $\mathrm{LCL}_{\left(\mathrm{TEWMA} \mathrm{SR}_{\mathrm{RSS}}\right)}:$ & $\begin{array}{l}\text { Lower control limit of } \\
\text { TEWMA }-\mathrm{SR}_{\mathrm{RSS}}\end{array}$ \\
\hline $\mathrm{LCL}_{\text {DEWMA-SR RSS }}:$ & $\begin{array}{l}\text { Lower control limit of } \\
\text { DEWMA }-S_{R S S}\end{array}$ \\
\hline MEC: & Mixed EWMA-CUSUM \\
\hline MCE: & Mixed CUSUM-EWMA \\
\hline
\end{tabular}

MxMEC: $\quad$ Auxiliary information based MEC

MxMCE: Auxiliary information based MCE

NP: Nonparametric

OOC: Out-of-control

ODRSS: $\quad$ Order DRSS

OIDRSS: $\quad$ Order imperfect DRSS

RSS: $\quad$ Ranked set sampling

RL: $\quad$ Run length

SDRL: $\quad$ Standard deviation of the run length

SRS: $\quad$ Simple random sampling

SPC: $\quad$ Statistical process control

SR: $\quad$ Wilcoxon signed-rank

TEWMA: Triple TEWMA

TEWMA - $\mathrm{SR}_{\mathrm{RSS}}$ : Triple EWMA with SR under RSS

TEWMA-SR: TEWMA with SR

TEWMA-SN: TEWMA signed-rank test

TEWMA $_{\mathrm{TBE}}$ : One-sided TEWMA for time between

TEWMA $-\bar{X}: \quad$ TEWMA mean

$\mathrm{UCL}_{\text {EWMA-SR }}$ : $\quad$ Upper control limit of EWMA - $\mathrm{SR}_{\mathrm{RSS}}$

$\mathrm{UCL}_{\mathrm{DEWMA}-\mathrm{SR}_{\mathrm{RSS}}}$ : Upper control limit of

DEWMA - SR $\mathrm{RSS}$

$\mathrm{UCL}_{\left(\mathrm{TEWMA}-\mathrm{SR}_{\mathrm{RSS}}\right)}$ : Upper control limit of

Symbols TEWMA - $\mathrm{SR}_{\mathrm{RSS}}$

$\delta: \quad$ Amount of shift in process location

$\sigma: \quad$ Standard deviation

n: $\quad$ Sample size

$\lambda$ : Smoothing constant

$\epsilon: \quad$ Belong to

$t(v)$ : Student's $t$ distribution with degree of freedom $(v)$

$\omega_{0}^{2}$ : Variance

$E_{0}: \quad$ Initial value of EWMA $-\mathrm{SR}_{\mathrm{RSS}}$

L: $\quad$ Control limit coefficient

$E_{q}: \quad$ Plotting statistic of EWMA $-\mathrm{SR}_{\mathrm{RSS}}$

$\mathrm{DE}_{q}$ : Plotting statistic of DEWMA $-\mathrm{SR}_{\mathrm{RSS}}$

$\mathrm{TE}_{q}^{q}$ : Plotting statistic of TWMA $-\mathrm{SR}_{\mathrm{RSS}}$

$\varphi_{0}: \quad$ Median of ranked values

$R_{q j(h)}^{+}$: Rank of absolute deviations

$\mathrm{DE}_{0}$ : Initial value of DEWMA $-\mathrm{SR}_{\mathrm{RSS}}$

$\mathrm{TE}_{0}$ : Initial value of TEWMA $-\mathrm{SR}_{\mathrm{RSS}}$

$m$ : Number of cycles

$r$ : Number of iterations.

\section{Data Availability}

The real-life data used to support the findings of this study are included within the article.

\section{Conflicts of Interest}

The authors declare that they have no conflicts of interest.

\section{Acknowledgments}

The author Saddam Akber Abbasi acknowledges Qatar University for providing excellent research facilities. 


\section{References}

[1] W. A. Shewhart, Economic Control of Quality Manufactured Product, D. Van Nostrand Company, Inc. (reprinted by the American Society for Quality Control in 1980), Milwaukee, WI, USA, 1931.

[2] E. S. Page, "Continuous inspection schemes," Biometrika, vol. 41, pp. 100-115, 1954.

[3] S. W. Roberts, "Control chart tests based on geometric moving averages," Technometrics, vol. 1, no. 3, pp. 239-250, 1959.

[4] V. Alevizakos, K. Chatterjee, and C. Koukouvinos, "Nonparametric triple exponentially weighted moving average signed-rank control chart for monitoring shifts in the process location," Quality and Reliability Engineering International, vol. 37, 2021.

[5] S. T. Bakir and M. R. Reynolds, "A nonparametric procedure for process control based on within-group ranking," Technometrics, vol. 21, no. 2, pp. 175-183, 1979.

[6] R. W. Amin and A. J. Searcy, "A nonparametric exponentially weighted moving average control scheme," Communications in Statistics-Simulation and Computation, vol. 20, no. 4, pp. 1049-1072, 1991.

[7] S. T. Bakir, "A distribution-free Shewhart quality control chart based on signed-ranks," Quality Engineering, vol. 16, no. 4, pp. 613-623, 2004.

[8] N. Balakrishnan, I. S. Triantafyllou, and M. V. Koutras, "Nonparametric control charts based on runs and Wilcoxontype rank-sum statistics," Journal of Statistical Planning and Inference, vol. 139, no. 9, pp. 3177-3192, 2009.

[9] S.-F. Yang and S. W. Cheng, "A new non-parametric CUSUM mean chart," Quality and Reliability Engineering International, vol. 27, no. 7, pp. 867-875, 2010.

[10] S.-F. Yang, J.-S. Lin, and S. W. Cheng, "A new nonparametric EWMA sign control chart," Expert Systems with Applications, vol. 38 , no. 5, pp. 6239-6243, 2011.

[11] M. A. Graham, S. Chakraborti, and S. W. Human, "A nonparametric EWMA sign chart for location based on individual measurements," Quality Engineering, vol. 23, no. 3, pp. 227-241, 2011.

[12] N. Chakraborty, S. Chakraborti, S. W. Human, and N. Balakrishnan, "A generally weighted moving average signed-rank control chart," Quality and Reliability Engineering International, vol. 32, 2016.

[13] M. A. Raza, T. Nawaz, M. Aslam, S. H. Bhatti, and R. A. K. Sherwani, "A new nonparametric double exponentially weighted moving average control chart," Quality and Reliability Engineering International, vol. 36, no. 1, pp. 68-87, 2020.

[14] J.-C. Malela-Majika, "New distribution-free memory-type control charts based on the Wilcoxon rank-sum statistic," Quality Technology and Quantitative Management, vol. 18, no. 2, pp. 135-155, 2021.

[15] Z. He, Y. Gao, L. Qu, and Z. Wang, "A nonparametric CUSUM scheme for monitoring multivariate time-betweenevents-and-amplitude data with application to automobile painting," International Journal of Production Research, pp. 1-18, 2021.

[16] G. A. Mclntyre, "A method for unbiased selective sampling using ranked sets," Australian Journal of Agricultural Research, vol. 3, no. 4, pp. 385-390, 1952.

[17] T. R. Dell and J. L. Clutter, "Ranked set sampling theory with order statistics background," Biometrics, vol. 28, no. 2, pp. 545-555, 1972.
[18] A. Haq, J. Brown, E. Moltchanova, and A. I. Al-Omari, "Partial ranked set sampling design," Environmetrics, vol. 24, no. 3, pp. 201-207, 2013.

[19] R. D. Salazar and A. K. Sinha, "Control chart x based on ranked set sampling," Comunicacion Tecica, vol. 1, no. 97, p. 9, 1997.

[20] H. Muttlak and W. Al-Sabah, "Statistical quality control based on pair and selected ranked set sampling," Pakistan Journal of Statistics, vol. 19, 2003.

[21] M. a. Abujiya and H. Muttlak, "Quality control chart for the mean using double ranked set sampling," Journal of Applied Statistics, vol. 31, no. 10, pp. 1185-1201, 2004.

[22] A. I. Al-Omari and A. Haq, "Improved quality control charts for monitoring the process mean, using double-ranked set sampling methods," Journal of Applied Statistics, vol. 39, no. 4, pp. 745-763, 2012.

[23] A. Haq, J. Brown, and E. Moltchanova, "A new maximum exponentially weighted moving average control chart for monitoring process mean and dispersion," Quality and Reliability Engineering International, vol. 31, no. 8, pp. 15871610, 2014.

[24] A. Haq, J. Brown, E. Moltchanova, and A. I. Al-Omari, "Effect of measurement error on exponentially weighted moving average control charts under ranked set sampling schemes," Journal of Statistical Computation and Simulation, vol. 85, no. 6, pp. 1224-1246, 2015.

[25] A. Haq and M. B. C. Khoo, "A new synthetic control chart for monitoring process mean using auxiliary information," Journal of Statistical Computation and Simulation, vol. 86, no. 15, pp. 3068-3092, 2016.

[26] Z. Abbas, H. Z. Nazir, M. Abid, N. Akhtar, and M. Riaz, "Enhanced nonparametric control charts under simple and ranked set sampling schemes," Transactions of the Institute of Measurement and Control, vol. 42, no. 14, pp. 2744-2759, 2020.

[27] S. E. Shamma and A. K. Shamma, "Development and evaluation of control charts using double exponentially weighted moving averages," International Journal of Quality and Reliability Management, vol. 9, no. 6, pp. 18-25, 1992.

[28] S. M. Anwar, M. Aslam, S. Ahmad, and M. Riaz, "A modifiedmxEWMA location chart for the improved process monitoring using auxiliary information and its application in wood industry," Quality Technology and Quantitative Management, vol. 17 , no. 5, pp. 561-579, 2020.

[29] M. Aslam and S. M. Anwar, "An improved Bayesian Modified-EWMA location chart and its applications in mechanical and sport industry," PLoS One, vol. 15, no. 2, Article ID e0229422, 2020.

[30] S. M. Anwar, M. Aslam, B. Zaman, and M. Riaz, "Mixed memory control chart based on auxiliary information for simultaneously monitoring of process parameters: an application in glass field," Computers and Industrial Engineering, vol. 156, Article ID 107284, 2021.

[31] K. Chatterjee, C. Koukouvinos, and A. Lappa, "A new S2TEWMA control chart for monitoring process dispersion," Quality and Reliability Engineering International, vol. 37, no. 4, pp. 1334-1354, 2020.

[32] V. Alevizakos, K. Chatterjee, and C. Koukouvinos, "A nonparametric triple exponentially weighted moving average sign control chart," Quality and Reliability Engineering International, vol. 37, no. 4, pp. 1504-1523, 2021.

[33] V. Alevizakos, K. Chatterjee, and C. Koukouvinos, "The triple exponentially weighted moving average control chart," 
Quality Technology and Quantitative Management, vol. 18, no. 3, pp. 326-354, 2021.

[34] D. H. Kim and Y. C. Kim, "Wilcoxon signed rank test using ranked-set sample," Korean Journal of Computational and Applied Mathematics, vol. 3, no. 2, pp. 235-243, 1996.

[35] M. Abid, H. Z. Nazir, M. Riaz, and Z. Lin, "Use of ranked set sampling in nonparametric control charts," Journal of the Chinese Institute of Engineers, vol. 39, no. 5, pp. 627-636, 2016.

[36] S. Chakraborti and M. Graham, "Nonparametric (distribution-free) control charts: an updated overview and some results," Quality Engineering, vol. 31, pp. 1-22, 2019.

[37] M. Abid, H. Z. Nazir, M. Riaz, and Z. Lin, "An efficient nonparametric EWMA Wilcoxon signed-rank chart for monitoring location," Quality and Reliability Engineering International, vol. 33, no. 3, pp. 669-685, 2017. 